University of Rhode Island

DigitalCommons@URI

Open Access Dissertations

2019

\title{
A WEARABLE, CONFIGURABLE FUNCTIONAL NEAR-INFRARED SPECTROSCOPY SYSTEM FOR WIRELESS NEUROIMAGING
}

Manob Jyoti Saikia

University of Rhode Island, manob@uri.edu

Follow this and additional works at: https://digitalcommons.uri.edu/oa_diss

\section{Recommended Citation}

Saikia, Manob Jyoti, "A WEARABLE, CONFIGURABLE FUNCTIONAL NEAR-INFRARED SPECTROSCOPY SYSTEM FOR WIRELESS NEUROIMAGING" (2019). Open Access Dissertations. Paper 890.

https://digitalcommons.uri.edu/oa_diss/890

This Dissertation is brought to you for free and open access by DigitalCommons@URI. It has been accepted for inclusion in Open Access Dissertations by an authorized administrator of DigitalCommons@URI. For more information, please contact digitalcommons-group@uri.edu. 
A WEARABLE, CONFIGURABLE FUNCTIONAL NEAR-INFRARED SPECTROSCOPY SYSTEM FOR WIRELESS NEUROIMAGING

BY

MANOB JYOTI SAIKIA

A DISSERTATION SUBMITTED IN PARTIAL FULFILLMENT OF THE REQUIREMENTS FOR THE DEGREE OF

DOCTOR OF PHILOSOPHY

IN

ELECTRICAL ENGINEERING

UNIVERSITY OF RHODE ISLAND

2019 
DOCTOR OF PHILOSOPHY DISSERTATION

$\mathrm{OF}$

MANOB JYOTI SAIKIA

APPROVED:

Dissertation Committee:

$\begin{array}{ll}\text { Major Professor } & \text { Kunal Mankodiya } \\ & \text { Walter G. Besio } \\ & \text { Manbir Sodhi } \\ & \text { Nasser H. Zawia } \\ & \text { DEAN OF THE GRADUATE SCHOOL }\end{array}$

UNIVERSITY OF RHODE ISLAND

2019 


\begin{abstract}
Decoding the human's brain functional architecture is the most profound and far-reaching scientific challenges of our time. Currently existing noninvasive brain imaging technologies are constrained by costly, bulky and fixed hardware that precludes imaging of the functioning brain in a wide range of temporal and naturalistic environments.

This doctoral dissertation focused on designing and developing of a new generation portable, wearable, configurable, and wireless functional near-infrared spectroscopy (fNIRS) neuroimaging system that allows us to monitor and study brain function in a naturalistic environment. The developed optical system maps changes in chromophore concentration, oxy-hemoglobin $\left(\mathrm{HbO}_{2}\right)$ and deoxyhemoglobin $(\mathrm{Hb})$ of the cortical surface of the brain in response to the human brain functions noninvasively.

The fNIRS hardware system was designed based on the Internet-of-Things (IoT) platform using Intel Edison for onboard intelligence, configurability and data transmission. The analog and digital circuits were designed and developed. The fNIRS controlling unit consists of three printed circuit boards (PCBs) sandwiched together: (1) embedded system PCB, (2) analog circuit PCB and (3) digitization PCB. We programmed the system to perform highly complex operations such as montage configuration, sequential NIR light injection and low-intensity backreflected diffused light measurement from the cortical area of the brain at two wavelengths, data conversion, computation, and wireless data transmission, etc. The portable fNIRS system was capable of transferring multi-channel fNIRS data to a computer in real-time. The fNIRS channels are combinations of light sources and detectors.
\end{abstract}

The light-emitting diode (LED) and silicon photodiode (Si-PD) detector based 
fNIRS optodes (source and detector) were developed. We used modern design tools such as 3D printing and laser cutting to fabricate human-centered fNIRS optodes. Feedback was taken from participants of different groups throughout the iterative design process. Two types of fNIRS optodes were designed; one was based on forehead patch and the other was integrable into an electrode head cap that can be placed along with electroencephalography (EEG) electrodes.

Our software architecture wirelessly connects the fNIRS system with a computer or Android tablet through WiFi and interacts to send configuration settings and also to receive fNIRS data in real-time. A host computer connects to the fNIRS control unit via authentication and performs bi-directional communication in real-time to instruct the fNIRS controller to operate in a synchronized manner. The host computer also simultaneously collects and processes the fNIRS signal, and displays hemodynamics responses. A MatLab-based graphical user interface (GUI) software was developed to control the fNIRS system, perform advanced fNIRS data processing, and to visualize multi-channel $\mathrm{HbO}_{2}$ and $\mathrm{Hb}$ in real-time.

The fNIRS system was also capable of synchronizing with the EEG systems to simultaneously collect fNIRS and EEG signal for the multimodal brain imaging. The hardware and software of the fNIRS system were evaluated for its safety, performance and working. After quantitively comparing our fNIRS system with the other portable fNIRS systems proposed by other researchers, we found that our system has several advantages in terms of portability, connectivity, wearability, configurability, comfortability, frame rate, and resolution, etc. for the naturalistic brain imaging.

Experimental studies on human participants in naturalistic environments with different experimental protocols were performed to validate the working of our fNIRS system. These experiments not only verified the working of the fNIRS 
system but also, we studied how the brain's hemodynamic activities are modulated by different tasks, such as meditative breathing exercise, mental arithmetic task, and working memory task, etc.

The first human subject experimental study with our fNIRS system was performed to measure the $\mathrm{HbO}_{2}$ and $\mathrm{Hb}$ concentration changes at the regional muscle (forearm) due to the arterial occlusion. The results of the forearm muscle response during arterial occlusion experiment on the 8 participants proved working of the hardware and software of our fNIRS system.

The second experiment was to image prefrontal cortex of the brain using our fNIRS system on 8 participants while participants performed meditative breathing exercise. The results showed that the system was able to image hemodynamic activities of the brain in this experiment. Also, we derived heart rate variability from the fNIRS data.

In the third experiment, we performed brain imaging while participants were performing mental arithmetic tasks with the difficulty levels of the task in the increasing order to increase the cognitive load. We found that hemodynamic response to the cortical activity rises in response to the mental arithmetic over time. The results also showed that the three stimulus-evoked hemodynamic responses due to the three difficulty levels of the tasks cause three peaks of $\mathrm{HbO}_{2}$ of three different amplitudes.

In the fourth experiment, we performed experimental studies using our fNIRS system to image prefrontal cortex (PFC) areas of the brain of 13 participants while they performed n-back working memory (WM) tasks. We created an n-back experimental protocol that had 16 trials (task blocks) in one run to access four different levels of WM loads and rest periods. Our results showed an incremental brain's hemodynamic activity with the incremental WM load. The results proved 
that our fNIRS system is instrumental in assessing and distinguishing different WM loads. We observed consistent hemodynamic activities over both left and right hemispheres of the brain during all the trials. However, we found slightly higher left PFC activation than the right one.

We also implemented machine learning (ML) method to classify working memory load from the fNIRS signal. We have extracted eight important features from the hemodynamic brain signals and trained six different k-nearest neighbor $(\mathrm{k}-\mathrm{NN})$ ML classifiers. The performance of the six k-NN classifiers was evaluated with the experimental data sets. The results showed that Weighted and Fine k-NN performed best (95\%) in classifying 5 different WM loads.

We also performed multimodal EEG and fNIRS experiments placing tripolar EEG (tEEG) electrodes and fNIRS optodes together at the same time using a common montage cap. The fNIRS measured regional cerebral hemodynamic response complimenting EEG, that directly measures the neural activity of the brain. The optodes' and EEG electrode's actual locations on the scalp during experiments were translated to a standard 10-20 electrode placement system to present fNIRS and EEG channels on a standard brain atlas. The fNIRS and EEG channels were mapped accordingly and topographically presented the brain activities in the multimodal brain studies.

The resulting novel fNIRS system provided an essential tool that is accessible to the broad research and medical community for brain and behavioral research in the naturalistic environments, with extensive applicability in neuroscience, cognitive science, and cognitive psychology. 


\section{ACKNOWLEDGMENTS}

To think that this Ph.D. dissertation is a milestone in my academic career only because of individual effort that would be a grave mistake. I have been truly blessed with the finest group of people who have inspired and guided me throughout my dissertation work and provided assistance for my venture. There are no proper words to convey my deep gratitude to these folks for helping me through this great endeavor.

First and foremost, I would like to express my deepest gratitude to my major professor, Prof. Kunal Mankodiya for his extended great support throughout my Ph.D. study at the University of Rhode Island (URI). I am fortunate, delighted and proud to have worked with him. He had been always a great source of inspiration from his simplicity, friendliness, and encouragement. He was really helpful whenever I approached him and time had never been a constraint for my discussion with him.

I express my sincere gratitude to Prof. Rajan Kanhirodan, Dept. of Physics, Indian Institute of Science (IISc), Bangalore, India, who introduced me to the wonderful area of diffuse optical tomography and its application in medical imaging with sharing his abundance of knowledge patiently, constant support and encouraging guidance. His advice and encouragement were always important guiding lights towards my personal and professional development.

I owe my heartiest gratitude to Prof. Walter G. Besio and Prof. Manbir Sodhi for serving on my doctoral committee. I thank for their encouraging guidance and valuable suggestions during my research work. Without their guidance and support, this research would not have been completed.

I also wish to express my sincere gratitude to the entire faculty members of the

Electrical, Computer, and Biomedical Engineering (ECBE) department of URI for 
providing a cordial, friendly atmosphere and support throughout my stay in URI. The department staffs were also very helpful, especially Lisa Pratt and Meredith Leach Sanders.

I express my sincere thanks to Prof. Paul Bueno de Mesquita, Director of URI Center for Nonviolence and Peace Studies for valuable advice.

I wish to express my thanks to all my colleagues and friends, Dr. Mohammadreza Abtahi, Gozde Cay, Dylan Kennedy, Joanne Hall, Lynn McCane, Tanyya Wang, Dr. Shunan Li, Aras Dar, Indrani Mandal, Nick Constant, Debanjan Borthakur, Brandon Williams, Matt Constant, Andrew Peltier, Arar Alkhader, Scott Selig, Sawyer Nichols, Richard Melpignano, Graem Timmons, Preston Steele, Bahram Borgheai, Sarah Hosni, Roohi Jafari, John McLinden, Enayet Rahman, Alyssa Zisk, Jay Vincelli, Dr. Rosa Ghatee and Joshua V. Gyllinsky.

I would like to acknowledge my sincere thanks to my past colleagues and friends from Indian Institute of Science, Dr. Rakesh Manjappa, Dr. Rasmi C K, Dr. Biswajit Medhi, Dr. Mayanglambam Suheshkumar Singh, Dr. Dibbyan Mazumder, Dr. Jem Teresa, Dr. Samir Kumar Biswas, Dr. Tushar Kanti Bera, Dr. Deepa K G, Dr. Eswaramoorthy Vardharaj, and Dr. Sriram Chandran.

I would like to thank our collaborators, Prof. Sridhar Sunderam and Prof. Guoqiang Yu from the University of Kentucky, and Prof. Lei Ding and Prof. Han Yuan from the University of Oklahoma.

Above all, thank you, all my awesome friends for being in my life. Thank you, Kelsey Sun for standing by me through good times and bad times. Special thanks to Pinki Devi and Carolyn N. Matsumoto for all their support and care.

All this would not have been possible without support from my adorable younger brother, Dr. Sashi Bhusan Saikia and my two elder sisters, who kept up my moral during my downs and supported me. 
Last but not least I express my gratitude to my mother, late Jaya Saikia and my father, Nava Kumar Saikia for their encouragement and blessings. I can not describe how grateful I am to have my mother in my life. Her support, encouragement, sacrifices and unwavering love were undeniably the foundation upon which my life has been built.

The research has been supported by the National Science Foundation (NSF) under Grant No. 1539068. 


\section{DEDICATION}

This dissertation is dedicated to the memories of my mother, Jaya Saikia. 


\section{PREFACE}

This dissertation is organized in the manuscript format consisting of six manuscript chapters as follows:

- Manuscript 1:

M. J. Saikia, and K. Mankodiya, "3D-printed human-centered design of fNIRS optode for the portable neuroimaging", SPIE Photonics West Biomedical Optics (BIOS 2019) Conference, San Francisco, California, United States, February 2-7, 2019. doi: https://doi.org/10.1117/12.2510955

- Manuscript 2:

M. J. Saikia, W. G. Besio and K. Mankodiya, "WearLight: Toward a Wearable, Configurable Functional NIR Spectroscopy System for Noninvasive Neuroimaging," in IEEE Transactions on Biomedical Circuits and Systems, vol. 13, no. 1, pp. 91-102, Feb. 2019. doi: 10.1109/TBCAS.2018.2876089

- Manuscript 3:

M. J. Saikia, G. Cay, J. V. Gyllinsky, and K. Mankodiya,"A configurable wireless optical brain monitor based on internet-of-things services", IEEE International Conference On Electronics, Communication, Computer Technologies and Optimization Techniques (ICEECCOT-2018), Mysuru, India, Dec. 14-15, 2018.

- Manuscript 4:

M. J. Saikia, and K. Mankodiya, "A Wireless fNIRS Patch with Shortchannel Regression to Improve Detection of Hemodynamic Response of Brain", IEEE International Conference On Electronics, Communication, Computer Technologies and Optimization Techniques (ICEECCOT-2018), Mysuru, India, Dec. 14-15, 2018. 
- Manuscript 5:

M. J. Saikia, W. G. Besio and K. Mankodiya, "Mental workload assessment during n-back working memory task using a portable fNIRS system," Submitted to IEEE Transactions on Neural Systems and Rehabilitation Engineering.

- Manuscript 6:

M. J. Saikia, Himanshu Mishra and K. Mankodiya, "A machine learning approach to classify working memory load from optical neuroimaging data," Submitted to IEEE INDICON 2019, Rajkot, India, Dec. 13-15, 2019.

In addition, the author has published other relevant manuscripts. Some of them are from the previous works of the author. All these publications are referenced in this dissertation. The manuscripts are as follows:

- Manuscript 7:

Manob Jyoti Saikia, Kunal mankodiya, and K. Rajan, "A point-of-care handheld Region-of-Interest (ROI) 3D functional Diffuse Optical Tomography (fDOT) system", Optical Tomography and Spectroscopy of Tissue XIII., Vol. 10874. International Society for Optics and Photonics, 2019. doi: $10.1117 / 12.2510926$

- Manuscript 8:

Manob Jyoti Saikia, Rakesh Manjappa, Kunal Mankodiya and K. Rajan, "Depth Sensitivity Improvement of Region-of-Interest Diffuse Optical Tomography from Superficial Signal Regression", Computational Optical Sensing and Imaging, Optical Society of America, 2018. doi: 10.1364/COSI.2018.CM3E.5

- Manuscript 9:

Mohammadreza Abtahi, Gozde Cay, Manob Jyoti Saikia and Kunal 
Mankodiya, "Designing and Testing a Wearable, Wireless fNIRS Patch" 2016 38th Annual International Conference of the IEEE Engineering in Medicine and Biology Society (EMBC), Orlando, USA, August 16-20, 2016. doi: 10.1109/EMBC.2016.7592168

- Manuscript 10:

Manob Jyoti Saikia, and K. Rajan, "Development of handheld near-infrared spectroscopic medical imaging system", OSA Biophotonics Congress 2019: Optics in the Lifesciences Tucson, Arizona, USA, April 15-17, 2019. ISBN: 978-1-943580-54-5

- Manuscript 11:

Manob Jyoti Saikia, and K. Rajan, "A Tabletop Diffuse Optical Tomographic (DOT) Experimental Demonstration System", Optics and Biophotonics in Low-Resource Settings V., Vol. 10869. International Society for Optics and Photonics, 2019. doi: 10.1117/12.2510857

- Manuscript 12:

Manob Jyoti Saikia, Rakesh Manjappa, and K. Rajan, "A Cost-effective LED and Photodetector Based Fast Direct 3D Diffuse Optical Imaging System", European Conference on Biomedical Optics, Optical Society of America, 2017. doi: 10.1117/12.2285940

- Manuscript 13:

Manob Jyoti Saikia and K. Rajan, "Region-of-Interest Diffuse Optical Tomography System", Review of Scientific Instruments, 87, 013701 (2016). doi: http://dx.doi.org/10.1063/1.4939054.

- Manuscript 14:

Manob Jyoti Saikia, K. Rajan and R. M. Vasu, "High-Speed GPU-based 
Fully Three-Dimensional Diffuse Optical Tomographic System", International Journal of Biomedical Imaging, vol. 2014, Article ID 376456, 13 pages. doi: $10.1155 / 2014 / 376456$.

- Manuscript 15:

Manob Jyoti Saikia and Rajan Kanhirodan, "A Cost Effective High Speed Optical Fiber Switch for Diffuse Optical Tomography", Technology Letters, 1(7), 5-9, 2014. ISSN: 2348-8131

- Manuscript 16 :

Manob Jyoti Saikia and K. Rajan, "Development of DOT System For ROI Scanning", 12 $2^{\text {th }}$ International Conference on Fiber Optics and Photonics (Photonics 2014), IIT Kharagpur, India, Dec.13-16, 2014. ISBN: 978-1$55752-882-7$

- Manuscript 17:

Manob Jyoti Saikia and K. Rajan, "High Performance Single and MultiGPU Acceleration for Diffuse Optical Tomography", International Conference on Contemporary Computing and Informatics (IC3I), SJCE, Mysore, India, Nov. 27-29, 2014. doi: 10.1109/IC3I.2014.7019809

- Manuscript 18:

Manob Jyoti Saikia, K. Rajan and R. M. Vasu, "3-D GPU Based Real Time Diffuse Optical Tomographic System", IEEE International Advance Computing Conference (IACC 2014), ITM University, Gurgaon, India, Feb. 21-22, 2014. doi: 10.1109/IAdCC.2014.6779479

- Manuscript 19:

Tushar Kanti Bera, Manobjyoti Saikia, J. Nagaraju, "A Battery-based Constant Current Source (Bb-CCS) for Biomedical Applications", 2013 Inter- 
national Conference on Computing, Communication and Networking Technologies (ICCCNT 2013), Vivekanandha College of Engineering for Women, Tamilnadu, India, July 4-6, 2013. doi: 10.1109/ICCCNT.2013.6726810

The author has also published other relevant conference abstracts as follows:

- Abstract 1:

Manob Jyoti Saikia, Mohammadreza Abtahi and Kunal Mankodiya, "Development of a Wireless Wearable fNIRS System", fNIRS 2016 Conference, Paris, France, Oct. 13-16, 2016.

- Abstract 2:

Manob Jyoti Saikia, R. Manjappa and K. Rajan, "Region-of-Interest Functional Diffuse Optical Tomography System", SPIE Translational Biophotonics 2016, Rice Univ., Houston, Texas, United States, May 16-17, 2016.

- Abstract 3:

Mohammadreza Abtahi, Yalda Shahriari, Manob Jyoti Saikia, Gozde Cay, and Kunal Mankodiya, "EEG-fNIRS Combined Neuroimaging Study on PD patients performing UPDRS Motor Tasks, '"Neurology, P5.8-020", April 9, 2019.

- Abstract 4:

Mohammadreza Abtahi, Amir Mohammad Amiri, Dennis Byrd, Manob Jyoti Saikia and Kunal Mankodiya, "Hand Flipping Detection in fNIRS data using Support Vector Machine", fNIRS 2016 Conference, Paris, France, October 13-16, 2016. 


\section{TABLE OF CONTENTS}

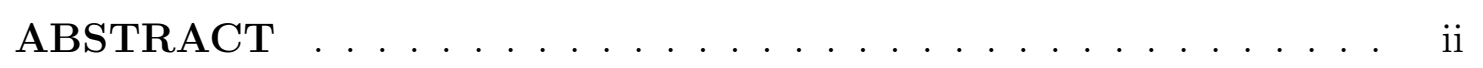

ACKNOWLEDGMENTS .................. vi

DEDICATION .................... ix

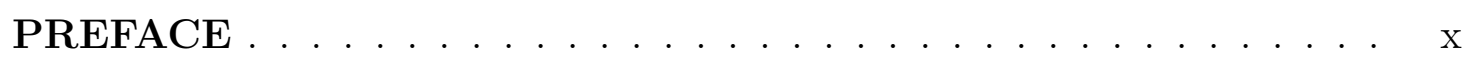

TABLE OF CONTENTS $\ldots \ldots \ldots \ldots \ldots \ldots \ldots$

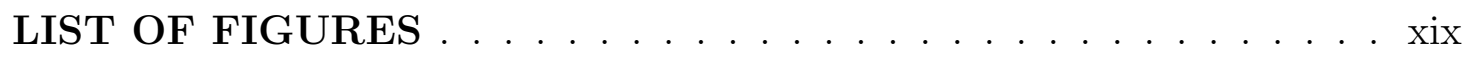

LIST OF TABLES $\ldots \ldots \ldots \ldots \ldots \ldots \ldots \ldots \ldots \ldots \ldots \ldots$ xxiv

\section{MANUSCRIPT}

1 3D-printed human-centered design of fNIRS optode for portable neuroimaging . . . . . . . . . . . . 1

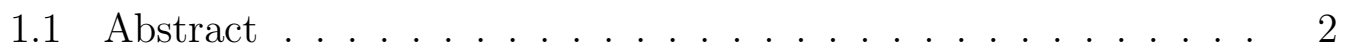

1.2 Introduction . . . . . . . . . . . . . . . . 2

1.3 Methods . . . . . . . . . . . . . . . . . . . 5

1.3.1 Human-centered 3D-printed fNIRS Optode . . . . . 7

1.4 Experimental Studies . . . . . . . . . . . . . . . . . . . . . . . 9

1.4.1 User Experience and Signal Quality . . . . . . . . . . 10

1.5 Result . . . . . . . . . . . . . . . . 11

1.6 Conclusion . . . . . . . . . . . . . . . . . 11

List of References . . . . . . . . . . . . . . . . . . 12

2 WearLight: Towards a Wearable, Configurable Functional NIR Spectroscopy System for Noninvasive Neuroimaging . . 15 


\section{Page}

2.1 Abstract ............................. 16

2.2 Introduction . . . . . . . . . . . . . . . . 16

2.3 Background and State-of-the-Art . . . . . . . . . . . . 20

2.3.1 fNIRS Principle . . . . . . . . . . . . . 20

2.3.2 State-of-the-Art: fNIRS System Design . . . . . . . . . 22

2.4 WearLight fNIRS System Design . . . . . . . . . . . . . 24

2.4.1 fNIRS Controlling Unit ............ 26

2.4.2 Optodes (NIR Light Source and Detector) . . . . . . . 29

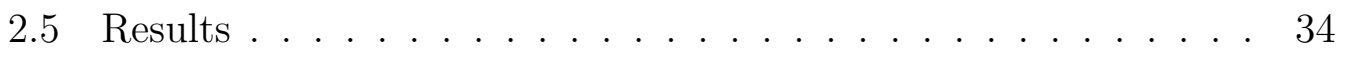

2.5.1 Experiment 1: Arterial Occlusion Experiment .... . 34

2.5.2 Experiment 2: Prefrontal Cortex fNIRS Experiment . . . 36

2.6 Discussion ...................... . . . . 40

List of References . . . . . . . . . . . . . . . . . . . 43

3 A configurable wireless optical brain monitor based on internet-of-things services . . . . . . . . . . . . . 52

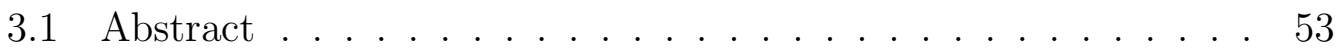

3.2 Introduction . . . . . . . . . . . . . . 54

3.3 Background of fNIRS . . . . . . . . . . . . . . . 57

3.3.1 Related Works . . . . . . . . . . . . . 58

3.4 A Configurable fNIRS System . . . . . . . . . . . . . 59

3.4.1 fNIRS Hardware . . . . . . . . . . . . . . . . 59

3.4.2 Service oriented architecture on embedded board . . . . . 61

3.4.3 Controlling Graphical User Interface (GUI) . . . . . . . . 63

3.5 Results and Discussion . . . . . . . . . . . . . . 66 


\section{Page}

3.5.1 fNIRS Hardware Evaluation . . . . . . . . . . 66

3.5.2 Wireless Communication Testing . . . . . . . . 67

3.5.3 System Performance Evaluation . . . . . . . . . . 67

3.6 Conclusion and Future Work . . . . . . . . . . . . . . . 68

List of References . . . . . . . . . . . . . . . . . . . . 69

4 A wireless fNIRS Patch with short-channel regression to improve detection of hemodynamic response of brain . . . . . . 74

4.1 Abstract ........................... 75

4.2 Introduction . . . . . . . . . . . . . . . . 75

4.3 Background of fNIRS Imaging . . . . . . . . . . . . . 78

4.4 Wireless Wearable fNIRS Patch with a Short-channel . . . . . . 80

4.4.1 fNIRS Optode Patch Design . . . . . . . . . . . 81

4.4.2 fNIRS Controlling Hardware . . . . . . . . . . . . . . 81

4.5 Experimental Studies ................. 84

4.5.1 Hardware and Software Setup . . . . . . . . . . 84

4.5.2 Experimental Protocol ............. 85

4.6 Results and Discussion . . . . . . . . . . . . . . 86

4.7 Conclusion and Future Work . . . . . . . . . . . . . . . . 87

List of References . . . . . . . . . . . . . . . . . . . . . 88

5 Mental workload assessment during n-back working memory task using a portable fNIRS system . . . . . . . . . . . . . 92

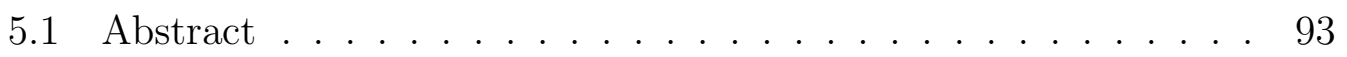

5.2 Introduction . . . . . . . . . . . . . . . . 93

5.3 Materials and Methods . . . . . . . . . . . . . . . . 98 


\section{Page}

5.3.1 Functional Near-infrared Spectroscopy (fNIRS) . . . . . . 98

5.3.2 N-back memory task . . . . . . . . . . . . . . 102

5.3 .3 Experimental setup . . . . . . . . . . . . . . . . . 103

5.3.4 Experimental paradigm . . . . . . . . . . . . . . . 104

5.3 .5 Participants . . . . . . . . . . . . . . . 106

5.3 .6 Data analysis . . . . . . . . . . . . . . . 106

5.4 Results . . . . . . . . . . . . . . . . . . . 107

5.4 .1 Task performance . . . . . . . . . . . . . 107

5.4.2 Brain hemodynamic response . . . . . . . . . . . 108

5.5 Discussion and Conclusion . . . . . . . . . . . . 110

List of References . . . . . . . . . . . . . . . . . . . . . 111

6 A machine learning approach to classify working memory load from optical neuroimaging data $\ldots \ldots \ldots 116$

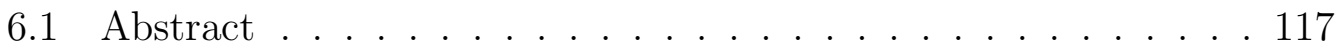

6.2 Introduction . . . . . . . . . . . . . . . . . 117

6.3 Background of fNIRS-based BCI . . . . . . . . . . . . 119

6.4 Materials and methods . . . . . . . . . . . . . . 120

6.4.1 Experimental study . . . . . . . . . . . . . . 120

6.4.2 Machine learning implementation . . . . . . . . . . . 121

6.5 Results and Discussion . . . . . . . . . . . . . . . 124

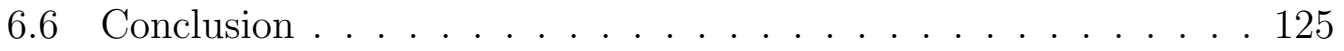

List of References . . . . . . . . . . . . . . 125 


\section{LIST OF FIGURES}

Figure

Page

1 A conceptual diagram of fNIRS headgear and fNIRS system. . . 4

2 fNIRS patch, (a) using flexible 3D printing and (b) laser cutting. 6

3 fNIRS optode design 1 using OPT101 SiPD and dual wavelengths LED. (a) 3D printing, (c) source and detector optode and (c) optode placement. . . . . . . . . . . . . 7

4 fNIRS optode design 2 using ODA-6WB-500M SiPD and dual wavelengths LED. (a) source and detector optode and (b) optode placement. . . . . . . . . . . . . . . 8

$5 \quad$ Electrical testing of the optode pair through pulse oximetry experiment. . . . . . . . . . . . . . . 9 9

$6 \quad$ WearLight fNIRS brain imaging system. A participant wearing optodes on the head using an electrode cap to image prefrontal cortex. . . . . . . . . . . . . . 17

$7 \quad$ The basic principle of fNIRS. (a) fNIRS montage cap containing NIR light source and detector arrangements, and (b) propagation of NIR light through different tissue layers of a human head from a source to a detector. . . . . . . . . . . . . . . 20

8 The plot of absorption spectra of oxy-hemoglobin $\left(\mathrm{HbO}_{2}\right)$ and deoxy-hemoglobin $(\mathrm{Hb})$ of tissue, represented in terms of molar absorption coefficients. Experimental data were taken from OMLC [24] . The wavelengths of the LEDs used for our fNIRS system are indicated by the vertical dashed line. . . . . . . . . . 22

9 A simplified block diagram showing the hardware components of the WearLight fNIRS system. A single WearLight module supports eight dual wavelength LEDs and sixteen detectors. . . 24

$103 \mathrm{D}$ printed optode design. Optodes made of multi-wavelength LEDs (MTMD7885NN24) and Si-PD detectors (ODA-6WB$500 \mathrm{M}$ detectors). . . . . . . . . . . . . 
11 (a) Experimental set-up for arterial occlusion experiment, a participant is wearing a pair of optodes and sphygmomanometer cuff, and (b) forearm muscle response during arterial occlusion experiment. $\Delta H b O_{p 1-p 8}, \Delta H b_{p 1-p 8}$ and $\Delta H b T_{p 1-p 8}$ are the results from the participant no. 1 to participant no. 8 respectively and $\Delta H b O_{\text {mean }}, \Delta H b_{\text {mean }}$ and $\Delta H b T_{\text {mean }}$ are the mean response in the experiment. . . . . . . . . . . . . . . . 35

12 An experimental raw fNIRS data channel during breathing experiment, collected from a participant. . . . . . . . . . . 37

13 Hemodynamic response derived from the experimental fNIRS data channel shown in the Figure 12. . . . . . . . . . . 38

14 Topographic map of $\mathrm{HbO}_{2}$ and $\mathrm{Hb}$ concentration changes on a $3 \mathrm{D}$ cortical surface of the brain at different important time frames from the experimental fNIRS data collected from a participant during the breathing exercise using our WearLight fNIRS system. . . . . . . . . . . . . . . . 39

15 (a) NIR light propagation from the sources to detectors carrying information of the hemodynamic activities of the brain, (b) plot of absorption spectra of $\mathrm{HbO}_{2}$ and $\mathrm{Hb}$ in the NIR window . . . 55

16 Principles of Modified Beer-Lambert Law. . . . . . . . . . . . 56

17 A conceptual diagram of configurable fNIRS system. . . . . . . 58

18 Timing sequence for triggering LEDs. . . . . . . . . . . . . . . 60

19 Different regions of the brain and possible optode montages. . . 62

20 The flowchart of fNIRS application. . . . . . . . . . . . 63

21 fNIRS hardware testing. (a) photodetector signal on digital oscilloscope, (b) testing of fNIRS hardware components. . . . . . 64

22 Data communication between fNIRS hardware and graphical user interface (GUI) testing. GUI displays montages and plots channel data in real-time. . . . . . . . . . . . . . . 65

23 Experimental setup for the arterial occlusion test. . . . . . . . 66 
24 The fNIRS GUI displaying montages and plotting the hemodynamic response. . . . . . . . . . . . . . . . . 68

25 NIR light propagation from sources to detectors carrying information of the hemodynamic activities of the brain. . . . . . . 76

26 Plot of absorption spectra of $\mathrm{HbO}_{2}$ and $\mathrm{Hb}$ in the NIR window. Experimental data were taken from OMLC [23]. . . . . . . . 77

27 a) Principle of Modified Beer-Lambert Law, and (b) sketch of NIR light propagation from a source to a detector through different tissue layers of a brain. Source and detector separating distance $(d)$ is related to optical path length $(L)$ by $L=D P F . d . \quad 79$

28 (a) A conceptual diagram of fNIRS patch connected to a wireless fNIRS controller. (b) The patch has one dual-wavelength LED and three detectors forming two target fNIRS channels (Ch1 and Ch2) and a short-channel (SC). . . . . . . . . . 80

29 Timing sequences for triggering LED (wavelengths $770 \mathrm{~nm}$ and $850 \mathrm{~nm}$ ) and three detector readings (Det 1 - Det 3). . . . . . 82

30 (a) Participant wearing the fNIRS patch, (b) fNIRS hardware testing setup, and (c) fNIRS patch. . . . . . . . . 83

31 GUI software to control the fNIRS system. . . . . . . . . . . 84

32 Oxyhemoglobin concentration changes. (a) and (b) are the fNIRS channels Ch1 and Ch2 using detector 2 and detector 3 reading alone respectively. Red blocks are the stimulation epochs. . . . . . . . . . . . . . . . 85

33 Oxyhemoglobin concentration changes of short-channel using detector 1. Red blocks are the stimulation epochs. . . . . . . . 86

34 Corrected oxyhemoglobin trends using short-channel data regression. (a) and (b) are the corrected fNIRS channels Ch1 and Ch2. Red blocks are the stimulation epochs. . . . . . . . .

35 (a) WearLight fNIRS system, the system consists of an optode cap and acontrol box connected to a battery and (b) participant wearing the fNIRS montage cap having four source and eight detector optodes designed for prefrontal cortex imaging in our n-back WM task. . . . . . . . . . . . . . . . . 
36 Sketch of NIR light propagation from a source to a detector through different tissue layers of a brain forming an fNIRS channel. Source and detector separating distance $(d)$ is related to

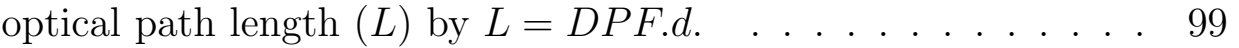

37 Absorption spectra of oxy-hemoglobin $\left(\mathrm{HbO}_{2}\right)$ and deoxyhemoglobin $(\mathrm{Hb})$ of tissue, represented in terms of molar absorption coefficients. Data were taken from OMLC [33]. The wavelengths of the LEDs used for our fNIRS system are indicated by the vertical dashed line. . . . . . . . . . . . . . . 100

38 The fNIRS montage used in the experiment. (a) LEDs (red), detectors (blue) and the fNIRS channels (green line) location on a standard 10-20 EEG system. (b) fNIRS channels (yellow label) onto the head model showing the coverage on the prefrontal areas. Figures are generated using nirsLAB software. . . . . . . 101

39 Experimental paradigm. (a) concept of 0, 1, 2, and 3-back tasks, (b) a task block and each task block has 0, 1, 2 and 3-back conditions and (c) four task blocks each with four n-back pseudorandomized conditions. A complete run takes about 738 seconds. . . . . . . . . . . . . . . . . . 103

40 Increasing oxyhemoglobin $\left(\mathrm{HbO}_{2}\right)$ responses with difficulty levels in the n-back tasks, (a) 0-back, (b) 1-back, (c) 2-back and (d) 3 -back . . . . . . . . . . . . . . . . . . . 107

41 Oxyhemoglobin mean response over the entire participants group and in trials. Rows are the 14 fNIRS channels and column are the 16 n-back trials. The channel number are the same as in the Fig. 38. . . . . . . . . . . . . . . . . . . . . . . . . . . . . 108

42 De-oxyhemoglobin mean response over the entire participants group and in trials. Rows are the 14 fNIRS channels and column are the 16 n-back trials. The channel number are the same as in the Fig. 38. . . . . . . . . . . . . . . . . . . . . . . . . . 109

43 Typical trend of $\mathrm{HbO}_{2}$ (red curve) and $\mathrm{Hb}$ (black curve) due to cortical activation in fNIRS imaging. . . . . . . . . . . 118 
44 (a) Experimental protocol with four n-back conditions (0-back, 1-back, 2-back and 3-back) and rest (R) periods. The 16 random task blocks in one run takes about 720 seconds. (b) fNIRS light source (red) and detector (green) locations on the prefrontal lobe of the brain. . . . . . . . . . . . . . . . . . . . 121

45 Flow chart of our ML implementation. The extracted feature matrix (X) from the fNIRS signal (mean of best three channels) was combined with output matrix $(Y)$ to form $\mathrm{X} 1$ matrix to train the classifier. The trained classifier was tested with input data sets to predict mental states (5 classes). . . . . . . . . . 121

46 Computation time in training the six k-NN classifiers. . . . . . . 124 


\section{LIST OF TABLES}

Table $\quad$ Page

1 Survey Rating Responses . . . . . . . . . . . . . . . . 11

2 Total hemoglobin concentration change $(\Delta H b T)$ in the forearm occlusion experiment (Exp. 1), and $\triangle H b T$ and BPM changes in breathing experiment (Exp. 2) for the eight subjects. . . . . 40

3 Characteristics of portable fNIRS technologies. . . . . . . . . 41

4 Trained six k-NN classifiers and corresponding accuracy in classification . . . . . . . . . . . . . . . . 123 


\section{MANUSCRIPT 1}

3D-printed human-centered design of fNIRS optode for portable neuroimaging

by

Manob Jyoti Saikia, and Kunal Mankodiya

published in

SPIE Photonics West Biomedical Optics (BIOS 2019) Conference, San Francisco, California, United States, February 2-7, 2019. 


\section{$1.1 \quad$ Abstract}

Functional Near-infrared Spectroscopy (fNIRS) is an optical brain imaging technology based on mapping blood oxygenation levels on the cortical surface of the brain. fNIRS has the potential to become a point-of-care brain monitoring system for localized brain measurements in various medical conditions including brain injury and concussion. Although fNIRS electronic circuit has been miniaturized significantly, one of the least elucidated elements of the portable fNIRS systems is the process for developing optodes (light sources and detectors) for the improvements in skin-optode coupling, signal-to-noise ratio (SNR), user's comfortlevel and motion artifact reduction. We have used modern design tools such as 3D printing and laser cutting to fabricate human-centered fNIRS optodes. Feedback was taken from participants of different groups throughout the iterative design process of data collection. Two types of fNIRS optodes were designed; one was based on forehead patch and the other was integrable into an electrode head cap. The optodes were connected to our developed portable fNIRS hardware. The noise characteristics of the optodes for the long-term brain imaging settings, while subjects were performing physical activities, was systemically studied in each of the iterations and designs. The final front-end hardware design was tested on eight subjects with varying head size, shape and skin tone. Experimental results of SNR and resistance to the motion artifacts show that 3D printing can be effective for the development of fNIRS optode. These fNIRS optodes prove to not only be easy to use and comfortable but also capable of acquiring the fNIRS signal with other brain monitoring modalities such as electroencephalography (EEG).

\subsection{Introduction}

Non-invasive neuroimaging technologies are becoming increasingly prevalent due to their bountiful clinical and research applications. These methods improve 
our collective understanding of the brain, leading to more effective treatments and therapies for neurological and psychiatric disorders. The most widely investigated of these technologies are electroencephalography (EEG), and functional magnetic resonance imaging (fMRI). EEG utilizes conductive electrodes placed on the scalp to record the general electrical activity of millions of neurons. However, it is not able to detect this activity at great depth and suffers from low spatial resolution. fMRI has a greater capacity for spatial resolution but has a limited temporal resolution of 0.5 seconds $[1,2]$. Perhaps the greatest limitation of these technologies is the need for immobilization of the subject whilst recording. Thus there is a need for a brain imaging technology with mobile capability. One such technology is entitled functional near-infrared spectroscopy (fNIRS) [3, 4, 5]. By recording changes in the light absorption of brain tissue, fNIRS is able to detect changes in the concentration of oxyhemoglobin and de-oxyhemoglobin due to neural activity $[6]$.

The most popular technique for achieving this is called continuous wave (cw) fNIRS which utilizes constant amplitude NIR light sources in the wavelength range 650-1000 nm and light detectors to probe the brain. In the NIR window overall light absorption in the biological tissue is relatively less $[7,8,9]$. Hence, NIR light can probe tissues significantly deeper. The incident NIR light is scattered and absorbed throughout the brain tissue. The incident NIR light propagates through tissue in a diffusive manner $[10,11,12]$. The back-reflected light from the cortical area of the brain is measured by detectors on the scalp[10, 13, 14]. The light source and detector separating distance greatly influences the probing depth and signal quality $[13,15,3,16,17,18]$. A $2-4 \mathrm{~cm}$ separation is suitable for cw-fNIRS imaging. The light source and detector are commonly known as optodes. From the spectroscopic measurement data, the changes in hemoglobin concentration in 


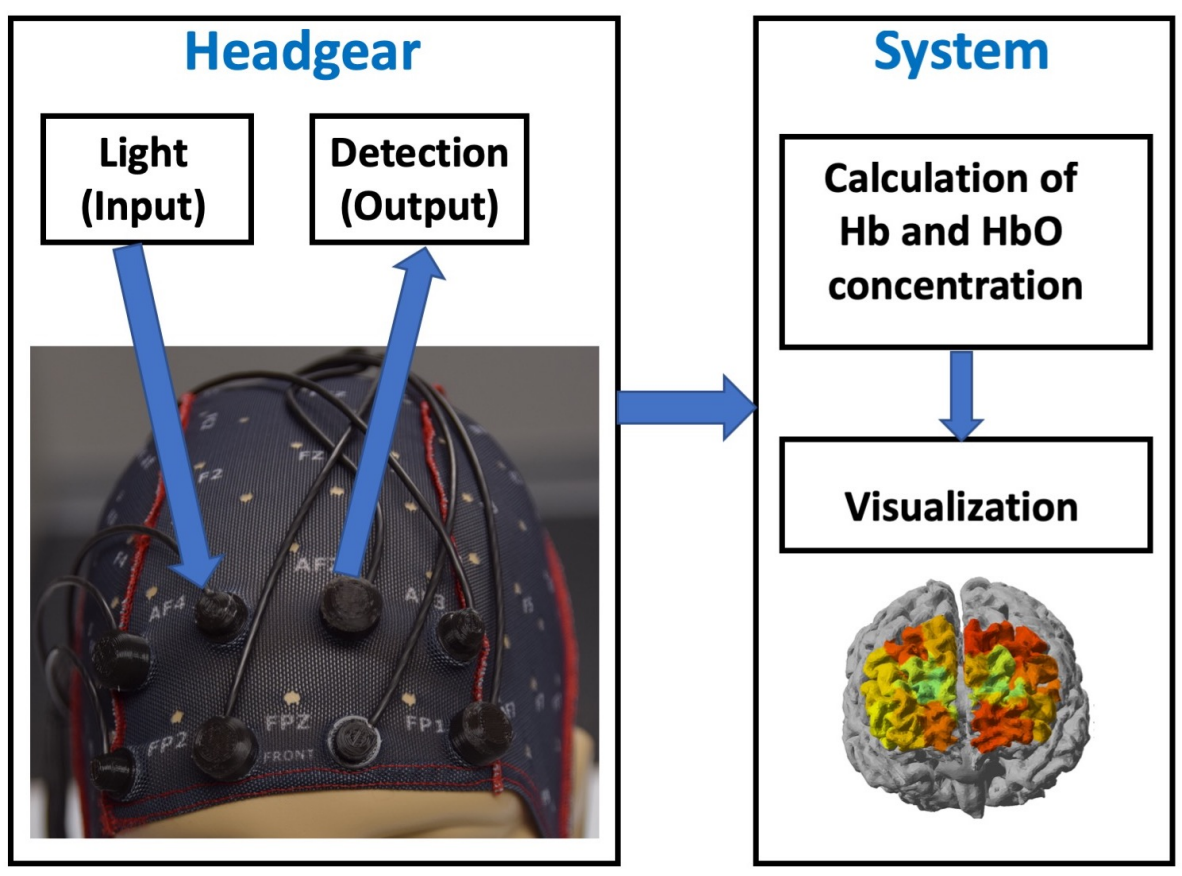

Figure 1. A conceptual diagram of fNIRS headgear and fNIRS system.

the brain are computed using the differential modified Beer-Lambert law [6, 7]. A conceptual diagram of this process is shown in Figure 1 where optodes are placed on the scalp using an electrode cap (headgear) and the measurements are carried out using the fNIRS control system.

Three-dimensional printing (3D printing) and laser cutting are the tools which can be useful for the development of biosensors, however, its applications to fNIRS have yet to be fully explored. There are currently exist a substantial body of work describing the creation of fNIRS systems. One of the least elucidated elements of these systems is the process for developing the optodes for the better coupling to the patient's scalp. The overall signal quality of the fNIRS imaging largely depends on this factor, especially when brain imaging is performed in an unconstrained setting and subjects are allowed to move.

In this paper, we discuss the human-centered 3D printed fNIRS headgear for use in portable neuroimaging. We also explored the design of a wearable fNIRS 
head band using 3D printing and laser cutting technology. We have used multiwavelength LED and silicon photodiode (SiPD) as a light source and detector respectively to design the optodes. We have utilized an iterative process for the design of our 3D printed optode and fNIRS headgear. In the iterative design process, feedback from the subjects of different groups throughout the design process was taken to improve the user comfort and signal quality. Also, noise characteristics were studied in each stage for the applicability of the optodes when the subjects perform physical activities. In order to attach these optodes to a commonplace flexible electrode cap, 3D printed optode holders were designed in the final design. The holder encapsulates a PCB containing electronic components and the LED or SiPD. The final design provides the benefit of integration of the fNIRS optodes with the EEG electrodes. The front-end hardware design was integrated with our laboratory developed portable fNIRS system [5] to test for its human-centered applicability on 8 subjects with varying head sizes. In addition to being comfortable to wear over a long duration of time, it was easy to operate the optodes for a wide range of participants.

\subsection{Methods}

There are currently exist a few different forms of portable fNIRS [19, 20, 21]. One of the greatest impediments to the development of these systems is the lack of a framework for developing the optodes used in the emission and detection of light on the patient's scalp. This paper proposes front-end hardware for an fNIRS system that utilizes a framework, based on the principles of human-centered design to maximize its efficacy, applicability, the quality of the user experience and signal quality.

Human-centered design is a method of innovation employed for towards the goal of increasing the usability of tools and technology by focusing on applied 

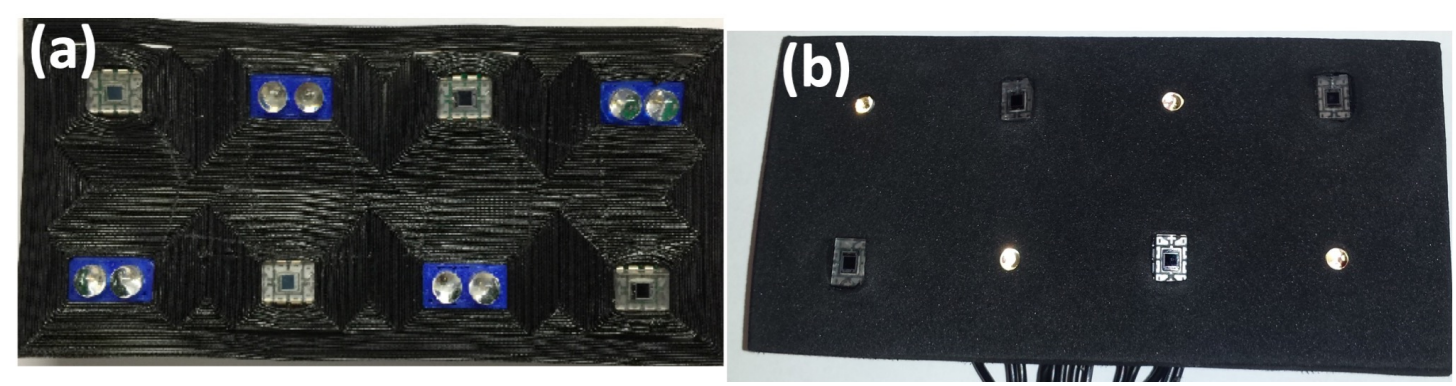

Figure 2. fNIRS patch, (a) using flexible 3D printing and (b) laser cutting.

human factors. The technology being developed can support the intended user's knowledge, attitudes, and behaviors [22]. In this way, the technology is designed to adapt to the user, rather than asking the user to adapt to the technology. Thus, the technology is not only more favorable but also more widely applicable to a broad set of users. Based on the principles of human-centered design, such adaptability is crucial. Human-centered design is best used with an iterative process. At each stage, the user can be consulted so as to maximize the usability of the final iteration. 3D printing and laser cutting are excellent tools for pursuing such a process. 3D printing is a method of creating objects by the conjoining of various materials such as plastic, metals, or powders into a given shape based on a computer-aided design (CAD) [23]. It is advantageous due to its capability for ease of use, cost efficiency, and inclination for rapid and effective prototyping. It has become common for many engineering research laboratories and industries to utilize on-site 3D printers to develop and even manufacture their technologies rather than outsource them. One such application is in pre-surgery neurological modeling [24]. Another is for use in creating scaffolds for bone tissue engineering [25]. Due to 3D printing's capability for rapid prototyping, it is the perfect process for implementing human-centered design principles in our optode design. We have utilized computer-aided design and 3D printing methods for the conceptualization and construction of the framework of the optode discussed in this paper. 
(a)
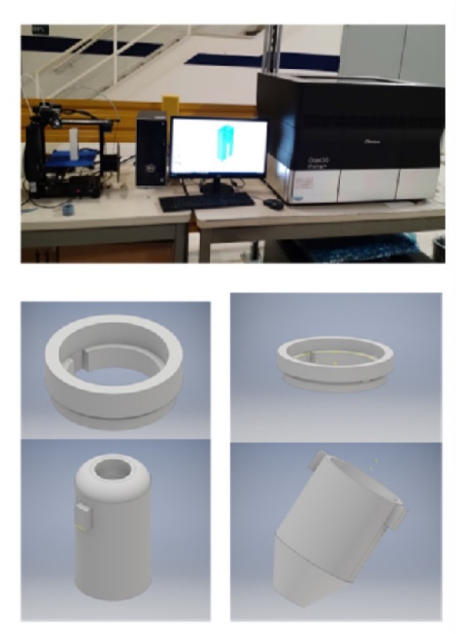

(b)

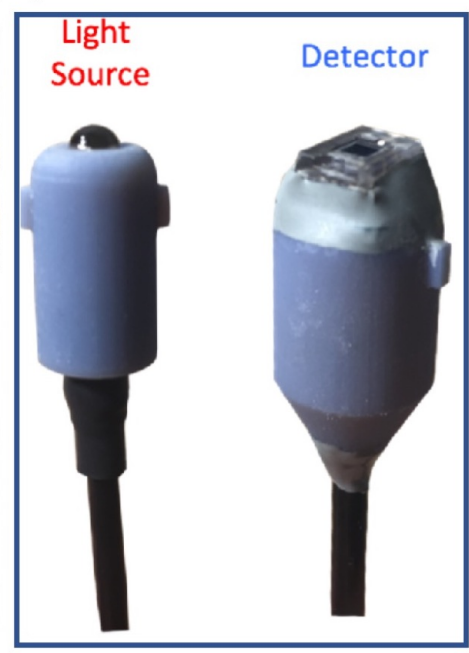

(c)

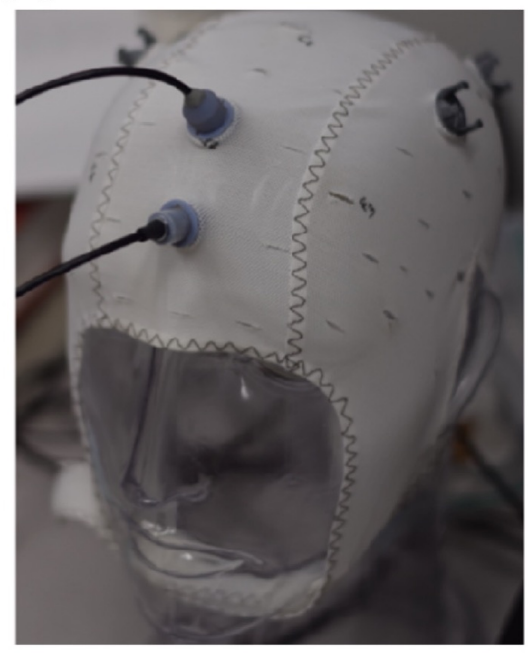

Figure 3. fNIRS optode design 1 using OPT101 SiPD and dual wavelengths LED. (a) 3D printing, (c) source and detector optode and (c) optode placement.

\subsubsection{Human-centered 3D-printed fNIRS Optode}

The first step to designing an optode was to determine a way to affix it to an individual's scalp for the recording of the fNIRS signal over time. The fNIRS patch as shown in Fig. 2 is suitable for imaging of prefrontal cortex of the brain. Fig. 2 (a) is the designed patch using flexible 3D printing and Fig. 2 (b) is the patch using laser cutting technology. In our later design, an electrode cap was decided as the most effective way to place optodes on scalp due to its comfort and utilization in similar in technologies. Additionally, it provided a desirable level of comfort for the user. In order to create detector optode, some form of photodiode must be utilized. The first of these components that were chosen was the OPT101 photodiode with an operational amplifier. Next, a method of coupling the OPT101 with the cap had to be ideated. Due to rapid prototyping capability, a 3D printed holder made of poly-lactic acid (PLA) was designed. The first draft of the said holder was designed as a two parts system. As the OPT101 is a 6pin device, a printed circuit board (PCB) configuration was designed such that it 
(a)

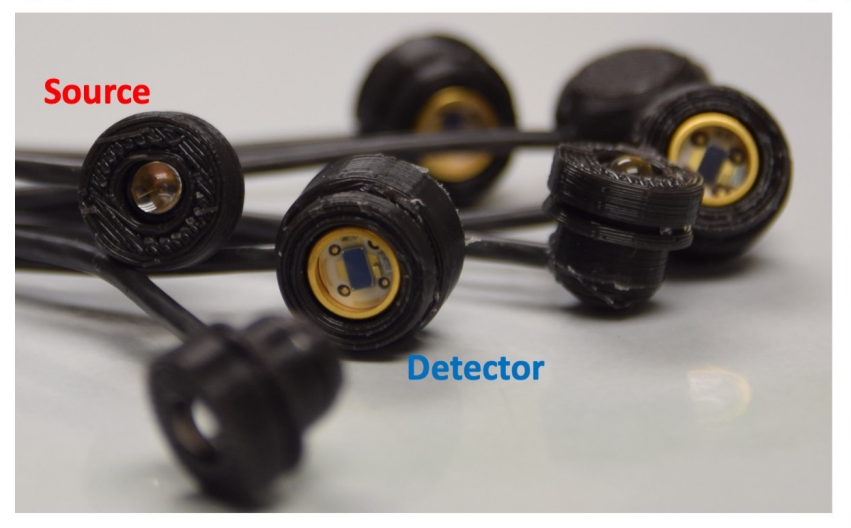

(b)

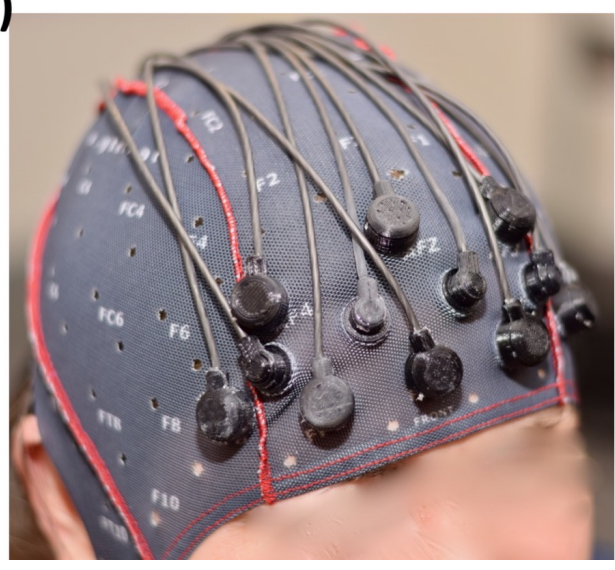

Figure 4. fNIRS optode design 2 using ODA-6WB-500M SiPD and dual wavelengths LED. (a) source and detector optode and (b) optode placement.

could be mounted on a holder. The first of the two holder parts were attached to the OPT101 via said PCB. The second was embedded in the wearable cap. In this way, one could attach and detach the optode conveniently. Concurrently, a PCB and holder were created for the multi-wavelength LED (770nm and $850 \mathrm{~nm}$ ) that is used to emit the light to be detected by the detector optode. Each holder followed the same two-component design. The original CAD holders that were 3D printed are shown in Fig. 3 (a). Fig. 3 (b) and (c) show the fabricated optodes and the optode placement using a cap respectively.

However, effective design is an iterative process and there were errors present in the first design. The resolution of the 3D printers being used was insufficient at ensuring an even coupling between the two holder parts. Additionally, the OPT101 proved relatively less sensitive and did not fit well with the circular nature of the holder. As such, the original design had to be abandoned. A new design had to be created that differed in capability and structure from the former. First, a new photodiode was chosen, the ODA-6WB-500M photodiode because of its higher sensitivity in detecting light. Additionally, its circular design allowed for a better fit within the electrode cap. In order to account for the shortcomings of the 3D 


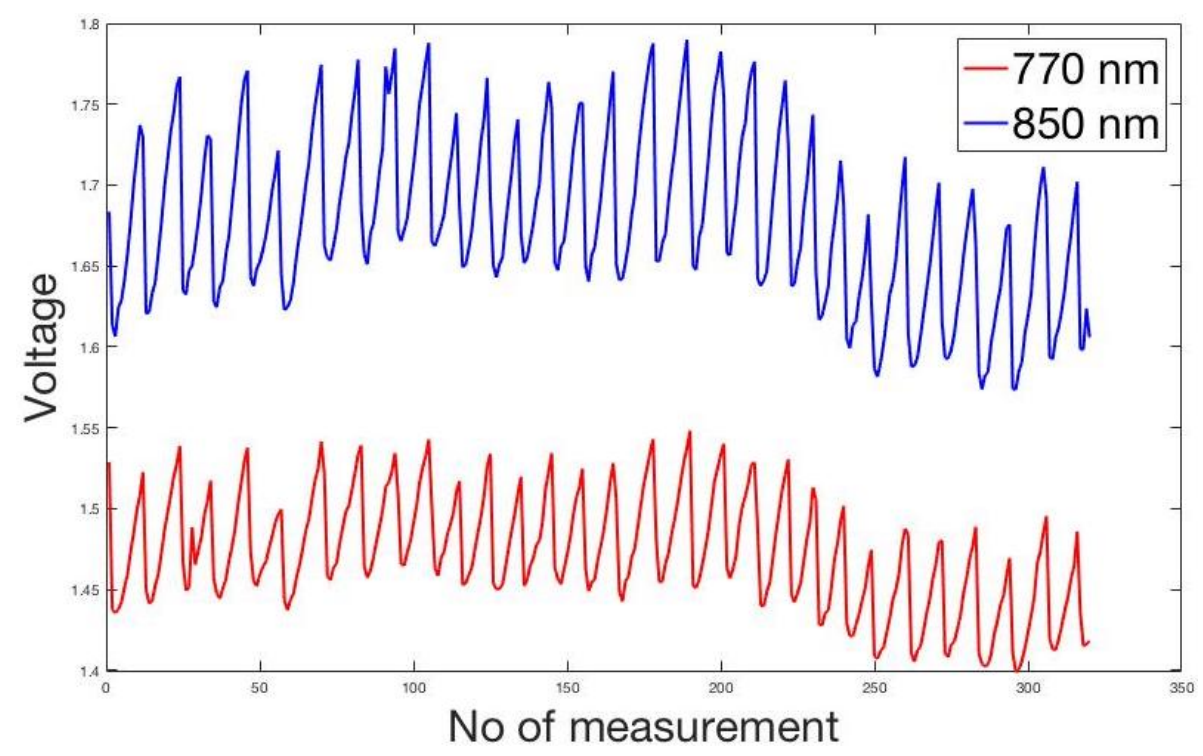

Figure 5. Electrical testing of the optode pair through pulse oximetry experiment.

printer, the two-part design was merged into a one part design with a lid. The top portion consisted of a holding portion and the bottom portion contained a notch for easy coupling with the electrode cap. The radius of the bottom notch is such that they are capable of easy insertion into an electrode cap while still being able to remain lodged for a long period of time. The final design of the 3D printed holders is shown in Fig. 4.

\subsection{Experimental Studies}

The functionality of the optodes in detecting light transmitted through the tissue was evaluated through experiments. We performed a basic pulse oximetry experiment on the tip of a finger for the electrical test and the working of optode pairs. As the tip of the finger is more transparent than the scalp, a low current level of $3 \mathrm{~mA}$ was supplied to operate the LED in order to avoid detector's saturation and to set the voltage range of the detected signals within the middle of the dynamic range of the fNIRS system. The resulting signal (V) from the photodiode was recorded using our fNIRS system [5] and plotted. From the plot [Fig. 5] 
the amplitude of the detected light at both the wavelengths varies with respect to the changes in blood volume at the tip of the finger due to the heart rate. LED driving current can be increased up to $200 \mathrm{~mA}$. We adjust the current to the LEDs such that the LEDs inject a high-intensity light, within the safe limit to the scalp and the detectors effectively detect back-scattered light from the brain for the fNIRS studies. The resulting signal from the photodiode can be processed using the differential modified Beer-Lambert law to determine the concentrations of oxyhemoglobin and de-oxyhemoglobin.

\subsubsection{User Experience and Signal Quality}

To test the comfort and usability of the final headgear, 8 individual subjects of varying head sizes were asked to wear the headgear for a duration of 30 minutes. 2 subjects were female with long hair. The other 6 subjects were males with short hair. During this time, they were asked to maintain their normal level of activity. Subjects were also asked to perform guided physical activities. The fNIRS signal from the optodes were simultaneously collected with the time tags for the various activities. At the end of the 30 minutes, subjects were asked to complete a short survey that would quantify their experience. In order to ensure that there was no bias, no authors participated. Additionally, the researcher left the room while the participant was taking the survey. Survey responses were given anonymously. The user experience is subjective. However, the average survey results are listed in the Table 1. Per these results, the cap and optodes were rated as being easy to use. Most participants stated that the headgear would be either slightly detrimental to their experience, or not detrimental at all even over a long time interval. The overall subjective experience is that the cap and optodes are relatively comfortable. 
Table 1. Survey Rating Responses

\begin{tabular}{|l|l|l|}
\hline Average Comfort of Optodes & Average Comfort of Cap & Average Usability \\
\hline $8 / 10$ & $8.25 / 10$ & $8.875 / 10$ \\
\hline
\end{tabular}

\subsection{Result}

We have designed fNIRS optodes through iterative human-centered design concept for the portable neuroimaging. Various aspects of the optode design, and $\mathrm{SiPD}$ and LED integration were tweaked in order to ensure maximum applicability to a wide range of users. At the end of this process, effective designs for the source optode and the detector optode were created. The final optodes fit perfectly with the chosen LED and SiPD. Additionally, they vastly simplify the assembly process. The electrical components (once soldered to the correct lead wires) need only to be inserted into the holder and secured with hot glue in order to be functional. These optodes integrating with our laboratory developed control system provide overall SNR between $32 \mathrm{~dB}$ to $70 \mathrm{~dB}$ depending on different experimental conditions. The noise characteristics of the optodes during physical activities were also studied to improve the optode design in each iteration. We found that it was difficult to generalize the SNR level of the optode for the various physical activities as the SNR depends on various factors. However, we found that real-time measurement of the signal level was instrumental for the optode-to-scalp coupling analysis for the various optode design and for the design improvement.

\subsection{Conclusion}

The final design is capable of effectively coupling the source and detector of the fNIRS on the subject's scalp. It was approached by iteratively creating a large number of prototypes. These prototypes were then either modified or abandoned so as to converge to a design that would be effective. This proves that an iterative 
process is an effective approach for implementing the human-centered design. The goal of coupling multiple photodiodes and LEDs to a scalp was achieved through the reliability, durability, and usability of this design. Our optode design framework presented in this paper will be also useful for the further improvement of the optode design in our future studies.

\section{Acknowledgment}

This material is based upon work supported by the National Science Foundation under Grant No. 1539068. The authors would also like to thank Dylan Kennedy and Gozde Cay for their assistance.

\section{List of References}

[1] N. K. Logothetis, "What we can do and what we cannot do with fmri," Nature, vol. 453, no. 7197, pp. 869-878, 2008.

[2] F. Irani, S. M. Platek, S. Bunce, A. C. Ruocco, and D. Chute, "Functional near infrared spectroscopy (fnirs): An emerging neuroimaging technology with important applications for the study of brain disorders," The Clinical Neuropsychologist, vol. 21, no. 1, pp. 9-37, 2007, pMID: 17366276. [Online]. Available: https://doi.org/10.1080/13854040600910018

[3] M. Ferrari and V. Quaresima, "A brief review on the history of human functional near-infrared spectroscopy (fnirs) development and fields of application," NeuroImage, vol. 63, no. 2, pp. 921 - 935, 2012. [Online]. Available: http://www.sciencedirect.com/science/article/pii/ S1053811912003308

[4] M. A. Yücel, J. J. Selb, T. J. Huppert, M. A. Franceschini, and D. A. Boas, "Functional near infrared spectroscopy: Enabling routine functional brain imaging," Current Opinion in Biomedical Engineering, vol. 4, no. Supplement C, pp. 78 - 86, 2017. [Online]. Available: http://www.sciencedirect.com/science/article/pii/S2468451117300697

[5] M. J. Saikia, W. Besio, and K. Mankodiya, "Wearlight: Towards a wearable, configurable functional nir spectroscopy system for noninvasive neuroimaging," IEEE Transactions on Biomedical Circuits and Systems, pp. 1-1, 2018.

[6] M. Firbank, E. Okada, and D. T. Delpy, "A theoretical study of the signal contribution of regions of the adult head to near-infrared spectroscopy 
studies of visual evoked responses," NeuroImage, vol. 8, no. 1, pp. $69-78$, 1998. [Online]. Available: http://www.sciencedirect.com/science/article/pii/ S1053811998903482

[7] M. Cope, "The development of a near infrared spectroscopy system and its application for non invasive monitoring of cerebral blood and tissue oxygenation in the newborn infants," Ph.D. dissertation, University of London, 1991.

[8] J. B. Balardin, G. A. Zimeo Morais, R. A. Furucho, L. Trambaiolli, P. Vanzella, C. Biazoli, and J. R. Sato, "Imaging brain function with functional near-infrared spectroscopy in unconstrained environments," Frontiers in Human Neuroscience, vol. 11, p. 258, 2017.

[9] M. J. Saikia, R. Kanhirodan, and R. Mohan Vasu, "High-speed gpu-based fully three-dimensional diffuse optical tomographic system," International Journal of Biomedical Imaging, vol. 2014, no. 376456, p. 13, 2014. [Online]. Available: http://dx.doi.org/10.1155/2014/376456

[10] S. M. Liao, S. L. Ferradal, B. R. White, N. Gregg, T. E. Inder, and J. P. Culver, "High-density diffuse optical tomography of term infant visual cortex in the nursery," Journal of Biomedical Optics, vol. 17, no. 8, p. 081414, 08 2012. [Online]. Available: http: //www.ncbi.nlm.nih.gov/pmc/articles/PMC3391961/

[11] M. J. Saikia, K. Rajan, and R. M. Vasu, "3-d gpu based real time diffuse optical tomographic system," in 2014 IEEE International Advance Computing Conference (IACC), 2014, pp. 1099-1103.

[12] M. J. Saikia, R. Manjappa, and R. Kanhirodan, "A cost-effective led and photodetector based fast direct $3 \mathrm{~d}$ diffuse optical imaging system," in Diffuse Optical Spectroscopy and Imaging VI. Optical Society of America, 2017, p. 104120Y. [Online]. Available: http: //www.osapublishing.org/abstract.cfm?URI=ECBO-2017-104120Y

[13] H. Dehghani, B. R. White, B. W. Zeff, A. Tizzard, and J. P. Culver, "Depth sensitivity and image reconstruction analysis of dense imaging arrays for mapping brain function with diffuse optical tomography," Appl. Opt., vol. 48, no. 10, pp. D137-D143, Apr 2009.

[14] M. J. Saikia and R. Kanhirodan, "High performance single and multi-gpu acceleration for diffuse optical tomography," in 2014 International Conference on Contemporary Computing and Informatics (IC3I), 2014, pp. 1320-1323.

[15] M. J. Saikia and R. Kanhirodan, "Region-of-interest diffuse optical tomography system," Review of Scientific Instruments, vol. 87, no. 1, p. 013701, 2016. [Online]. Available: https://doi.org/10.1063/1.4939054 
[16] M. J. Saikia and R. Kanhirodan, "Development of dot system for roi scanning," in 12th International Conference on Fiber Optics and Photonics. Optical Society of America, 2014, p. T3A.4. [Online]. Available: http://www.osapublishing.org/abstract.cfm?URI=Photonics-2014-T3A.4

[17] N. M. Gregg, B. R. White, B. W. Zeff, A. J. Berger, and J. P. Culver, "Brain specificity of diffuse optical imaging: Improvements from superficial signal regression and tomography," Frontiers in Neuroenergetics, vol. 2, p. 14, 2010.

[18] M. J. Saikia, R. Manjappa, K. Mankodiya, and R. Kanhirodan, "Depth sensitivity improvement of region-of-interest diffuse optical tomography from superficial signal regression," in Imaging and Applied Optics 2018 (3D, AO, AIO, COSI, DH, IS, LACSEA, LSEC, MATH, pcAOP). Optical Society of America, 2018, p. CM3E.5. [Online]. Available: http://www.osapublishing.org/abstract.cfm?URI=COSI-2018-CM3E.5

[19] D. Agrò, R. Canicattì, M. Pinto, G. Morsellino, A. Tomasino, G. Adamo, L. Curcio, A. Parisi, S. Stivala, N. Galioto, et al., "Design and implementation of a portable fnirs embedded system," in Applications in Electronics Pervading Industry, Environment and Society. Springer, 2016, pp. 43-50.

[20] S. K. Piper, A. Krueger, S. P. Koch, J. Mehnert, C. Habermehl, J. Steinbrink, H. Obrig, and C. H. Schmitz, "A wearable multichannel fnirs system for brain imaging in freely moving subjects," NeuroImage, vol. 85, pp. 64 - 71, 2014, celebrating 20 Years of Functional Near Infrared Spectroscopy (fNIRS). [Online]. Available: http://www.sciencedirect.com/science/article/pii/S1053811913007003

[21] A. Bozkurt, A. Rosen, H. Rosen, and B. Onaral, "A portable near infrared spectroscopy system for bedside monitoring of newborn brain," BioMedical Engineering OnLine, vol. 4, no. 1, p. 29, Apr 2005. [Online]. Available: https://doi.org/10.1186/1475-925X-4-29

[22] E. S. Greenhouse, "Human-centered design," 2012. [Online]. Available: https://aging.ny.gov/livableny/resourcemanual/ demographicandsocialtrends/i9.pdf

[23] C. L. Ventola, "Medical applications for 3d printing: current and projected uses," Pharmacy and Therapeutics, vol. 39, no. 10, p. 704, 2014.

[24] G. T. Klein, Y. Lu, and M. Y. Wang, "3d printing and neurosurgery-ready for prime time?" World neurosurgery, vol. 80, no. 3, pp. 233-235, 2013.

[25] H. Seitz, W. Rieder, S. Irsen, B. Leukers, and C. Tille, "Three-dimensional printing of porous ceramic scaffolds for bone tissue engineering," Journal of Biomedical Materials Research Part B: Applied Biomaterials, vol. 74, no. 2, pp. 782-788, 2005. 


\section{MANUSCRIPT 2}

\section{WearLight: Towards a Wearable, Configurable Functional NIR Spectroscopy System for Noninvasive Neuroimaging}

by

Manob Jyoti Saikia, Walter G. Besio, and Kunal Mankodiya

published in

IEEE transactions on biomedical circuits and systems, vol. 13, no. 1, pp. 91-102,

Feb. 2019. 


\subsection{Abstract}

Functional Near-Infrared Spectroscopy (fNIRS) has emerged as an effective brain monitoring technique to measure the hemodynamic response of the cortical surface. Its wide popularity and adoption in recent time attribute to its portability, ease of use, and flexibility in multi-modal studies involving electroencephalography (EEG). While fNIRS is still emerging on various fronts including hardware, software, algorithm, and applications, it still requires overcoming several scientific challenges associated with brain monitoring in naturalistic environments where the human participants are allowed to move and required to perform various tasks stimulating brain behaviors. In response to these challenges and demands, we have developed a wearable fNIRS system, WearLight which was built upon an Internet-of-Things (IoT) embedded architecture for onboard intelligence, configurability and data transmission. In addition, we have pursued detailed research and comparative analysis on the design of the optodes encapsulating an NIR light source and a detector into 3D-printed material. We performed rigorous experimental studies on human participants to test reliability, signal-to-noise ratio, and configurability. Most importantly, we observed that WearLight has a capacity to measure hemodynamic responses in various setups including arterial occlusion on the forearm and frontal lobe brain activity during breathing exercises in a naturalistic environment. Our promising experimental results provide an evidence of preliminary clinical validation of WearLight. This encourages us to move towards intensive studies involving brain monitoring.

\subsection{Introduction}

Understanding the human brain functionality is among the most far-reaching challenges today. For example, imaging the brain can help to elucidate different clinical manifestation of neurological conditions including Alzheimer's disease, 


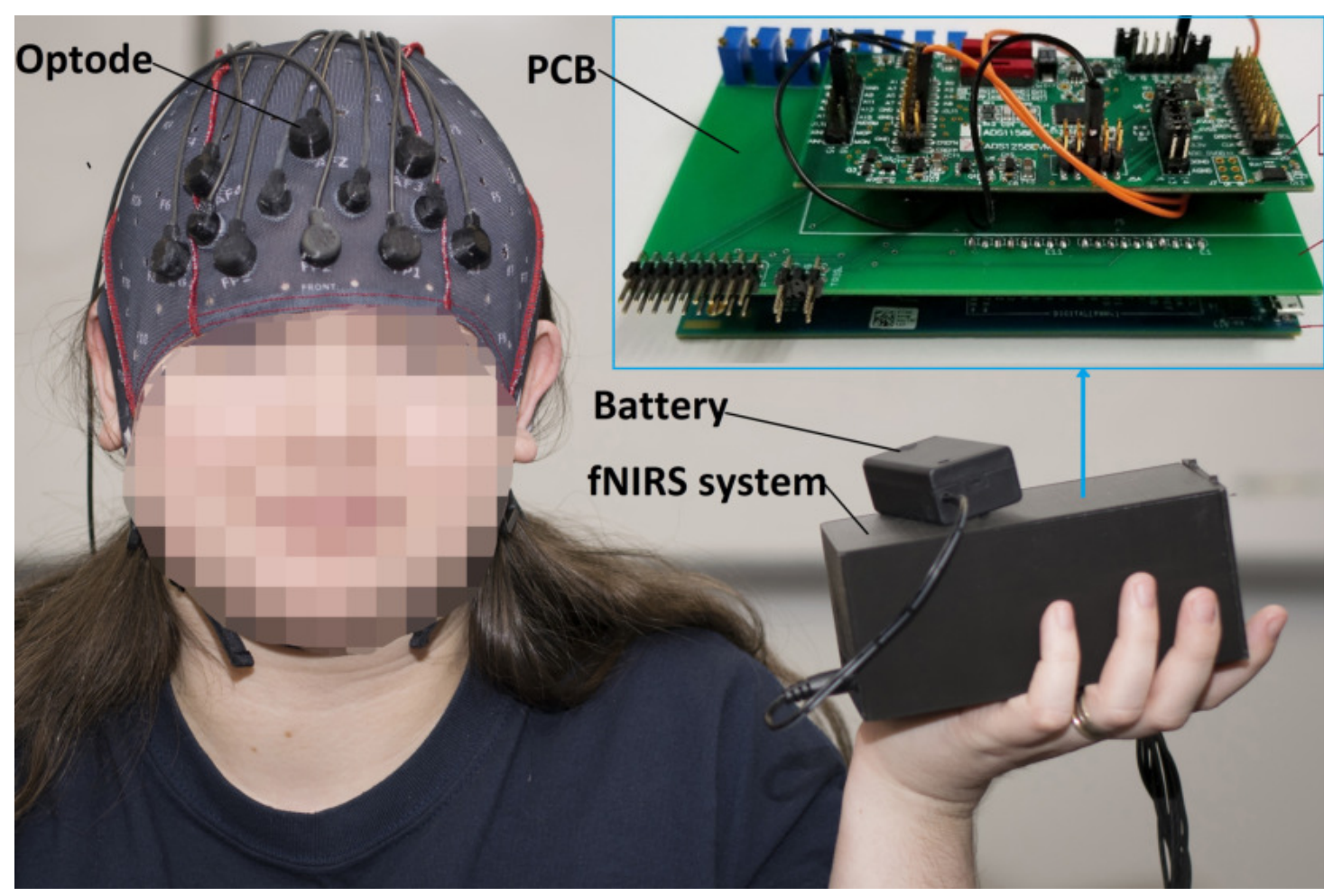

Figure 6. WearLight fNIRS brain imaging system. A participant wearing optodes on the head using an electrode cap to image prefrontal cortex.

Parkinson's disease, Epilepsy, Schizophrenia, mood disorders, anxiety disorders, and brain injuries $[1,2,3,4,5]$. For decades, brain scanning technologies such as functional magnetic resonance imaging (fMRI) have undoubtedly advanced the science of the brain's functional framework. However, fMRI faces limitations and challenges $[6,7,2,8,9,4]$ :

- Low Temporal Resolution: fMRI has high spatial resolution, producing brain images with the voxel size of 1-2 $\mathrm{mm}^{3}$. However, fMRI has limited temporal resolution which is 1 frame per second.

- Non-portability: fMRI is a bulky scanner that requires patients to lay horizontal in a tunnel. fMRI requires a special custom built stationary room.

- Motion Artifact: It is not possible to perform neuroimaging when patients are in their natural positions such as sitting, standing or walking. Ad- 
ditionally, patients are required to stay motionless while undergoing fMRI neuroimaging.

- Non-compatibility: fMRI is not recommended for infants and patients with any ferromagnetic medical implants such as pacemakers, deep brain stimulation (DBS) and some orthopedic implants.

These critical barriers imposed by fMRI motivate the development of other tools which help us to advance the science of the brain's functional architecture. One such tool is functional near-infrared spectroscopy (fNIRS) which is an optical brain imaging technique, based on transmitting NIR light non-invasively from the scalp, and measuring the receiving light passed through the cortex and reflected from the brain $[10,11,12,7,13,14]$. fNIRS is an emerging tool for neuroimaging that shows promise to address a subset of the limitations associated with fMRI. For example, fNIRS has a comparatively higher temporal resolution than fMRI $[15,8,2]$. fNIRS is safe, portable and flexible in regards to monitoring the human brain that is in its natural state without constraining the participants' posture and motion $[9,16,17]$.

In this article, we present the design and development of WearLight, a wearable fNIRS system aimed at advancing the circuit system architecture of fNIRS [Fig. 6]. The system is low-cost, battery operated with continuous wave near infrared spectroscopy (cw-fNIRS) $[13,7,4]$ for real-time monitoring of brains in freely moving humans. The hardware and software were optimized and verified for the functionality, signal quality, and safety. It is also possible to use multiple WearLight systems simultaneously to image brain activities over a large area.

The presented fNIRS system makes the following key contributions:

- Portability \& Wearability: WearLight is a miniaturized fNIRS system made of an embedded controlling unit to provide high-resolution fNIRS data 
at a frame rate of $11 \mathrm{~Hz}$. WearLight's hardware supports up to eight dual wavelength light emitting diodes (LEDs) and sixteen photodiode detectors for the direct illumination and detection respectively. The optodes are placed on the head using a general purpose electrode cap and the portable fNIRS system can be worn on the waist, arm, or other area.

- Wireless \& Configurability: We have designed and developed fNIRS optodes. WearLight provides easy placement of the optodes on the electrode cap, overall low optode-skin contact pressure, electrical isolation, battery operation and optical power within the safe limit. fNIRS channels comprising a source-detector (SD) pair, with adjustable sensitivity. WearLight can be configured wirelessly to set up a montage and also to collect the fNIRS data in real-time.

- Real-time data processing \& visualization: WearLight has the ability to perform data processing and send the data wirelessly to a graphical user interface (GUI) software on a computer. These data processing and displaying services are in real-time.

- Experimental Validation: WearLight is highly stable with respect to the NIR light transmission and detection with an overall low system noise, low channel crosstalk, low drifts and highly sensitive while immune to the background ambient noise. Two sets of experiments, (1) a basic arterial occlusion experiment to measure changes in concentrations of oxy-hemoglobin $\left(\mathrm{HbO}_{2}\right)$ and deoxy-hemoglobin $(H b)$ at the regional muscle, and (2) imaging frontal brain on $(\mathrm{N}=8)$ participants during breathing exercises evaluate the working of the instrument. The typical changes in $\mathrm{HbO}_{2}$ and $\mathrm{Hb}$ during these experiments are presented in the result section of the paper. 

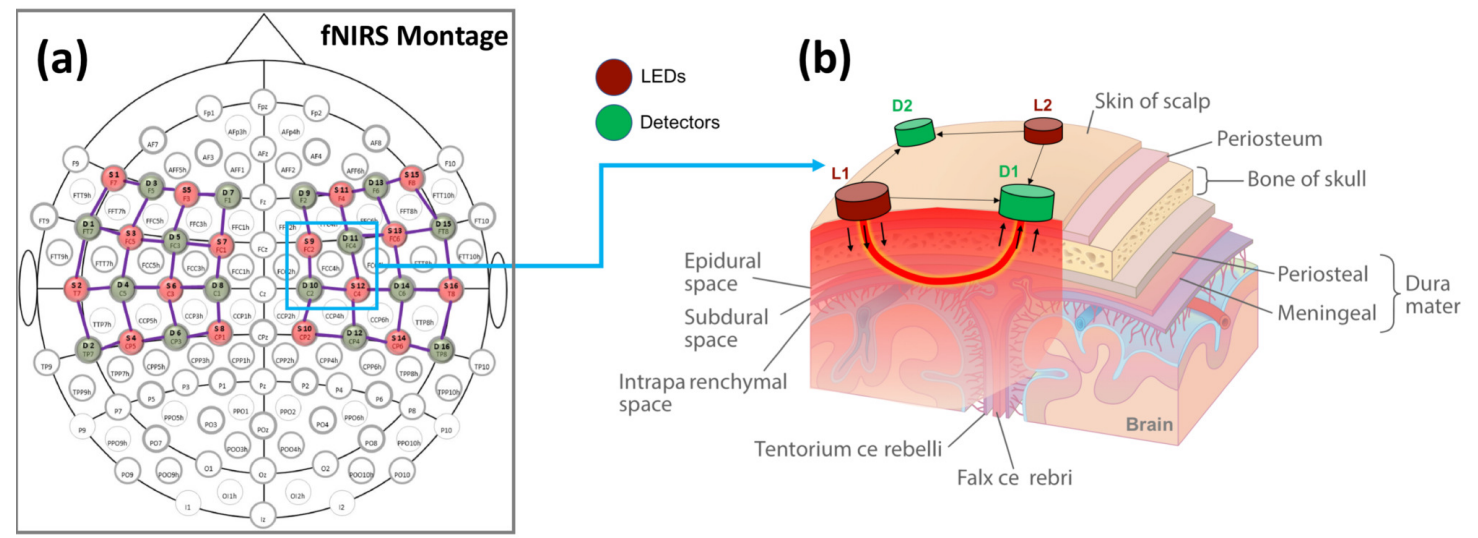

Figure 7. The basic principle of fNIRS. (a) fNIRS montage cap containing NIR light source and detector arrangements, and (b) propagation of NIR light through different tissue layers of a human head from a source to a detector.

The principle and theoretical background of fNIRS are described in Section 2.3.1. The state-of-the-art about fNIRS circuits and systems is described in Section 2.3.2. Section 2.4 describes the design of the WearLight's hardware, involving the detailed explanation of each component. We have performed system-level experiments described in Section 2.5 which involve human participants.

\subsection{Background and State-of-the-Art \\ 2.3.1 fNIRS Principle}

As shown in Figure 7, fNIRS has a close resemblance to EEG, which is another non-invasive brain monitoring method $[18,19,20]$. Both fNIRS and EEG are very similar in terms of their application and appearance because both use a multi-electrode setup covering the scalp to capture the brain activity. The fundamental difference is that fNIRS measures hemodynamic response (pertaining to the dynamics of the cerebral blood oxygenation) while EEG measures the electrical activity of the brain. fNIRS measures changes in chromophore concentration, oxyhemoglobin $\left(\mathrm{HbO}_{2}\right)$ and deoxy-hemoglobin $(\mathrm{Hb})$ in response to the human brain functions non-invasively [10, 21, 22, 23]. Multi-wavelength near-infrared (NIR) light sources are employed to shine light on the scalp of human head that enables 
propagation of the light through the tissue. Each wavelength is absorbed by the chromophores differently [24]. Figure 37 presents the absorption spectra of two important chromophores $\mathrm{HbO}_{2}$ and $\mathrm{Hb}$ for the fNIRS brain imaging. Typically light detectors are used at a distance of 25-40 mm away from the corresponding NIR light source to detect the back-reflected diffused light [25, 26, 27, 28, 29] from the cortical surface of the brain, on the scalp surface.

Figure $7 \mathrm{~b}$ presents the sketch of the cross-sectional distribution of light path in the tissue in a human head. Incident NIR light onto the scalp is scattered and absorbed as it propagates through the scalp, skull, cerebrospinal fluid (CSF), gray matter, and brain, and is detected by a detector on the scalp surface. The detected light intensity variations, at least at two wavelengths, can be used to compute the changes in concentrations of $\mathrm{HbO}_{2}$ and $\mathrm{Hb}$ in response to the activation in the cerebral cortex of the brain. A source-detector (SD) pair can detect brain activity at around the midpoints of the SD pair creating an fNIRS channel. Multiple SD pairs can be used to create large numbers of fNIRS channels to cover the whole brain. Often the fNIRS channels are time, frequency and/or spatially multiplexed in order to simultaneously measure possible fNIRS channels to increase the frame rate of the imaging $[14,17,30]$. Using high density (HD) fNIRS measurement, it is also possible to reconstruct 3D distributions of the chromophore concentration of the brain $[31,25,32,33]$. Among the three fNIRS implementations, time-resolved, frequency domain and continuous wave (cw) technique, cw-fNIRS based on using constant amplitude NIR light intensity is the most practical for portable brain imaging for its simplicity $[12,34,35,17,36,37]$.

The Modified Beer-Lambert Law (MBLL) is the most commonly employed mathematical model to estimate the relative changes in hemoglobin $\left(\triangle \mathrm{HbO}_{2}\right.$ and $\Delta H b)$ concentration $[38,23,11,39]$. 


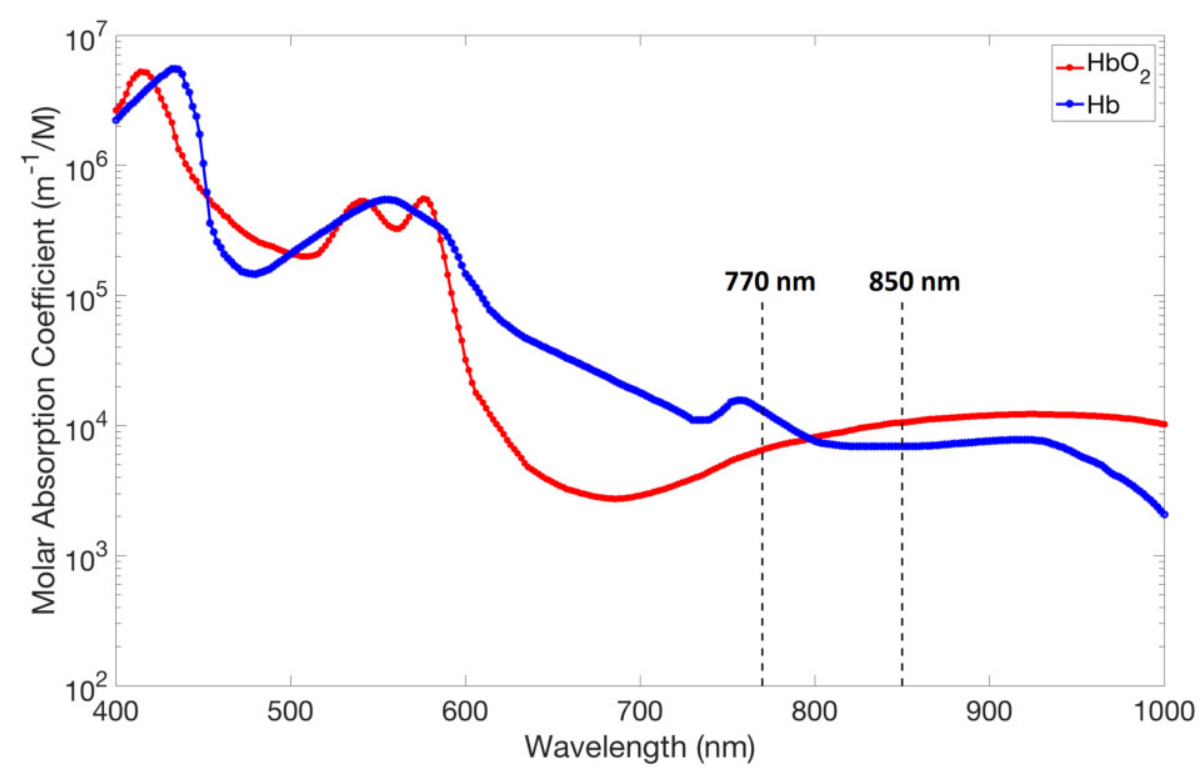

Figure 8. The plot of absorption spectra of oxy-hemoglobin $\left(\mathrm{HbO}_{2}\right)$ and deoxyhemoglobin $(\mathrm{Hb})$ of tissue, represented in terms of molar absorption coefficients. Experimental data were taken from OMLC [24] . The wavelengths of the LEDs used for our fNIRS system are indicated by the vertical dashed line.

The oxy-hemoglobin and deoxy-hemoglobin concentration changes, $\Delta \mathrm{HbO}_{2}$ and $\Delta H b$ can be calculated as $[40,41,23]$,

$$
\begin{gathered}
\Delta H b O_{2}=\frac{\alpha_{H b}^{\lambda_{1}} \frac{\Delta A^{\lambda_{2}}}{L^{\lambda_{2}}}-\alpha_{H b}^{\lambda_{2}} \frac{\Delta A^{\lambda_{1}}}{L^{\lambda_{1}}}}{\alpha_{H b}^{\lambda_{1}} \alpha_{H b O_{2}}^{\lambda_{2}}-\alpha_{H b}^{\lambda_{2}} \alpha_{H b O_{2}}^{\lambda_{1}}} \\
\Delta H b=\frac{\alpha_{H b O_{2}}^{\lambda_{1}} \frac{\Delta A^{\lambda_{2}}}{L^{\lambda_{2}}}-\alpha_{H b O_{2}}^{\lambda_{2}} \frac{\Delta A^{\lambda_{1}}}{L^{\lambda_{1}}}}{\alpha_{H b O_{2}}^{\lambda_{1}} \alpha_{H b}^{\lambda_{2}}-\alpha_{H b O_{2}}^{\lambda_{2}} \alpha_{H b}^{\lambda_{1}}}
\end{gathered}
$$

where $\alpha, A$ and $L$ are molar absorption coefficients, attenuation of light and mean path length of detected photon respectively measured at two wavelengths $\lambda_{1}$ and $\lambda_{2}$.

\subsubsection{State-of-the-Art: fNIRS System Design}

Typically, an fNIRS system consists of a table-top instrument connected to a desktop computer and optical fibers with a montage cap. The instrument is controlled by a computer software and the montage cap interfaces human brain to 
the fNIRS system. These fNIRS systems are mostly operated in the laboratory environment for neuroscience research. Although optical-fiber-based fNIRS systems have some advantages for emitting and collecting light, they limit the human's movements and the fibers exacerbate motion artifacts contamination. While the spatially restrained settings are feasible for bedside monitoring, fibers degrade the quality of brain imaging while participants perform natural physical activities or imaging an infant $[16,30,12]$.

To overcome these constraints, a new generation of wireless fNIRS systems that can be worn and carried by the participant is desirable. To achieve a wearable fNIRS system, it needs to be a low-power battery-operated wireless system. The system is required to be small and lightweight, while providing a greater number of optodes and their corresponding fNIRS channels, without sacrificing the frame rate of the system ( $>6 \mathrm{~Hz}$ preferable). Also, the sensitivity and dynamic range of the system needs to be high enough to support a wide range of SD distances $(2 \mathrm{~cm} \leq \mathrm{SD}$ distance $\leq 4 \mathrm{~cm})$ and to detect very low intensity optical signals when the SD distance on the hairy head is greater. If the wearable fNIRS system is compatible with EEG, it can provide the added benefit of multi-modal imaging. Additionally, real-time configurability, control, data acquisition, data processing and displaying results are beneficial.

In recent years, research and publications on fNIRS brain imaging are growing [7]. However, most of are based on clinical aspect of the fNIRS system [3, 2, 4, 5, 16, 19, 8, 20], data analysis [39, 42, 43] and its usage in BCI applications or neuroimaging $[44,45,13,34,9,46]$. Some studies on the hardware aspect of the fNIRS system have been published recently $[47,48,49,50,37,35,51,52]$ and increasing interest in multi-channel portable fNIRS systems are observed. There are few studies that have attempted development for portable wearable fNIRS 


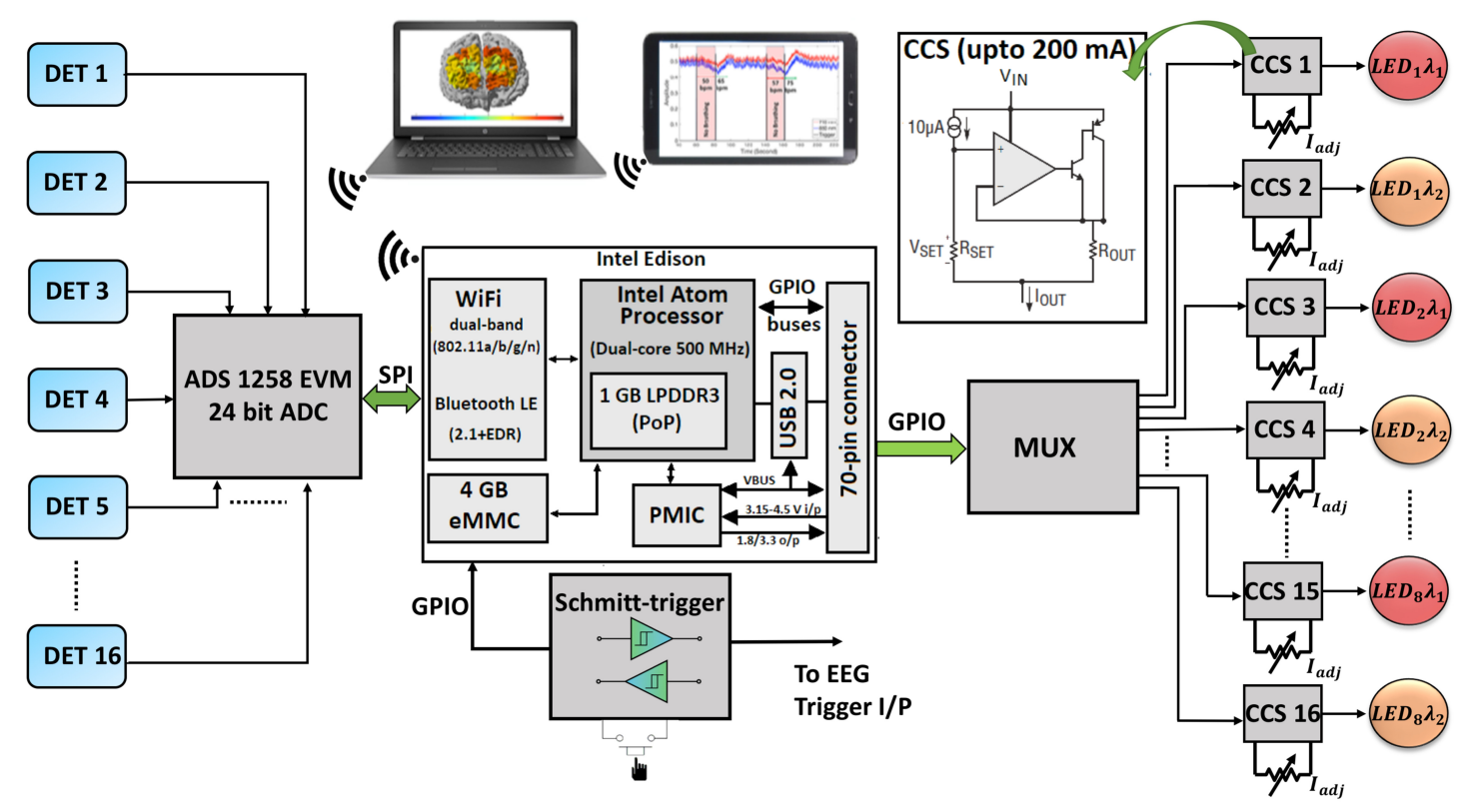

Figure 9. A simplified block diagram showing the hardware components of the WearLight fNIRS system. A single WearLight module supports eight dual wavelength LEDs and sixteen detectors.

brain imaging to free up the participant's head position $[30,36,53,17,35,50,54]$. These systems certainly have some advantages. However, each of them suffers several drawbacks: (1) fewer fNIRS measurement channels, (2) low frame rate, (3) low dynamic range (ADC bit resolution), (4) incompatible for EEG electrode placement, (5) less sensitive ( $\leq 3 \mathrm{~cm}$ SD separation), (6) suitable only for limited regions of the head, or (7) heavy to carry around with the wire connected to PC, as listed in the Table 3 in the section 2.6.

\subsection{WearLight fNIRS System Design}

An fNIRS system that can monitor brain functional activities continuously in an unconstrained manner, requires the system to have a small form factor, easily wearable, light, configurable, and battery-operated and preferably wireless. As the number of fNIRS channels increases in the system, meeting these hardware requirements for such a system for the long-term brain monitoring becomes challenging. 
In addition, for a multi-channel portable wireless fNIRS system, the real-time configuration, and data transmission, processing and visualization are demanding. However, the multi-channel fNIRS system has the advantage of topographic imaging of an overall large portion of the brain that provides the opportunity for comprehensive neuroscience studies.

In this research, we have focused on the development of a multi-channel fNIRS system, WearLight with the capabilities of wireless connectivity and configurability while the participant wears the system. It is intended to be a portable system that can be used during physical activities or at rest. In order to achieve this goal, we have developed a modern fNIRS hardware incorporating the Intel Edison embedded computing platform that enables us to configure, operate and acquire data wirelessly to monitor hemodynamic states of the brain in real-time.

Our software architecture connects to our fNIRS hardware system using a computer or Android tablet through WiFi and interacts to send configuration settings for the montage and also to receive fNIRS data. The architecture of the WearLight system comprises three distinctive parts: fNIRS control unit, a set of optodes along with a montage cap, and a data processing unit (computer/tablet).

Figure 9 presents the main functional units of our fNIRS system. Figure 6 shows a participant holding the control unit and optodes on the head. The optode montage cap holds the light sources aFnd detectors in an appropriate geometry on the region of the brain intended to be monitored. The optodes are interfaced to the control unit via a bundle of flexible biocompatible electric cables using a dedicated connector. The fNIRS control unit can handle up to 8 dual-wavelength LEDs and 16 detectors creating maximum of $8 \times 16=128$ fNIRS channels. However, the usable channels are decided by the optode's placement on the montage cap that consists of a combination of light sources and detectors, and the SNR of the fNIRS 
channels.

A host computer, as a data processing unit, connects to the fNIRS control unit via authentication and performs bi-directional communication to synchronize the operations such as switching the light sources, optical measurements at two wavelengths, data transmission, fNIRS data processing and hemodynamics response visualization. In addition, the control unit saves optical raw measurement data in a file in its own memory for the future use.

\subsection{1 fNIRS Controlling Unit}

The portable fNIRS controlling unit is the core of WearLight. To minimize noise and electrical crosstalk between analog signals, and analog and digital circuits, and electrical safety, the hardware is a multi-layer and multi-power supply architecture. This modular approach helps in testing and debugging, as well as for the future hardware upgrades. We have fabricated three such fNIRS controlling units. They can be operated independently or configured to work together in a synchronized manner to increase the fNIRS channel count.

Each fNIRS controlling unit consists of three printed circuit boards (PCBs) sandwiched together: (1) embedded system PCB, (2) analog circuit PCB and (3) digitization PCB. It also has a dedicated power supply for each PCB. The fNIRS controlling unit is enclosed in a $3 \mathrm{D}$ printed box $\left(13.5 \times 6 \times 8.4 \mathrm{~cm}^{3}\right)$ having access to all of the connector ports [Fig. 6]. The user can wear this unit using a waist or arm band. Optodes from the montage cap and battery are connected to the control unit through two separate connectors. Once powered ON the fNIRS system boots up and goes to idle mode. A computer or a tablet can be used to connect to the control unit wirelessly in order to send the command to start the fNIRS data collection and to visualize data in real-time wirelessly. 


\section{Embedded System PCB}

The bottom-most PCB of the fNIRS controller consists of an Intel Edison System on Chip $(\mathrm{SoC})\left(3.55 \times 2.5 \times 0.39 \mathrm{~cm}^{3}\right)$ along with the necessary peripheral devices and interfaces. We have chosen the Edison for its small form factor, lowpower consumption, multiple connectivity options, and availability of a broad set of I/O support, while a powerful computational capability for the wearable device.

The embedded system is the heart of the controller that is responsible for the synchronization of the tasks of the fNIRS system. It connects to a host computer wirelessly and communicates back and forth throughout the fNIRS data acquisition. The embedded system performs the high speed bi-directional digital data communication with the analog to digital converter (ADC) present on the digitization PCB. The embedded system sends the ADC configuration settings and reads 32 bit (24 bit $\mathrm{ADC}$ and 8-bit status) data at every measurement event. It also performs basic digital data conversion, processing, formatting, wireless data transmission to the host computer and stores data in a file for future use. Thus WearLight works as an IoT device having the capability of real-time configurability, control, data acquisition, data processing and displaying results. The Intel Edison, as a single board computer in the WearLight system also computes $\Delta \mathrm{HbO}_{2}$ and $\triangle H b$ in real-time. However, we perform intensive data processing in our MatLab based GUI software in the host computer for better performance. The host computer acquires data wirelessly and performs basic data processing simultaneously to display $\Delta \mathrm{HbO}_{2}$ and $\Delta \mathrm{Hb}$ in real-time. Advanced data analysis processing, such as removal of the noise, correction of motion artifact and baseline shifting etc., and topographic plots are performed offline in the host computer. 


\section{Analog Circuit PCB}

The middle PCB has the circuitry for the constant current source (CCS), multiplexer, current adjustment $\left(I_{a d j}\right)$, high-speed sync out and trigger. This PCB also routes the bottom digitization PCB to the top embedded system PCB. The analog circuit PCB contains connectors for the optodes, trigger input switch, and trigger out.

The analog circuit PCB contains the circuitry for the LED current driver to adjust the current accurately for each LED at each wavelength. Figure 9 also shows the circuit diagram of the CCS that provides current regulation within 10 $\mathrm{ppm} / \mathrm{V}$. The potentiometer $\left(R_{S E T}\right)$ on the analog $\mathrm{PCB}$ as shown in figure 3 , serves to adjust the constant current $\left(I_{a d j}\right)$ for the LEDs to emit a stable optical power $(<5 \mathrm{~mW})$ within the safe range [55]. The current can be set between $0.5 \mathrm{~mA}$ and $200 \mathrm{~mA}$. We have used a low ON resistance and low OFF leakages 16-channel analog multiplexer (CD74HC4076) to bias and switch the CCS of the LEDs. The regulated current from the CCS is given by $I_{O U T}=10 \mu A \times R_{S E T} / R_{O U T}$, which is independent of the variation in the supplied bias voltage. Hence, resistors with the same temperature coefficient, in the resistor network allow us to apply a regulated current independent to the other circuit variabilities. The analog circuit PCB can handle up to 16 CCSs.

\section{Digitization PCB}

The topmost PCB consists of an ADC along with its necessary circuit components to test and configure the rate of digitization and data transfer with the embedded system. We have used a 24-bit Delta-sigma $(\Delta \Sigma)$ ADC from Texas Instrument (ADS1258). The ADC utilizes the fourth-order modulator followed by a fifth-order sinc digital filter optimized for the low-noise performance. We configured the ADC for 16 single-ended inputs so that a single fNIRS module supports up 


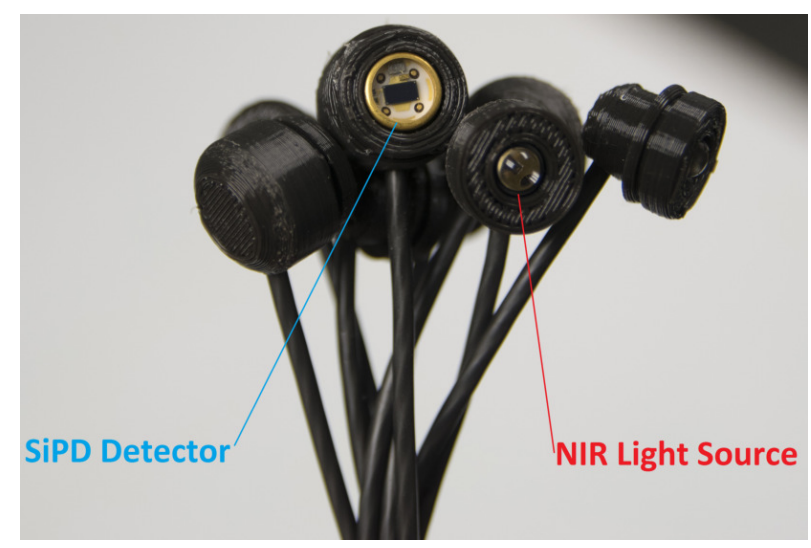

Figure 10. 3D printed optode design. Optodes made of multi-wavelength LEDs (MTMD7885NN24) and Si-PD detectors (ODA-6WB-500M detectors).

to 16 detectors. This top data acquisition digitization PCB communicates with the bottom embedded system via serial digital communication, SPI (Serial Peripheral Interface).

\subsubsection{Optodes (NIR Light Source and Detector)}

The optodes for fNIRS are of two types: 1) the NIR light sources at multiple wavelengths and 2) light detectors to receive the back-reflected light from the brain. The optodes are the interface between the scalp surface and the fNIRS hardware. The optodes are the most critical parts of the fNIRS system since the overall signal quality of the fNIRS imaging greatly relies on the type of optodes used, proper optodes-scalp coupling, and the electrical stability. The human scalp has an irregular geometry having a curved surface. The coupling of the optodes to the scalp through the hair is challenging, as the source optodes must be placed orthogonally to the scalp surface in order to maximize the injection of the NIR light through the tissue and to prevent leakage of direct NIR light to the neighboring detectors. Similarly, the detectors should also be orthogonal to the scalp surface only to collect the back scattered NIR light from the deep tissue, isolating the possible direct ambient and NIR light from the neighboring source. At the 
same time, the participant should feel comfortable wearing the optodes for brain monitoring experiments that tend to run for a significant period of time.

Often detectors get saturated or the signal to noise ratio (SNR) level plummets due to poor coupling of the optodes to the scalp or motion artifacts $[42,39]$. In that case, the severely affected fNIRS channels need to be discarded from further data processing. In WearLight, we have adopted an optical fiberless optodes design that requires less electric power in order to work with the battery operated fNIRS control unit for long-term brain monitoring. Therefore, we use dual wavelength LEDs as an NIR source and photodiode as a detector for our optode design. The LEDs and detectors are housed in a 3D printed holder so that optodes can be appropriately placed on a montage cap and detached easily. This also helps for the multi-modal EEG-fNIRS imaging, where both the EEG electrodes and fNIRS optodes are required to be placed at the same time using a common montage cap $[18,43,51]$. Detectors are positioned $30-40 \mathrm{~mm}$ away from the source in order to receive fNIRS signals from the cortical surface of the brain.

Motion artifact in fNIRS brain imaging is unavoidable. Head movement causes a decoupling of the optode and scalp, resulting in high-frequency spike and baseline shifting of the measured signal. This problem is sometimes solved by simply rejecting all the trials where the motion artifacts are dominant. However, in case of an infant, clinical patient and children, where the number of trials is limited, and fNIRS brain imaging of a freely moving subject, rejection of trials might not be feasible. Hence, intensive post-processing is performed in order to remove motion artifact from the measurements using the inherent characteristics of the amplitude and frequency of the signal due to the artifact. Many post-processing approaches are proposed to correct for motion artifacts and each of them has their own benefits [56]. Some of them are principal component analysis (PCA) [57], independent 
component analysis (ICA) [58], Kalman filtering [59], correlation-based signal improvement (CBSI) [60], wavelet filtering [61] and spline interpolation [62].

\section{Light sources - LEDs}

We use LEDs as the NIR light source over lasers. The broader emission spectra $(\Delta \lambda=35 \mathrm{~nm})$ as opposed to the sharp peaked laser diode $(\Delta \lambda=1 \mathrm{~nm})$ and the incoherent and uncollimated optical output of these LEDs make the use of LED safe, allowing direct contact with the skin. Also, the power consumption of LEDs is much less than the laser source. On the other hand, laser light sources often require temperature monitoring and cooling systems to remove extreme heating of the semiconductor junction and optical fibers to carry light to tissue. Furthermore, the operation of lasers demands additional care as it can cause potential injuries to eyes. Hence, LEDs are appropriate for our portable battery operated fNIRS system that reduces the overall system cost and hardware requirements while making the optode design compact. Also, LEDs as an NIR source are suitable for brain monitoring of vulnerable population of newborns.

We have designed source optodes using three wavelengths (750, 810 and 850 nm) LED package MTMD7885NN24 from Marktech Optoelectronics. We have selected two wavelengths 750 and $850 \mathrm{~nm}$ out of the three available wavelengths. The spectral half width $(\Delta \lambda)$ of both 770 and 850 wavelengths are $35 \mathrm{~nm}$. These two wavelengths are sufficient to resolve concentrations of two chromophores, $\mathrm{HbO}_{2}$ and $H b$. Also, crosstalk between these two wavelengths is less significant with respect to other wavelengths combinations as seen in Figure 37. Although $\Delta \lambda$ is wider compared to the diode lasers, $35 \mathrm{~nm}$ is narrow enough to resolve two chromophores, since absorption spectra are relatively flat around these wavelengths [vertical dashed line shown in Figure 37]. Also, the isosbestic point, $810 \mathrm{~nm}$ lies between $770 \mathrm{~nm}$ and $850 \mathrm{~nm}$, at which $\mathrm{HbO}_{2}$ and $\mathrm{Hb}$ have identical absorption 
coefficients as evident from Figure 37. The LED driving current is set by CCS as described in section 2.4.1 such that the maximum optical power is below $5 \mathrm{~mW}$ when the LED is ON.

\section{Photodetectors}

We use silicon photodiode (Si-PD) detectors, ODA-6WB-500M (Opto Diode Cop.) to detect the optical signals that are reflected from the cortex and other tissues. Si-PD detectors do not need the high voltage supplies that an avalanche photodiode (APD) or photomultiplier tube (PMT) detectors do. Also, Si-PDs are smaller and cost-effective for covering a large portion of the head. They are also suitable for high-speed fNIRS data collection for higher imaging frame rates. The ODA-6WB-500M has high responsivity of about $250 \mathrm{~V} / \mu \mathrm{W}$. It has a cylindrical package which is convenient for fabricating holders for the cap.

We have designed 3D printed LED and detector holders such that the optodes can be easily attached and detached from the montage cap. The circuitry and connections are housed in the internally shielded optode holders. This feature allows us to configure the optode locations on the head depending on the imaging area and experimental protocol and to place the optodes alongside the EEG electrodes. Figure 10 shows the final version of the optode based on dual-wavelength LEDs and ODA-6WB-500M used for our experimentation.

In fNIRS brain imaging, recording high SNR fNIRS signals from the subject's head covered with dense, thick and dark hair is challenging. If the optodes are designed on a sensor pad $[37,30,36,53,35,50,54]$, placement of optodes on the hairy head is even more challenging. This design also limits placement of EEG electrodes alongside the fNIRS optodes. Our optodes are discrete elements and the overall sensitivity of the WearLight's fNIRS channels are high, that allow us to place optodes on the hairy head beside EEG electrodes to collect EEG and fNIRS 
signals simultaneously. We use water-soluble gel to keep hair pushed out of the way to enhance the optode-scalp coupling. Thus our lightweight fiberless optode (3.6 gm per optode with the connecting cable) improves coupling the sources and the detectors to the head and provides a better comfort level for long-term brain monitoring. Also, this design suffers from fewer motion artifacts compared to optical fiber based optode.

The effective quantization signal to noise ratio (SNR) of the WearLight's hardware is $131.8 \mathrm{~dB}$ and the noise level is $2.8 \mu V_{R M S}$. However, the SNR of each fNIRS channels during an experiment depends on various factors such as LED light intensity and wavelength, SD distance, noise equivalent power (NEP) of the detector, physiological noise, ambient noise, sampling rate, skin tone, thickness and color of hair and optode-scalp coupling. We have experimentally evaluated the achievable true SNR of the WearLight's fNIRS channels. In a typical experiment, SNR of the individual fNIRS channels varies between $32 \mathrm{~dB}$ and $70 \mathrm{~dB}$ depending on the mentioned factors. WearLight helps to improve SNR of the fNIRS channels by using the GUI software that displays computed SNR of the individual fNIRS channels during the initial calibration process prior to the actual experiment. It allows us to visualize the coupling status and overall noise level of all the channels. We adjust for the optimum scalp coupling, and to maximize the SNR of all fNIRS channels in the calibration process in order to achieve a maximum number of usable fNIRS channels. We also examine the presence of the photoplethysmographic signal (due to the cardiac cycle) superimposed on the fNIRS signal to verify the effective coupling of the optode to the scalp. We suggest WearLight user to use another layer of opaque head cap on the optode cap to minimize the influence of background radiation if it is necessary. 


\subsection{Results}

We have performed both basic validation and clinically-relevant experiments on eight healthy adult participants to evaluate WearLight system. The first experiment was to monitor the changes in hemoglobin concentration of regional muscle. We have compared our results with the results published by other researchers to verify the working of WearLight. The second experiment involved imaging prefrontal cortex of participants performing breathing exercises. This experiment allowed us to image the hemodynamic response of the brain in response to the breathing exercise. Also, the observed heart rate variation patterns, superimposed on the fNIRS signal, supported the expected heart rate variation in the breathing exercises.

We have used MBLL $[38,23,40,41,11]$ and Equ. 1 to compute $\Delta \mathrm{HbO}_{2}$ and $H b$ over time. The raw fNIRS measurement data from the brain carries other physiological noises such as heart beat $(1 \sim 1.5 \mathrm{~Hz})$, respiration $(0.2 \sim 0.5 \mathrm{~Hz})$ and Mayer waves $(\sim 0.1 \mathrm{~Hz})$ etc. Hence, we have used second-order Butterworth bandpass filter, $\left(f_{\text {cutoff,low }}=0.01 \mathrm{~Hz}\right.$ and $\left.f_{\text {cutoff,hi }}=0.25 \mathrm{~Hz}\right)$ to remove these noises present in the fNIRS signal.

\subsubsection{Experiment 1: Arterial Occlusion Experiment}

The first experiment with the WearLight fNIRS system was to measure the changes in hemoglobin concentration at the regional muscle (forearm) due to the arterial occlusion. This experiment evaluates the working of the hardware and software of our fNIRS system. An optode pair was placed on the forearm as shown in Figure 11 (a). Since the forearm tissue is more transparent than the scalp tissue, this experiment requires less optical power from the LED in order to set the detector in the middle of the dynamic range. A sphygmomanometer cuff was rapidly inflated to $180 \mathrm{mmHg}$ around the upper arm, just above the elbow to exclude blood 

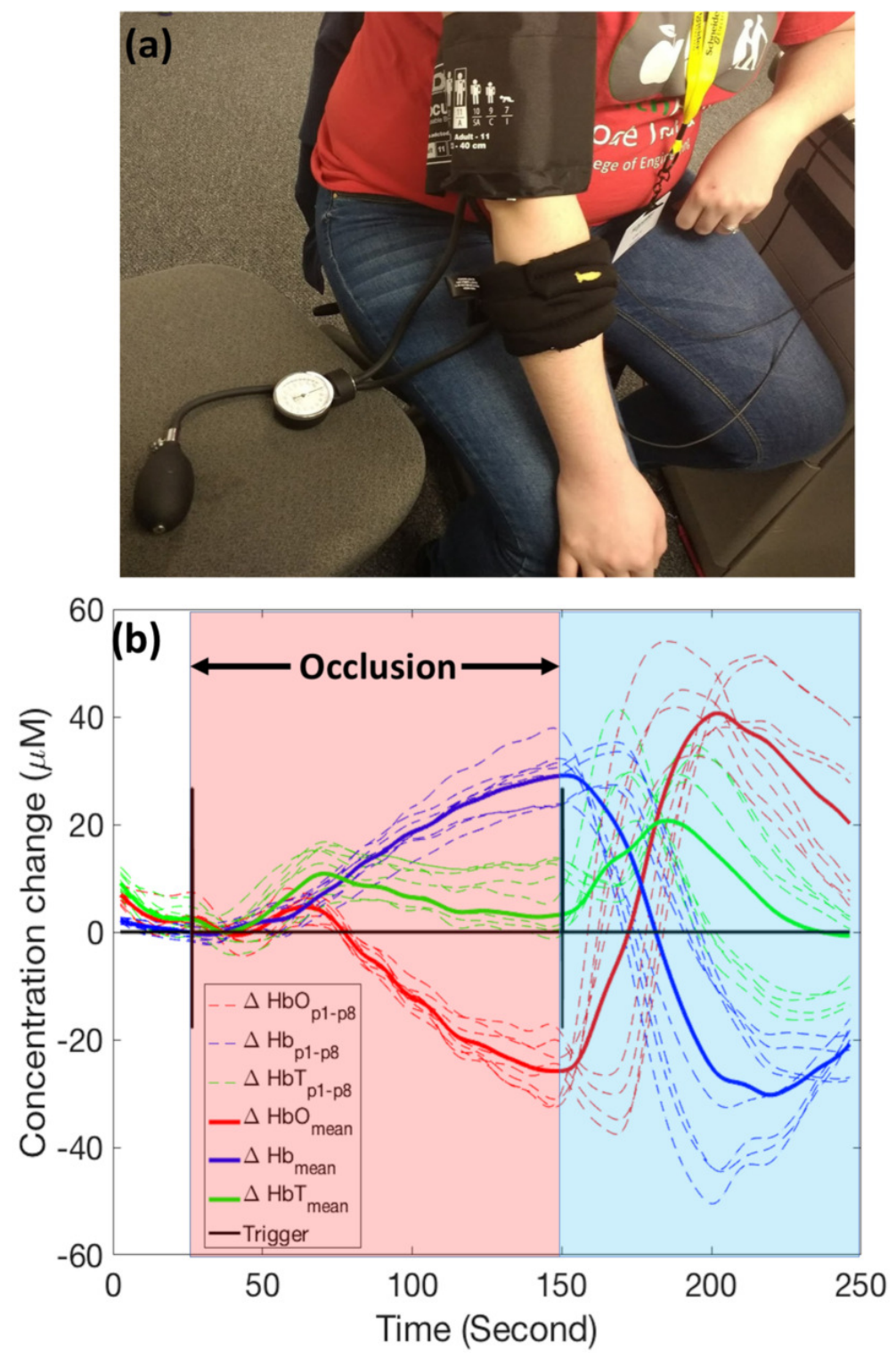

Figure 11. (a) Experimental set-up for arterial occlusion experiment, a participant is wearing a pair of optodes and sphygmomanometer cuff, and (b) forearm muscle response during arterial occlusion experiment. $\Delta H b O_{p 1-p 8}, \Delta H b_{p 1-p 8}$ and $\Delta H b T_{p 1-p 8}$ are the results from the participant no. 1 to participant no. 8 respectively and $\Delta H b O_{\text {mean }}, \Delta H b_{\text {mean }}$ and $\Delta H b T_{\text {mean }}$ are the mean response in the experiment.

flow to the hand for not more than 120 seconds for the participant's comfortability. As evident from the time interval between the occurrences of two trigger events corresponds to the starting and ending of occlusion respectively as shown 
in Figure 11 (b). Figure 11 (b) also presents the changes in hemoglobin concentration, oxy-hemoglobin $\left(\mathrm{HbO}_{2}\right)$ and deoxy-hemoglobin $(\mathrm{Hb})$ of eight participants (dashed lines) over time, derived from the raw experimental data collected from eight participants. The mean response of the $\left(\mathrm{HbO}_{2}\right)$ and $(\mathrm{Hb})$ changes are presented with bold solid lines. This result depicts a decrease in $\mathrm{HbO}_{2}$ concentration and a simultaneous increase in $H b$ concentration due to depletion of locally available hemoglobin in the forearm after trigger event 1. It is also apparent that the release of the occluding cuff, after trigger event 2, blood volume increases rapidly, resulting in an increase in $\mathrm{HbO}_{2}$ concentration and a quick washout of $\mathrm{Hb}$. We have also observed that our results show hemoglobin concentration change similar to the result published by Shang et al.[63], where the measurements were taken using a portable diffuse correlation spectroscopy (DCS) system on the calf muscle during occlusion. The maximum total hemoglobin concentration change (Max. $\triangle H b T$ ) in this experiment (Exp. 1) for the eight subjects are also presented in Table 4 .

\subsubsection{Experiment 2: Prefrontal Cortex fNIRS Experiment}

The experimental setup for imaging prefrontal cortex of the brain using our fNIRS system on 8 participants was similar to the placement of optodes on the head as shown in Figure 6. The participants wore four pairs of fNIRS optodes to cover the prefrontal cortex area of the brain. Participants were trained and asked to perform breathing exercises. The experimental protocol involved performing relaxed deeper breathing for about 60 seconds and after an occurrence of the first trigger, participants were asked to perform breath retention after the exhalation was complete. The no breath period lasted for about 20 seconds depending on the participants' comfortability level. After the end of no breath period, a second trigger event occurred and the participants started breathing again. The time 


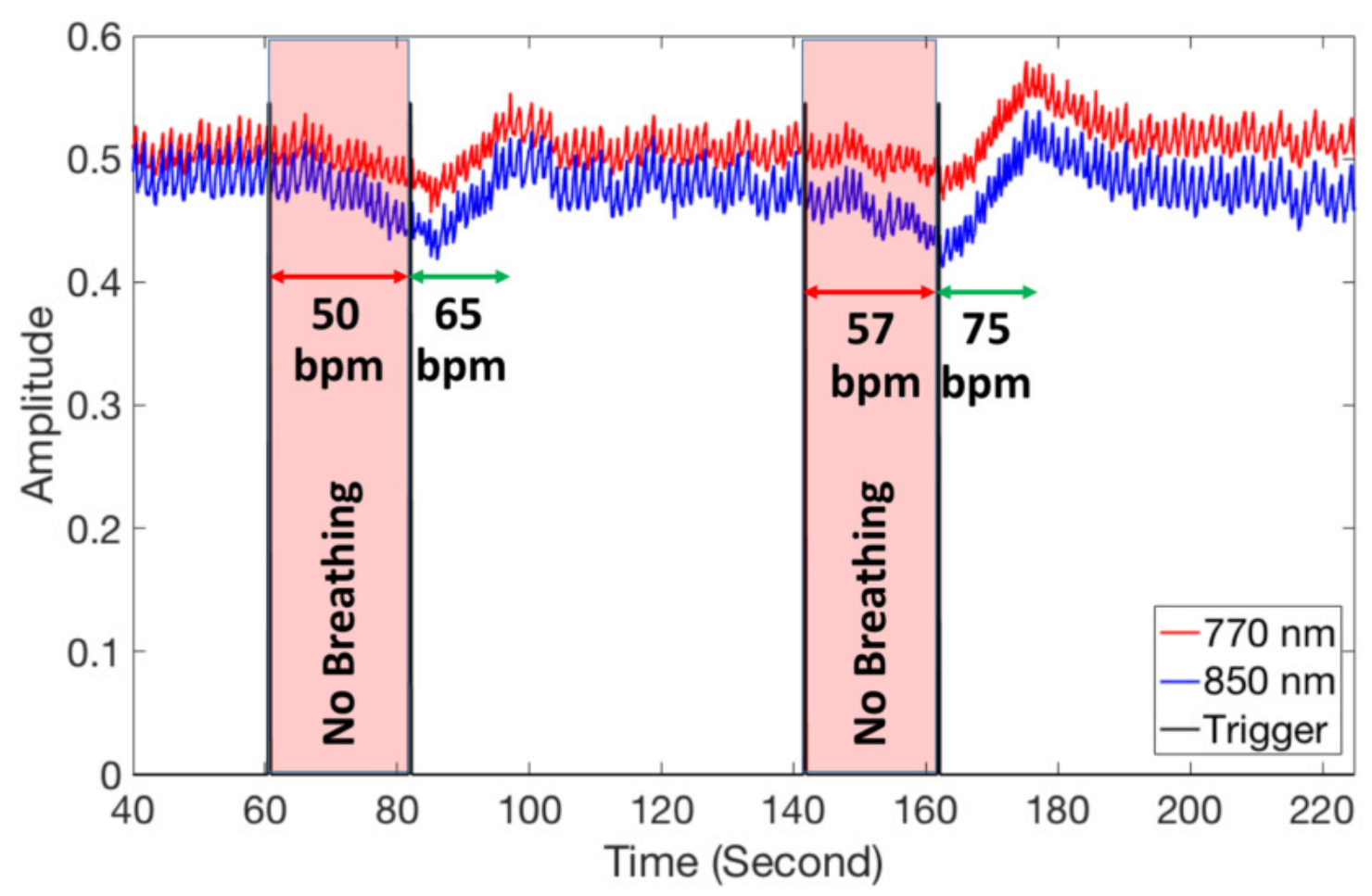

Figure 12. An experimental raw fNIRS data channel during breathing experiment, collected from a participant.

interval between the two vertical trigger lines in Figures 12 and 13, represents the no breath periods. This was continued for several cycles. Figure 12 shows the plot of raw experimental data from an fNIRS channel, collected from a participant that contains signals from both the wavelengths 770 and $850 \mathrm{~nm}$.

We have observed that the raw experimental data from the prefrontal cortex also carried the pulsation caused by heart beats along with the fNIRS signal. Hence, we have also incorporated an estimation of heart rate variability (HRV) from these pulse waves in our studies. As shown in Figure 12, at the first no breath period of 17.9 seconds, 15 peaks were detected corresponding to a slow heart rate of 50 beats per minute (bpm), whereas at the beginning of next start breathing period of 13.8 seconds, 15 peaks were observed that corresponds to an increased heart rate of $65 \mathrm{bpm}$. Similarly, $57 \mathrm{bpm}$ and $75 \mathrm{bmp}$ heart rates were observed for 


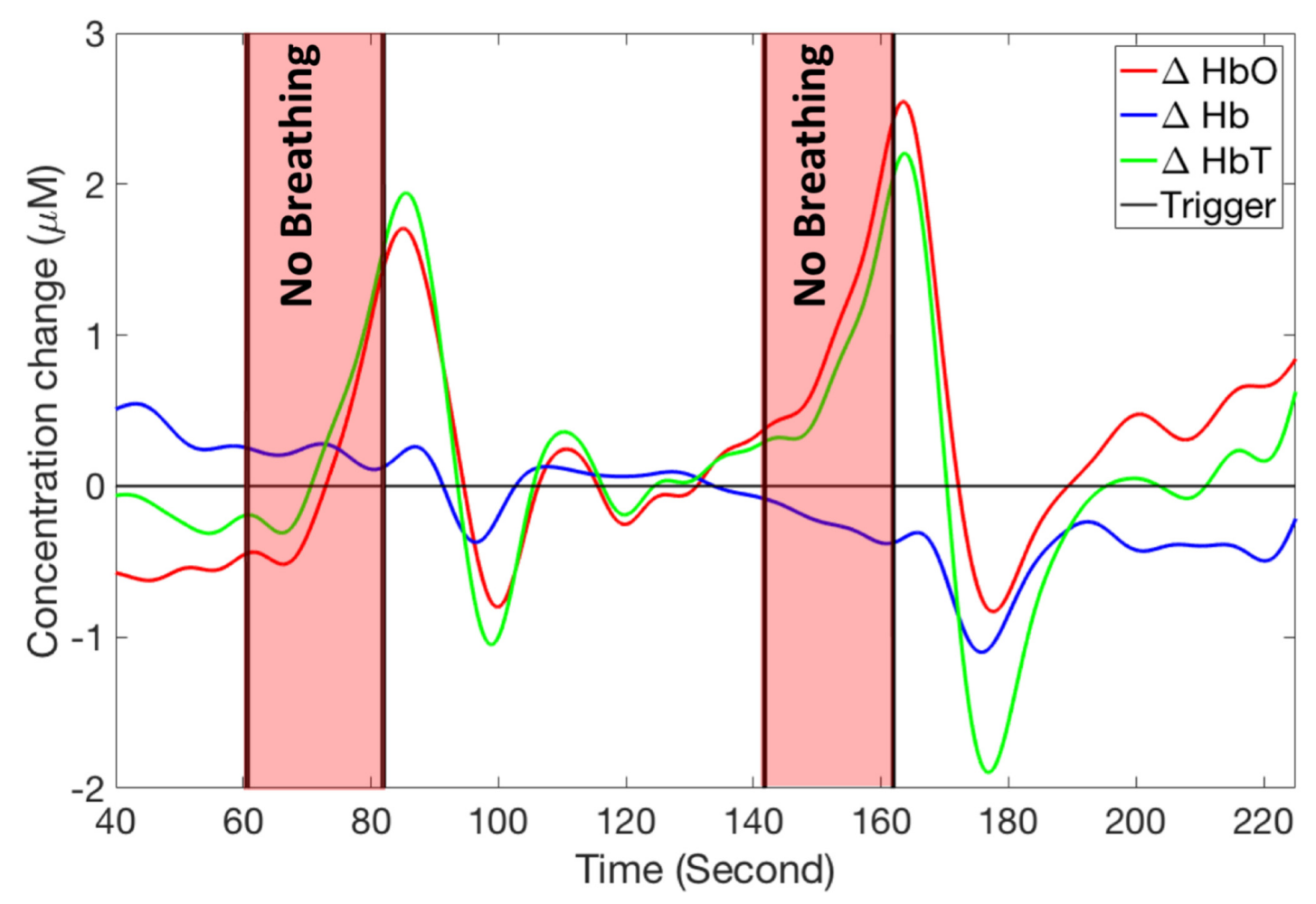

Figure 13. Hemodynamic response derived from the experimental fNIRS data channel shown in the Figure 12.

the second stop and start breathing period respectively.

We have calculated relative changes in oxy-hemoglobin $\left(\Delta \mathrm{HbO}_{2}\right)$ and deoxyhemoglobin $(\Delta H b)$ in the prefrontal cortex from all of the raw intensity measurement data channels using our custom MatLab based fNIRS software. Figure 13 presents the hemodynamic response in the prefrontal cortex computed from a raw experimental fNIRS data channel shown in Figure 12, in response to the breathing exercise over time. We have observed that the concentration of the $\mathrm{HbO}_{2}$ is slowly increased in the two no breath periods. This reveals the low oxygen consumption in the brain in the no no breath periods after a relaxed deep breathing period. The concentration of the $\mathrm{HbO}_{2}$ slowly settled down to the baseline after the start breathing periods. When a normal person performs physical exercises, carbon dioxide $\left(\mathrm{CO}_{2}\right)$ in the blood increases. As a result, heart rate goes up to supply 


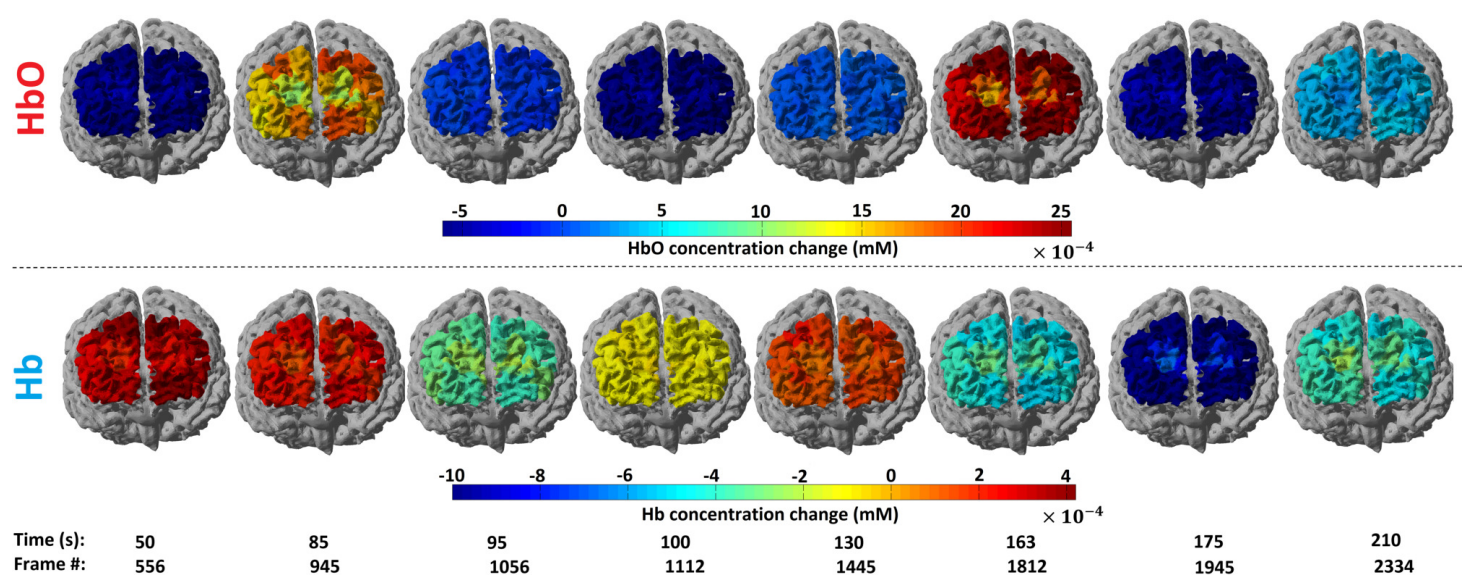

Figure 14. Topographic map of $\mathrm{HbO}_{2}$ and $\mathrm{Hb}$ concentration changes on a 3D cortical surface of the brain at different important time frames from the experimental fNIRS data collected from a participant during the breathing exercise using our WearLight fNIRS system.

more oxygen $\left(\mathrm{O}_{2}\right)$ in the blood. However, in our experiment during the meditative breathing state, our mind and body relaxed more and relaxed muscles no longer needed as much $\mathrm{O}_{2}$. As a result, the $\mathrm{CO}_{2}$ level decreased. During this state, a no breath period further relaxed the participants resulting in reduced heart rate and a positive $\Delta \mathrm{HbO}_{2}$ change in the brain $[64,65,66,67]$. We have also observed that overall reduction of $H b$ concentration in the course of breathing exercise and two dips of $H b$ after the beginning of start breathing periods when the participant breathed rapidly in a high BPM.

The fNIRS imaging shows how relative changes of hemoglobin concentration and hemodynamic responses vary with the subject and the experimental conditions. The maximum total hemoglobin change (Max. $\triangle H b T$ ), and the estimated maximum and minimum heart-rate (Max. BPM and Min. BPM) in this experiment (Exp. 2) for the eight subjects are presented in the Table 4.

We have also topographically mapped $\mathrm{HbO}_{2}$ and $\mathrm{Hb}$ concentration changes on a 3D cortical brain surface offline combining all the fNIRS channels that cover the prefrontal region of the brain. Figure 14 presents the topographic image of 
Table 2. Total hemoglobin concentration change $(\Delta H b T)$ in the forearm occlusion experiment (Exp. 1), and $\triangle H b T$ and BPM changes in breathing experiment (Exp. 2) for the eight subjects.

\begin{tabular}{|c|c|c|c|c|}
\hline $\begin{array}{c}\text { Participant } \\
\text { No. }\end{array}$ & $\begin{array}{c}\text { Exp. 1 } \\
\text { Max. } \Delta H b T \\
(\mu M)\end{array}$ & $\begin{array}{c}\text { Exp. 2 } \\
\text { Max. } \Delta H b T \\
(\mu M)\end{array}$ & $\begin{array}{c}\text { Exp. 2 } \\
\text { Min. } \\
\text { BPM }\end{array}$ & $\begin{array}{c}\text { Exp. 2 } \\
\text { Max. } \\
\text { BPM }\end{array}$ \\
\hline 1 & 45 & 4 & 50 & 75 \\
\hline 3 & 36 & 4.4 & 59 & 81 \\
\hline 3 & 46 & 4.9 & 55 & 79 \\
\hline 4 & 30 & 3.1 & 49 & 76 \\
\hline 5 & 32 & 3.8 & 52 & 73 \\
\hline 6 & 33 & 4.2 & 57 & 80 \\
\hline 7 & 54 & 4.5 & 60 & 81 \\
\hline 8 & 29 & 2.8 & 51 & 74 \\
\hline
\end{tabular}

hemodynamic brain activities on the prefrontal cortex of a participant during the experiment. The $\mathrm{HbO}_{2}$ and $\mathrm{Hb}$ map at different important time frames are shown in order to visualize brain activity in response to the breathing exercise.

\subsection{Discussion}

We focused on the design and development of a new generation fNIRS hardware and software interface based on modern embedded system architecture for the wireless wearable brain monitoring. We have also emphasized highly sensitive comfortable optode design that can be mounted in a general-purpose electrode cap to place on the head. We have exploited the 3D printing technology to design the optode holders and a secure hardware enclosure. The system can be worn around the waist to perform experiments to image hemodynamic activities of a brain in a naturalistic environment.

We configure, control, and acquire data remotely in real-time using a host computer. The system was experimentally evaluated with two sets of preliminary 


\begin{tabular}{|c|c|c|c|c|c|c|c|}
\hline 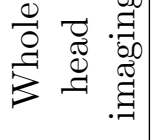 & 운 & 之o & $\stackrel{\circ}{Z}$ & $\sum_{-1}^{0}$ & $\stackrel{\circ}{Z}$ & $\stackrel{\circ}{Z}$ & $\sum_{-1}^{D}$ \\
\hline 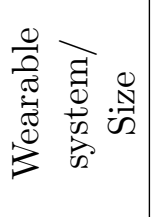 & 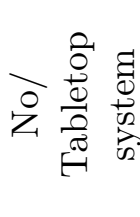 & 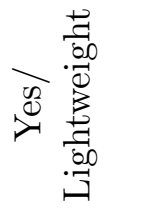 & 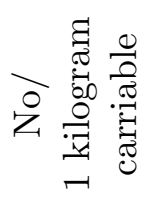 & 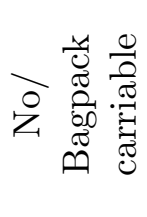 & 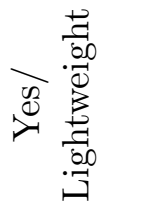 & $\begin{array}{l}\overbrace{}^{\prime} \\
\text { ' }\end{array}$ & 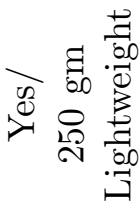 \\
\hline 庖葛 & $\stackrel{\sim}{-}$ & $\stackrel{\sim}{\sim}$ & 1 & $\stackrel{0}{-1}$ & $\stackrel{\mathcal{V}}{\sim}$ & $\stackrel{\sim}{\sim}$ & $\stackrel{\sim}{N}$ \\
\hline 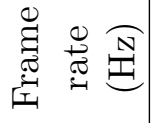 & $\stackrel{\ominus}{-}$ & $\ddot{r}$ & 10 & $\stackrel{\stackrel{2}{\sim}}{0}$ & $\forall$ & 1 & $\exists$ \\
\hline 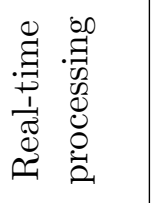 & $\stackrel{\infty}{\infty}$ & 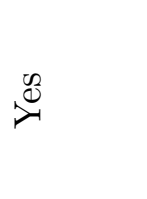 & 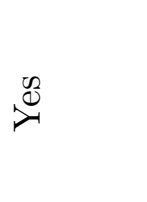 & 1 & 1 & 艺 & $\stackrel{\mathscr{E}}{\nu}$ \\
\hline 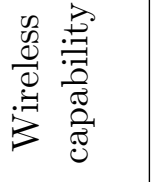 & 之 & 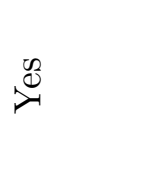 & $\sum_{-1}^{\infty}$ & $\stackrel{\circ}{z}$ & $\underset{d}{\mathscr{C}}$ & 艺 & 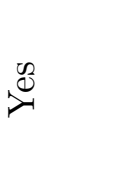 \\
\hline 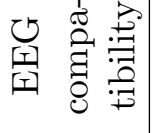 & 之o & 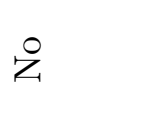 & $\stackrel{0}{Z}$ & $\stackrel{\infty}{\infty}$ & 之o & 兄 & $\stackrel{\mathscr{U}}{\nu}$ \\
\hline 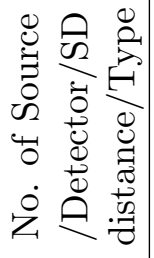 & 글 & 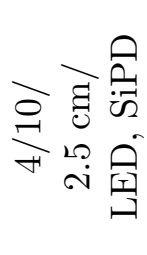 & 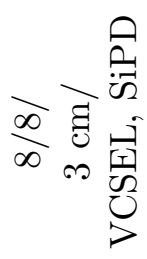 & 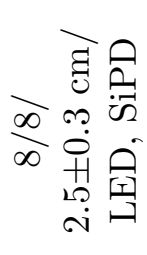 & 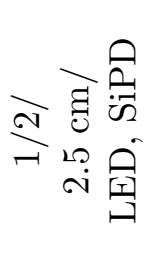 & 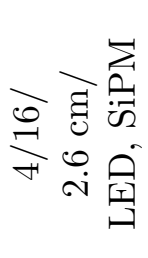 & 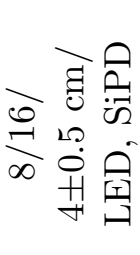 \\
\hline 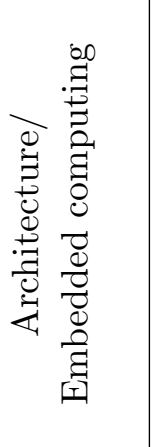 & 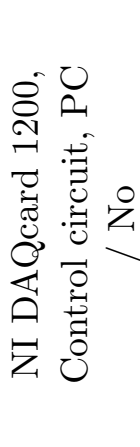 & 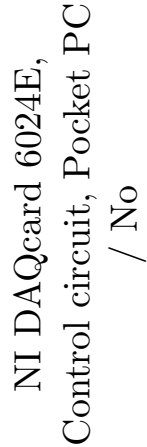 & 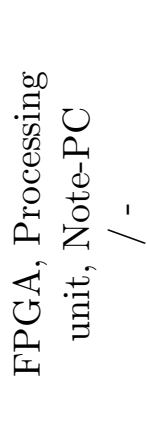 & 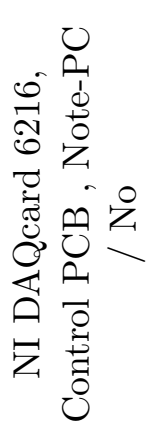 & 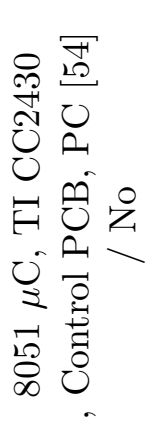 & 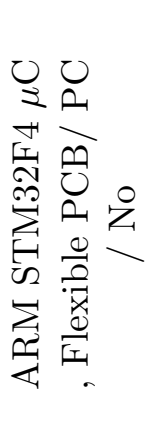 & 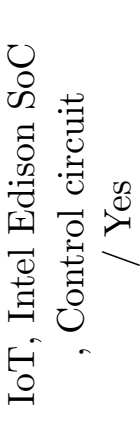 \\
\hline 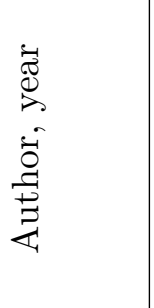 & 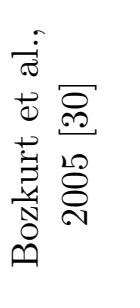 & 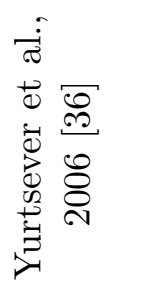 & 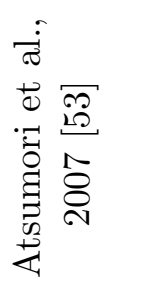 & 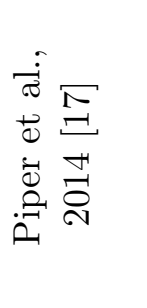 & 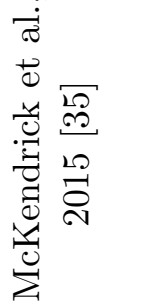 & 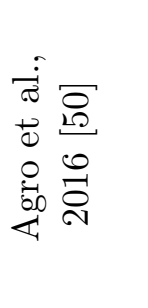 & 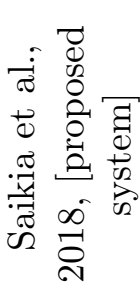 \\
\hline
\end{tabular}


experiments. The hemodynamic response of the muscle tissue in the arterial occlusion experiment, shown in the section 2.5.1, proves that the WearLight fNIRS system works properly. The prefrontal cortex experimental results in section 2.5.2 shows that the system can image hemodynamic activities of the brain. The expected heart rate variation in the breathing exercise also can be observed in the raw experimental data.

There are many challenges in designing a completely configurable batteryoperated multi-channel wearable wireless fNIRS system that can scan the entire brain cortical surface continuously at a high frame rate. The research on portable fNIRS development is growing and some of the innovative fNIRS systems, developed by researchers are mentioned in the section 2.3.2. Table 3 presents the comparison between our proposed system with others. These systems have their own unique advantages and disadvantages. Some of them are not portable enough to carry with the user or they are constrained to operate in a special experimental condition. On the other hand, most of them are not suitable to integrate with EEG electrodes in a general purpose electrode cap to scan the hairy head. Some of fNIRS devices in Table 3 have fewer fNIRS channel, low frame rate, and limited dynamic range. Due to poor sensitivity of the fNIRS channel, they can not be operated with greater SD separations on the hairy head. Though some of the systems are wearable, it is difficult to classify them as a wearable device as they are wired to a computer/laptop via cable or connected to a heavy controlling system. Also, IoT concept based embedded system computing, wireless configurability and complete hardware-GUI software interface were not the focus of their studies for real-time data acquisition, processing and display.

In this work, we have proposed a new generation fNIRS system that has the potential to address some of the common challenges associated with hardware, 
software, configurability, comfort and EEG integration. However, some of the challenges pertaining to the multi-channel wearable fNIRS imaging still remain unaddressed. A single WearLight unit only supports up to 8 dual-wavelength light sources and 16 detectors, and using a single unit, whole brain imaging is not possible. Also, real-time intensive data processing in order to image the brain while participant performing sport was unattempted. However, WearLight allows experiments involving participants performing physical activities. This can advance the fNIRS signal processing domain to find methods for real-time processing of fNIRS data to image a brain in the real world conditions. The preliminary experimental studies evaluate the working of the system. However, we shall perform future studies for more comprehensive brain imaging supported by dedicated experimental protocols. We also desire to integrate EEG with highly sensitive tri-polar concentric ring electrode (TCRE) and our fNIRS system for the multi-modal brain imaging in future studies.

\section{Acknowledgment}

This material is based upon work supported by the National Science Foundation under Grant No. 1539068. The authors would also like to thank Gozde Cay and Dylan Kennedy for their assistance.

\section{List of References}

[1] C. HOCK, K. VILlRINGER, F. MÜLLER-SPAHN, M. HOFMANN, S. SCHUH-HOFER, H. HEEKEREN, R. WENZEL, U. DIRNAGL, and A. VILLRINGER, "Near infrared spectroscopy in the diagnosis of alzheimer's diseasea," Annals of the New York Academy of Sciences, vol. 777, no. 1, pp. 22-29, 1996. [Online]. Available: http://dx.doi.org/10.1111/j.1749-6632. 1996.tb34397.x

[2] F. Irani, S. M. Platek, S. Bunce, A. C. Ruocco, and D. Chute, "Functional near infrared spectroscopy (fnirs): An emerging neuroimaging technology with important applications for the study of brain disorders," The Clinical 
Neuropsychologist, vol. 21, no. 1, pp. 9-37, 2007, pMID: 17366276. [Online]. Available: https://doi.org/10.1080/13854040600910018

[3] A.-C. Ehlis, S. Schneider, T. Dresler, and A. J. Fallgatter, "Application of functional near-infrared spectroscopy in psychiatry," NeuroImage, vol. 85, pp. 478 - 488, 2014, celebrating 20 Years of Functional Near Infrared Spectroscopy (fNIRS). [Online]. Available: http://www.sciencedirect.com/ science/article/pii/S1053811913003200

[4] V. Quaresima and M. Ferrari, "Functional near-infrared spectroscopy (fnirs) for assessing cerebral cortex function during human behavior in natural/social situations: A concise review," Organizational Research Methods, p. 1094428116658959, 2018/01/21 2016. [Online]. Available: https://doi.org/10.1177/1094428116658959

[5] V. Kumar, V. Shivakumar, H. Chhabra, A. Bose, G. Venkatasubramanian, and B. N. Gangadhar, "Functional near infra-red spectroscopy (fnirs) in schizophrenia: A review," Asian Journal of Psychiatry, vol. 27, pp. 18 - 31, 2017. [Online]. Available: http://www.sciencedirect.com/science/article/pii/ S1876201816304348

[6] N. K. Logothetis, "What we can do and what we cannot do with fmri," Nature, vol. 453, no. 7197, pp. 869-878, 2008.

[7] M. A. Yücel, J. J. Selb, T. J. Huppert, M. A. Franceschini, and D. A. Boas, "Functional near infrared spectroscopy: Enabling routine functional brain imaging," Current Opinion in Biomedical Engineering, vol. 4, no. Supplement C, pp. 78 - 86, 2017. [Online]. Available: http://www.sciencedirect.com/science/article/pii/S2468451117300697

[8] G. Strangman, J. P. Culver, J. H. Thompson, and D. A. Boas, "A quantitative comparison of simultaneous bold fmri and nirs recordings during functional brain activation," NeuroImage, vol. 17, no. 2, pp. 719 - 731, 2002. [Online]. Available: http://www.sciencedirect.com/science/article/pii/ S1053811902912279

[9] J. A. Noah, Y. Ono, Y. Nomoto, S. Shimada, A. Tachibana, X. Zhang, S. Bronner, and J. Hirsch, "fmri validation of fnirs measurements during a naturalistic task," Journal of Visualized Experiments : JoVE, no. 100, p. 52116, 2015. [Online]. Available: http://www.ncbi.nlm.nih.gov/pmc/articles/ PMC4544944/

[10] F. F. Jöbsis, "Noninvasive, infrared monitoring of cerebral and myocardial oxygen sufficiency and circulatory parameters," Science, vol. 198, no. 4323, pp. 1264-1267, 1977. [Online]. Available: http://www.jstor.org/stable/1745848 
[11] M. Firbank, E. Okada, and D. T. Delpy, "A theoretical study of the signal contribution of regions of the adult head to near-infrared spectroscopy studies of visual evoked responses," NeuroImage, vol. 8, no. 1, pp. $69-78$, 1998. [Online]. Available: http://www.sciencedirect.com/science/article/pii/ S1053811998903482

[12] M. Ferrari and V. Quaresima, "A brief review on the history of human functional near-infrared spectroscopy (fnirs) development and fields of application," NeuroImage, vol. 63, no. 2, pp. 921 - 935, 2012. [Online]. Available: http://www.sciencedirect.com/science/article/pii/ S1053811912003308

[13] N. Naseer and K.-S. Hong, "fnirs-based brain-computer interfaces: a review," Frontiers in Human Neuroscience, vol. 9, p. 3, 2015. [Online]. Available: http://journal.frontiersin.org/article/10.3389/fnhum.2015.00003

[14] A. T. Eggebrecht, S. L. Ferradal, A. Robichaux-Viehoever, M. S. Hassanpour, H. Dehghani, A. Z. Snyder, T. Hershey, and J. P. Culver, "Mapping distributed brain function and networks with diffuse optical tomography," Nature Photonics, vol. 8, pp. 448 EP -, 05 2014. [Online]. Available: http://dx.doi.org/10.1038/nphoton.2014.107

[15] M. A. Franceschini, V. Toronov, M. E. Filiaci, E. Gratton, and S. Fantini, "On-line optical imaging of the human brain with 160-ms temporal resolution," Opt. Express, vol. 6, no. 3, pp. 49-57, Jan 2000. [Online]. Available: http://www.opticsexpress.org/abstract.cfm?URI=oe-6-3-49

[16] M. M. Ravicz, K. L. Perdue, A. Westerlund, R. E. Vanderwert, and C. A. Nelson, "Infants' neural responses to facial emotion in the prefrontal cortex are correlated with temperament: a functional near-infrared spectroscopy study," Frontiers in Psychology, vol. 6, p. 922, 2015. [Online]. Available: https://www.frontiersin.org/article/10.3389/fpsyg.2015.00922

[17] S. K. Piper, A. Krueger, S. P. Koch, J. Mehnert, C. Habermehl, J. Steinbrink, H. Obrig, and C. H. Schmitz, "A wearable multichannel fnirs system for brain imaging in freely moving subjects," NeuroImage, vol. 85, pp. 64 - 71, 2014, celebrating 20 Years of Functional Near Infrared Spectroscopy (fNIRS). [Online]. Available: http://www.sciencedirect.com/science/article/pii/S1053811913007003

[18] S. Ahn and S. C. Jun, "Multi-modal integration of eeg-fnirs for brain-computer interfaces - current limitations and future directions," Frontiers in Human Neuroscience, vol. 11, p. 503, 2017.

[19] F. Wallois, M. Mahmoudzadeh, A. Patil, and R. Grebe, "Usefulness of simultaneous eeg-nirs recording in language studies," Brain and 
Language, vol. 121, no. 2, pp. 110 - 123, 2012, functional NearInfra Red Spectroscopy (fNIRS): A Promising Functional Imaging Technique for the Study of Brain and Language. [Online]. Available: http://www.sciencedirect.com/science/article/pii/S0093934X11000599

[20] D. K. Nguyen, J. Tremblay, P. Pouliot, P. Vannasing, O. Florea, L. Carmant, F. Lepore, M. Sawan, F. Lesage, and M. Lassonde, "Non-invasive continuous eeg-fnirs recording of temporal lobe seizures," Epilepsy Research, vol. 99, no. 1, pp. 112 - 126, 2012. [Online]. Available: http://www.sciencedirect.com/science/article/pii/S0920121111003627

[21] B. Chance, Z. Zhuang, C. UnAh, C. Alter, and L. Lipton, "Cognitionactivated low-frequency modulation of light absorption in human brain." Proceedings of the National Academy of Sciences of the United States of America, vol. 90, no. 8, pp. 3770-3774, 04 1993. [Online]. Available: http://www.ncbi.nlm.nih.gov/pmc/articles/PMC46383/

[22] A. Yodh and B. Chance, "Spectroscopy and imaging with diffusing light," Physics Today, vol. 48, no. 3, pp. 34-41, 1995.

[23] M. Cope, "The development of a near infrared spectroscopy system and its application for non invasive monitoring of cerebral blood and tissue oxygenation in the newborn infants," Ph.D. dissertation, University of London, 1991.

[24] "Optical absorption of hemoglobin." [Online]. Available: http://omlc.ogi. edu/spectra/

[25] H. Dehghani, B. R. White, B. W. Zeff, A. Tizzard, and J. P. Culver, "Depth sensitivity and image reconstruction analysis of dense imaging arrays for mapping brain function with diffuse optical tomography," Appl. Opt., vol. 48, no. 10, pp. D137-D143, Apr 2009.

[26] M. J. Saikia, R. Kanhirodan, and R. Mohan Vasu, "High-speed gpu-based fully three-dimensional diffuse optical tomographic system," International Journal of Biomedical Imaging, vol. 2014, no. 376456, p. 13, 2014. [Online]. Available: http://dx.doi.org/10.1155/2014/376456

[27] M. J. Saikia and R. Kanhirodan, "Region-of-interest diffuse optical tomography system," Review of Scientific Instruments, vol. 87, no. 1, p. 013701, 2016. [Online]. Available: https://doi.org/10.1063/1.4939054

[28] M. J. Saikia, R. Manjappa, K. Mankodiya, and R. Kanhirodan, "Depth sensitivity improvement of region-of-interest diffuse optical tomography from superficial signal regression," in Imaging and Applied Optics 2018 (3D, AO, AIO, COSI, DH, IS, LACSEA, LSEC, MATH, pcAOP). Optical Society of America, 2018, p. CM3E.5. [Online]. Available: http://www.osapublishing.org/abstract.cfm?URI=COSI-2018-CM3E.5 
[29] S. M. Liao, S. L. Ferradal, B. R. White, N. Gregg, T. E. Inder, and J. P. Culver, "High-density diffuse optical tomography of term infant visual cortex in the nursery," Journal of Biomedical Optics, vol. 17, no. 8, p. 081414, 08 2012. [Online]. Available: http: //www.ncbi.nlm.nih.gov/pmc/articles/PMC3391961/

[30] A. Bozkurt, A. Rosen, H. Rosen, and B. Onaral, "A portable near infrared spectroscopy system for bedside monitoring of newborn brain," BioMedical Engineering OnLine, vol. 4, no. 1, p. 29, Apr 2005. [Online]. Available: https://doi.org/10.1186/1475-925X-4-29

[31] B. R. White and J. P. Culver, "Phase-encoded retinotopy as an evaluation of diffuse optical neuroimaging," NeuroImage, vol. 49, no. 1, pp. $568-577$, 2010. [Online]. Available: http://www.sciencedirect.com/science/article/pii/ S1053811909007939

[32] M. J. Saikia, R. Manjappa, and R. Kanhirodan, "A cost-effective led and photodetector based fast direct $3 \mathrm{~d}$ diffuse optical imaging system," in Diffuse Optical Spectroscopy and Imaging VI. Optical Society of America, 2017, p. 104120Y. [Online]. Available: http: //www.osapublishing.org/abstract.cfm?URI=ECBO-2017-104120Y

[33] M. J. Saikia, R. Kanhirodan, and R. M. Vasu, "High-speed gpu-based fully three-dimensional diffuse optical tomographic system," Journal of Biomedical Imaging, vol. 2014, pp. 3:3-3:3, Jan. 2014. [Online]. Available: http://dx.doi.org/10.1155/2014/376456

[34] S. M. Coyle, T. E. Ward, and C. M. Markham, "Brain-computer interface using a simplified functional near-infrared spectroscopy system," Journal of Neural Engineering, vol. 4, no. 3, p. 219, 2007. [Online]. Available: http://stacks.iop.org/1741-2552/4/i=3/a $=007$

[35] R. McKendrick, R. Parasuraman, and H. Ayaz, "Wearable functional near infrared spectroscopy (fnirs) and transcranial direct current stimulation (tdcs): expanding vistas for neurocognitive augmentation," Frontiers in Systems Neuroscience, vol. 9, p. 27, 2015. [Online]. Available: http://journal.frontiersin.org/article/10.3389/fnsys.2015.00027

[36] G. Yurtsever, F. Kepics, and A. Bozkurt, "Wireless continuous wave near infrared imager," in 2003 IEEE 29th Annual Proceedings of Bioengineering Conference, March 2003, pp. 53-54.

[37] M. Abtahi, G. Cay, M. J. Saikia, and K. Mankodiya, "Designing and testing a wearable, wireless fnirs patch," in 2016 38th Annual International Conference of the IEEE Engineering in Medicine and Biology Society (EMBC), Aug 2016, pp. 6298-6301. 
[38] A. Villringer and B. Chance, "Non-invasive optical spectroscopy and imaging of human brain function," Trends in Neurosciences, vol. 20, no. 10, pp. 435 - 442, 1997. [Online]. Available: http://www.sciencedirect.com/science/ article/pii/S0166223697011326

[39] T. J. Huppert, S. G. Diamond, M. A. Franceschini, and D. A. Boas, "Homer: a review of time-series analysis methods for near-infrared spectroscopy of the brain," Appl. Opt., vol. 48, no. 10, pp. D280-D298, Apr 2009. [Online]. Available: http://ao.osa.org/abstract.cfm?URI=ao-48-10-D280

[40] L. Kocsis, P. Herman, and A. Eke, "The modified beer-lambert law revisited," Physics in Medicine \& Biology, vol. 51, no. 5, p. N91, 2006. [Online]. Available: http://stacks.iop.org/0031-9155/51/i=5/a=N02

[41] D. T. Delpy, M. Cope, P. van der Zee, S. Arridge, S. Wray, and J. Wyatt, "Estimation of optical pathlength through tissue from direct time of flight measurement," Physics in Medicine \& Biology, vol. 33, no. 12, p. 1433, 1988. [Online]. Available: http://stacks.iop.org/0031-9155/33/i=12/a=008

[42] S. Brigadoi, L. Ceccherini, S. Cutini, F. Scarpa, P. Scatturin, J. Selb, L. Gagnon, D. A. Boas, and R. J. Cooper, "Motion artifacts in functional near-infrared spectroscopy: A comparison of motion correction techniques applied to real cognitive data," NeuroImage, vol. 85, pp. 181 - 191, 2014, celebrating 20 Years of Functional Near Infrared Spectroscopy (fNIRS). [Online]. Available: http://www.sciencedirect.com/science/article/ pii/S1053811913004291

[43] M. Sood, P. Besson, M. Muthalib, U. Jindal, S. Perrey, A. Dutta, and M. Hayashibe, "Nirs-eeg joint imaging during transcranial direct current stimulation: Online parameter estimation with an autoregressive model," Journal of Neuroscience Methods, vol. 274, pp. 71 - 80, 2016.

[44] G. Gallegos-Ayala, A. Furdea, K. Takano, C. A. Ruf, H. Flor, and N. Birbaumer, "Brain communication in a completely locked-in patient using bedside near-infrared spectroscopy," Neurology, vol. 82, no. 21, pp. 1930-1932, 2014. [Online]. Available: http://n.neurology.org/content/82/21/1930

[45] U. Chaudhary, N. Birbaumer, and A. Ramos-Murguialday, "Braincomputer interfaces for communication and rehabilitation," Nature Reviews Neurology, vol. 12, pp. 513 EP -, 08 2016. [Online]. Available: http://dx.doi.org/10.1038/nrneurol.2016.113

[46] G. Kaimal, H. Ayaz, J. Herres, R. Dieterich-Hartwell, B. Makwana, D. H. Kaiser, and J. A. Nasser, "Functional near-infrared spectroscopy assessment of reward perception based on visual self-expression: Coloring, doodling, and free drawing," The Arts in Psychotherapy, vol. 55, pp. 85 - 92, 
2017. [Online]. Available: http://www.sciencedirect.com/science/article/pii/ S019745561630171X

[47] M. Izzetoglu, K. Izzetoglu, S. Bunce, H. Ayaz, A. Devaraj, B. Onaral, and K. Pourrezaei, "Functional near-infrared neuroimaging," IEEE Transactions on Neural Systems and Rehabilitation Engineering, vol. 13, no. 2, pp. 153-159, 2005.

[48] E. Kamrani, F. Lesage, and M. Sawan, "Fully on-chip integrated photodetector front-end dedicated to real-time portable optical brain imaging," Optics and Photonics Journal, vol. Vol.02No.04, p. 13, 2012. [Online]. Available: //www.scirp.org/journal/PaperInformation.aspx? PaperID $=25968 \%] 25968$

[49] D. Chitnis, D. Airantzis, D. Highton, R. Williams, P. Phan, V. Giagka, S. Powell, R. J. Cooper, I. Tachtsidis, M. Smith, C. E. Elwell, J. C. Hebden, and N. Everdell, "Towards a wearable near infrared spectroscopic probe for monitoring concentrations of multiple chromophores in biological tissue in vivo," Review of Scientific Instruments, vol. 87, no. 6, p. 065112, 2016. [Online]. Available: https://doi.org/10.1063/1.4954722

[50] D. Agrò, R. Canicattì, M. Pinto, G. Morsellino, A. Tomasino, G. Adamo, L. Curcio, A. Parisi, S. Stivala, N. Galioto, et al., "Design and implementation of a portable fnirs embedded system," in Applications in Electronics Pervading Industry, Environment and Society. Springer, 2016, pp. 43-50.

[51] A. von Lühmann, H. Wabnitz, T. Sander, and K. R. Müller, "M3ba: A mobile, modular, multimodal biosignal acquisition architecture for miniaturized eeg-nirs-based hybrid bci and monitoring," IEEE Transactions on Biomedical Engineering, vol. 64, no. 6, pp. 1199-1210, 2017.

[52] M. J. Saikia and R. Kanhirodan, "Development of dot system for roi scanning," in 12th International Conference on Fiber Optics and Photonics. Optical Society of America, 2014, p. T3A.4. [Online]. Available: http://www.osapublishing.org/abstract.cfm?URI=Photonics-2014-T3A.4

[53] H. Atsumori, M. Kiguchi, A. Obata, H. Sato, T. Katura, K. Utsugi, T. Funane, and A. Maki, "Development of a multi-channel, portable optical topography system," in 2007 29th Annual International Conference of the IEEE Engineering in Medicine and Biology Society, 2007, pp. 3362-3364.

[54] H. Ayaz, B. Onaral, K. Izzetoglu, P. A. Shewokis, R. McKendrick, and R. Parasuraman, "Continuous monitoring of brain dynamics with functional near infrared spectroscopy as a tool for neuroergonomic research: empirical examples and a technological development," Frontiers in Human Neuroscience, vol. 7, p. 871, 2013. [Online]. Available: http://www.ncbi.nlm.nih.gov/pmc/articles/PMC3866520/ 
[55] A. Bozkurt and B. Onaral, "Safety assessment of near infrared light emitting diodes for diffuse optical measurements," BioMedical Engineering OnLine, vol. 3, pp. $9-9,2004$.

[56] R. Cooper, J. Selb, L. Gagnon, D. Phillip, H. Schytz, H. Iversen, M. Ashina, and D. Boas, "A systematic comparison of motion artifact correction techniques for functional near-infrared spectroscopy," Frontiers in Neuroscience, vol. 6, p. 147, 2012. [Online]. Available: https: //www.frontiersin.org/article/10.3389/fnins.2012.00147

[57] Y. Zhang, D. H. Brooks, M. A. Franceschini, and D. A. Boas, "Eigenvectorbased spatial filtering for reduction of physiological interference in diffuse optical imaging," Journal of biomedical optics, vol. 10, no. 1, p. 011014, 2005.

[58] H. Santosa, M. Jiyoun Hong, S.-P. Kim, and K.-S. Hong, "Noise reduction in functional near-infrared spectroscopy signals by independent component analysis," Review of Scientific Instruments, vol. 84, no. 7, p. 073106, 2013. [Online]. Available: https://doi.org/10.1063/1.4812785

[59] M. Izzetoglu, P. Chitrapu, S. Bunce, and B. Onaral, "Motion artifact cancellation in nir spectroscopy using discrete kalman filtering," BioMedical Engineering OnLine, vol. 9, no. 1, p. 16, 2010. [Online]. Available: https://doi.org/10.1186/1475-925X-9-16

[60] X. Cui, S. Bray, and A. L. Reiss, "Functional near infrared spectroscopy (nirs) signal improvement based on negative correlation between oxygenated and deoxygenated hemoglobin dynamics," NeuroImage, vol. 49, no. 4, pp. 3039 - 3046, 2010. [Online]. Available: http://www.sciencedirect.com/ science/article/pii/S105381190901235X

[61] B. Molavi and G. A. Dumont, "Wavelet-based motion artifact removal for functional near-infrared spectroscopy," Physiological Measurement, vol. 33, no. 2, p. 259, 2012. [Online]. Available: http://stacks.iop.org/0967-3334/33/ $\mathrm{i}=2 / \mathrm{a}=259$

[62] F. Scholkmann, S. Spichtig, T. Muehlemann, and M. Wolf, "How to detect and reduce movement artifacts in near-infrared imaging using moving standard deviation and spline interpolation," Physiological Measurement, vol. 31, no. 5, p. 649, 2010. [Online]. Available: http: //stacks.iop.org/0967-3334/31/i=5/a=004

[63] Y. Shang, Y. Zhao, R. Cheng, L. Dong, D. Irwin, and G. Yu, "Portable optical tissue flow oximeter based on diffuse correlation spectroscopy," Opt. Lett., vol. 34, no. 22, pp. 3556-3558, Nov 2009. [Online]. Available: http://ol.osa.org/abstract.cfm?URI=ol-34-22-3556 
[64] K. Singh, H. Bhargav, and T. Srinivasan, "Effect of uninostril yoga breathing on brain hemodynamics: A functional near-infrared spectroscopy study," International Journal of Yoga, vol. 9, no. 1, pp. 12-19, Jan-Jun 2016. [Online]. Available: http://www.ncbi.nlm.nih.gov/pmc/articles/PMC4728953/

[65] S. Deepeshwar, S. A. Vinchurkar, N. K. Visweswaraiah, and H. R. Nagendra, "Hemodynamic responses on prefrontal cortex related to meditation and attentional task," Frontiers in Systems Neuroscience, vol. 8, p. 252, 2015. [Online]. Available: https://www.frontiersin.org/article/10.3389/fnsys.2014. 00252

[66] R. B. Ankad, A. Herur, S. Patil, G. Shashikala, and S. Chinagudi, "Effect of short-term pranayama and meditation on cardiovascular functions in healthy individuals," Heart Views : The Official Journal of the Gulf Heart Association, vol. 12, no. 2, pp. 58-62, Apr-Jun 2011. [Online]. Available: http://www.ncbi.nlm.nih.gov/pmc/articles/PMC3221193/

[67] "Yogasynergy," $2009 . \quad$ [Online]. Available: https://yogasynergy.com/2010/08/10/ how-to-slow-the-heart-from-88-beats-to-32-beat-per-minute-in- 45 -seconds / 


\section{MANUSCRIPT 3}

\section{A configurable wireless optical brain monitor based on internet-of-things services}

by

Manob Jyoti Saikia, Gozde Cay, Joshua V. Gyllinsky, Kunal Mankodiya

published in

IEEE International Conference on Electronics, Communication, Computer Technologies and Optimization Techniques (ICEECCOT-2018), Mysuru, India, Dec. 14-15, 2018. 


\subsection{Abstract}

There is an increasing interest in understanding the brain in its natural state. This recently led to the wide adoption of portable brain monitors including functional Near-Infrared Spectroscopy (fNIRS) which uses near-infrared (NIR) light to capture the brain's hemodynamic responses, also known as cerebral oxygenation. Nowadays, fNIRS is used in clinical settings of various medical fields including neurology, neuroscience, clinical psychology, and psychiatry. Compared to its counterpart, functional Magnetic Resonance Imaging (fMRI), fNIRS is portable and provides higher temporal resolution for scanning the hemodynamic response of the cortical layer of the brain. fNIRS has the potential to work as a smart wearable device to monitor a brain in an unconstrained setting. However, wearable implementation is challenging as the fNIRS system demands a complex circuit to drive NIR light sources and detect low intensity reflected light from the scalp. Also, the algorithm for the real-time controlling, configuration, data acquisition, and signal processing are complex for a wearable smart fNIRS. Currently available fNIRS systems are not intelligent, completely wearable or designed as a smart body sensor network (BSN) node. In this paper, we present our recent design of a configurable fNIRS system that acts as a BSN node controlled by a remote Graphical User Interface (GUI). The presented fNIRS system is based on an embedded board where we deployed a internet-of-things (IoT) based service-oriented architecture providing the functionality of montage configuration, fNIRS signal testing, calibration, channel selection, data collection and wireless data transmission to the GUI. This development can improve the flexibility of the fNIRS system regarding its use in natural environments where the brain functions of moving human subjects are monitored. We have experimentally evaluated working of the hardware, software, GUI and wireless data transmission, and capability of detecting hemodynamic 
responses.

\subsection{Introduction}

Portable brain monitoring is widely adopted in the research environment to study the human brain under various natural conditions in recent years. For example, there is an increasing interest in research communities to learn more about the functions of the brain when the human is playing a sport, playing a musical instrument or interacting in a social situation $[1,2]$. Brain motor activations are also of interest when the body is in motion or performing exercises involving upper and lower limbs [3]. Such brain studies are not possible using the conventional neuroimaging technologies, such as magnetic resonance imaging (MRI) and computed tomography $(\mathrm{CT})$ which require the body to be in a motionless state. Therefore, brain monitoring systems electroencephalography (EEG) and functional near-infrared spectroscopy (fNIRS) are increasingly used for brain studies that involve participants are in their natural states or performing physical activities, because both of these technologies are portable [4]. EEG is a neuroelectric method to monitor brain activity [5] where multiple electrodes on the scalp are placed to capture brain signals. On the other hand, fNIRS is based on the optical method where optodes (light source and detector) are placed on the scalp to capture the hemodynamic response of the brain $[6,7,2,8]$. Studies report that fNIRS is less susceptible to motion artifact and higher spatial resolution compared its counterpart, EEG [9].

fNIRS brain imaging systems use multiple near-infrared (NIR) light sources in the wavelength range from $600 \mathrm{~nm}$ to $900 \mathrm{~nm}$ and light detectors to place on the scalp in a predefined arrangement, also known as optode montage [10]. The emitted light from an NIR source travels through the tissue, and the detector detects the transmitted light reflected from the cortical surface as shown in Figure 25 (a). The 
(a)

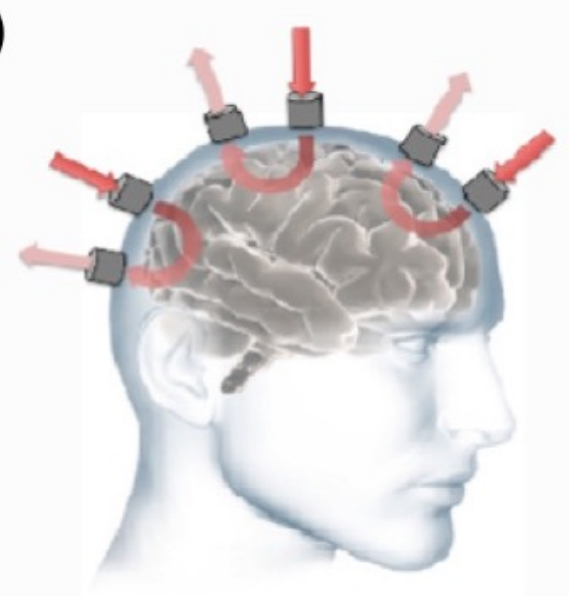

$\sum$ (b)

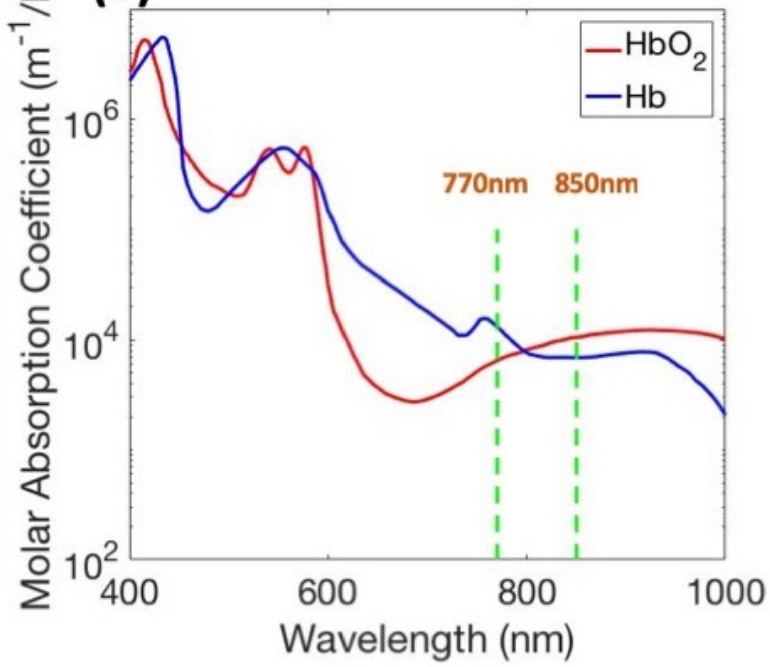

Figure 15. (a) NIR light propagation from the sources to detectors carrying information of the hemodynamic activities of the brain, (b) plot of absorption spectra of $\mathrm{HbO}_{2}$ and $\mathrm{Hb}$ in the NIR window

penetration depth of the NIR light and fNIRS signal strength largely depend on source-detector separations and montage selection $[11,12,13]$. It is also seen that hemodynamic response of the brain also varies with experimental paradigm and participants. For example, some brain studies require monitoring a small region of the brain with high-density optodes [14], while some studies require covering a larger area of the brain[15]. For this reason, it is essential to have the flexibility of online configurability of montage, thereby changing the source-detector arrangement to have an optimal setup during an experiment. Moreover, experiments with different montages supply us the clue about different brain regions. Also, online testing of the other important parameters such as signal-to-noise ratio of the fNIRS channels, the coupling of the optodes on the scalp, ambient light level, and calibration can ensure a quality fNIRS data collection.

Wireless body sensor networks (BSN) can provide promising future in monitoring physiological parameter for the health monitoring and medical diagnosis. Recently wearable implementations of brain monitoring using EEG is being pur- 


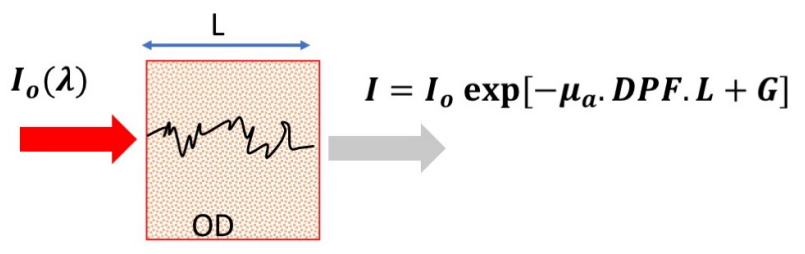

Absorbing+Scattering

medium

Absorption Coefficient $\boldsymbol{\mu}_{\boldsymbol{a}}=\boldsymbol{\varepsilon} \boldsymbol{c}$

Absorption/Attenuation (A) as Optical density

$$
\begin{gathered}
A=O D=\ln \left(I_{0} / I\right)=\varepsilon \cdot C \cdot D P F \cdot L+G \\
\Delta O D(t)=-\ln \left(I(t) / I_{0}(t=0)\right) \\
=\left(\varepsilon_{H b}(\lambda) \cdot \Delta[H b]+\varepsilon_{H b O}(\lambda) \cdot \Delta[H b O]\right) \cdot D P F(\lambda) . L
\end{gathered}
$$

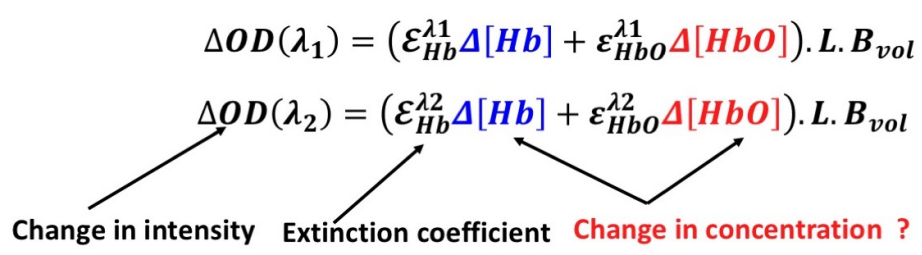

Figure 16. Principles of Modified Beer-Lambert Law.

sued by research and commercial organizations [16]. However, fNIRS as a BSN demands sophisticated wearable implementation of the hardware and reliable integration with the optodes. It also requires an algorithm along with a data processing capability, which can wirelessly operate the fNIRS device with less intervention from the user and power-efficient wireless data transmission to a host device or cloud storage. Another challenge is that fNIRS system as BSN are often susceptible to physiological and environmental noise. Hence, fNIRS requires integration of robust motion artifact and noise removal algorithm [17]. To address these challenges, in this paper we present the development of a configurable fNIRS brain monitor which makes the following scientific contributions:

- A hardware and software architecture for an fNIRS system that works as a wireless body sensor network (BSN) node was proposed. 
- We have created and deployed a IoT based service-oriented architecture for the fNIRS BSN module for montage configuration, calibration, channel selection, SNR testing and data collection.

- A graphical user interface (GUI) was developed to configure and operate the fNIRS system wirelessly from a host computer.

\subsection{Background of fNIRS}

Functional near-infrared spectroscopy is an emerging neuroimaging modality that can non-invasively map the hemodynamic response of the brain by measuring changes in oxygenated $\left(\mathrm{HbO}_{2}\right)$ and deoxygenated $(\mathrm{Hb})$ hemoglobin concentration on the cortical surface of the brain $[6,10]$. NIR light source of two different wavelengths (one wavelength at a time) is used to shine on the scalp and light that propagates through the tissue is absorbed by the chromophores[18, 19, 20]. The back reflected light intensity from the cortical surface of the brain is measured using detectors as shown in Figure 25 (a). The light absorption by the chromophores is wavelength dependent. The absorption spectra of the two major chromophores $\mathrm{HbO}_{2}$ and $\mathrm{Hb}$ in the NIR window is plotted in the Figure 25 (b). Probing with two

distinct wavelengths in the NIR window, can resolve the concentration changes of $\mathrm{HbO}_{2}$ and $\mathrm{Hb}$ on the cortical surface of the brain over time. The hemoglobin concentration changes are calculated using the Modified Beer-Lambert Law (MBLL) [21]. The principle of MBLL is shown in Figure 2.

Photodetectors are used to detect reflected light from brain. To ensure a strong detectability, the distance between the source and detector is important because the optical path length is more than the actual distance between the source and detector and while the distance increases, detectability decreases[11, 22]. The human head has different tissue layers, and all the layers have different scattering and absorption rates, a 3 to $4 \mathrm{~cm}$ distance between the source and detector 
is suitable for a detectable fNIRS signal from brain $[23,24,25]$. In general, an fNIRS system has (1) fNIRS sensors (optodes) made of light source (LED or laser) and photodetector, (2) a control unit which controls the system by switching the light sources and collecting the electrical signal from photodetectors with an controlling algorithm and (3) a computer that collects the raw data, makes necessary computations on it and displays the results.
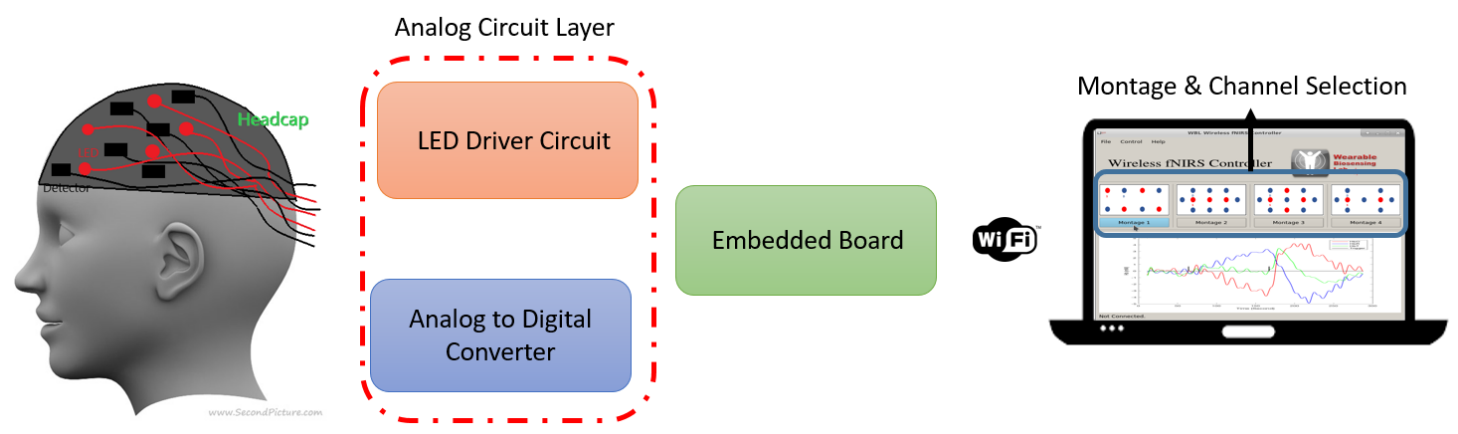

Figure 17. A conceptual diagram of configurable fNIRS system.

\subsubsection{Related Works}

Most publications are on the comparison of fNIRS with EEG and fMRI, and the clinical aspect of the fNIRS system. These studies suggested fNIRS as a better alternative or multi-model brain imaging combining fNIRS with EEG[4, 26, 7]. However, there are some studies available related to the fNIRS hardware development. In a report by Bozkurt et al. [27], a low-cost, battery operated, dualwavelength, continuous wave NIR system for monitoring newborns was presented. As an fNIRS sensor, they used two LEDs and one photodetector and placed them in a flexible circuit. As a control unit, a data acquisition card (DAQ card) 1200 was used and the raw data was sent to a computer for data processing and display. In another study by Yurtsever et al. [28], a wireless NIRS system was developed. For the fNIRS sensor, they used four LEDs and ten photodetectors with a flex- 
ible sensor pad. As control unit, they used DAQ card and pocket PC. The raw data was sent to a computer using a pocket PC. Another study represented by Atsumori et al. [29] showed that they developed a multi-channel, portable optical topography system. They used vertical cavity surface emitting lasers (VCSEL) as light sources. In their sensor, there were eight sources and eight detectors. To process the data, a field programmable gate array (FPGA) was used. The raw signal was sent to a computer either via wireless or flash memory. Recently, Agro et al. [30] offered a portable fNIRS system. As an fNIRS sensor, they used sixty-four LEDs and one hundred twenty-eight silicon photomultiplier (SiPM) optical diodes to cover entire skull area. They placed the optodes into a divided flexible board; each area consists of four LEDs and sixteen SiPMs. As a control unit, an ARM microcontroller based main board was used. The main board connected to the computer via UART/RS232 and UART/USB connection.

\subsection{A Configurable fNIRS System}

We have proposed a configurable fNIRS system that acts as a smart BSN device. We have developed fNIRS hardware, IoT based service-oriented software architecture and fNIRS controlling graphical user interface (GUI) software.

\subsection{1 fNIRS Hardware}

Our fNIRS hardware is composed of embedded board controller, analog circuit, and optode montage as shown in the block diagram in Figure 28.

\section{Embedded Controller}

The embedded board used in our system, Raspberry Pi Zero W coordinates all the tasks including receiving commands from a host user wirelessly, configuring optodes of an experiment, triggering the LEDs and measuring the detected fNIRS signal during the experiment and sending data to the host wirelessly. The com- 


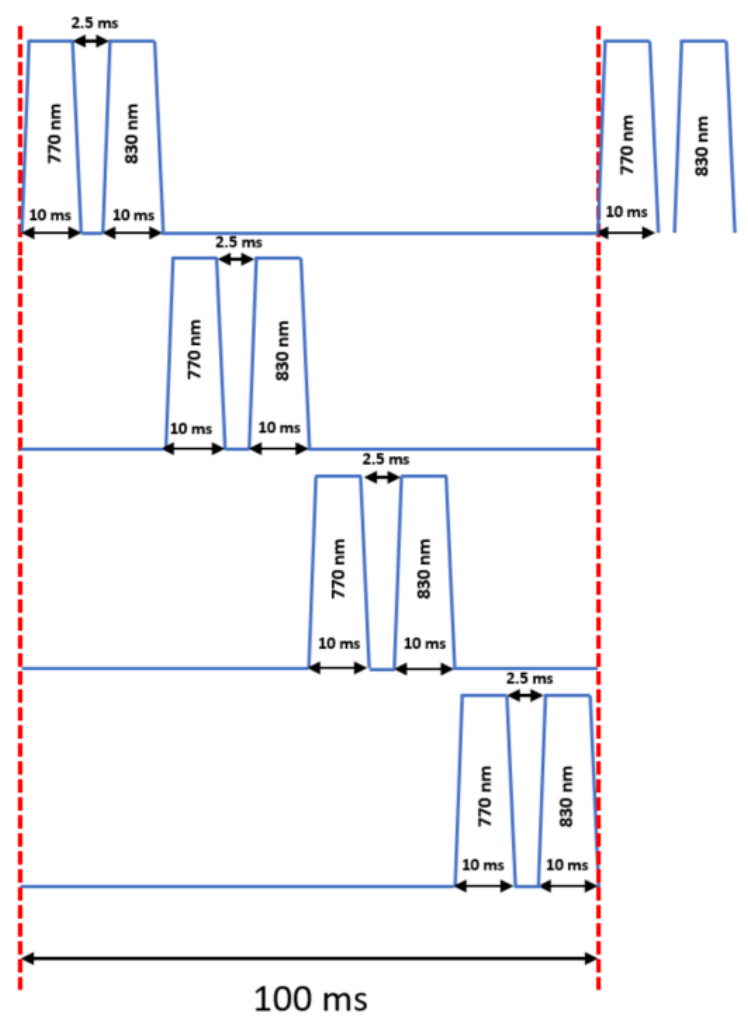

Figure 18. Timing sequence for triggering LEDs.

puter module sends the triggering signal to DEMUX and LED drivers present in the analog circuit layer to drive the LEDs according to a multiplexing algorithm configured by the user. Then it measures the reflected light which is detected by the detector and digitized by the ADC. It sends the fNIRS data to the host computer wirelessly to display the fNIRS signal. Figure 29 illustrates dual-wavelengths LED multiplexing scheme [31] that has been implemented in our system. The LEDs turn on for $10 \mathrm{~ms}$ sequentially and there is a $2.5 \mathrm{~ms}$ OFF time between each LEDs. Thus, the total duration of a cycle is $100 \mathrm{~ms}$.

\section{Analog Circuit Design}

The analog circuit layer was designed to provide constant, yet adjustable current to each of the LEDs. It also provides regulated bias voltages and reference voltages to each of the circuit components and detectors. A 16-bit 4-channel preci- 
sion Analog to Digital Converter (ADC), ADS1115, present in this layer converts analog electrical signal to digital signal. The ADC is controlled by an embedded computing module via $\mathrm{I} 2 \mathrm{C}$ communication protocol. The $\mathrm{ADC}$ readings are transferred to the embedded compute module for the further data conversion, processing and wireless transmission to a computer.

\section{Optode Montage}

The placement of sources and detectors on the scalp with a predefined arrangement is known as optode montage [32]. The location of each optode and the distance between source and detector are very important for collecting reliable data. The human brain has different regions and each region is associated with different human activities. Hence, different optode configuration to measure from a different region of the bran is essential. Figure 38 shows some brain regions and possible montages.

In this design, dual wavelengths LEDs which have wavelengths of $770 \mathrm{~nm}$ and $850 \mathrm{~nm}$ are employed as a source optode and silicon-photodiode detectors as detector optode. The distance between sources and detectors are 3-4 cm. Our montage has four pairs of LED and photodetector that form 10 fNIRS channels, 20 measurement points in total. The optodes are placed on the head using a generalpurpose electrode cap. In this design, four montages are currently supported, but more can be easily added. These optional montages are shown as selectable options [Fig. 22].

\subsubsection{Service oriented architecture on embedded board}

A service-oriented architecture (SOA) is a software design that allows application components to provide services to other components by using a communication protocol [33]. In our system presented in this paper, a software was developed to 


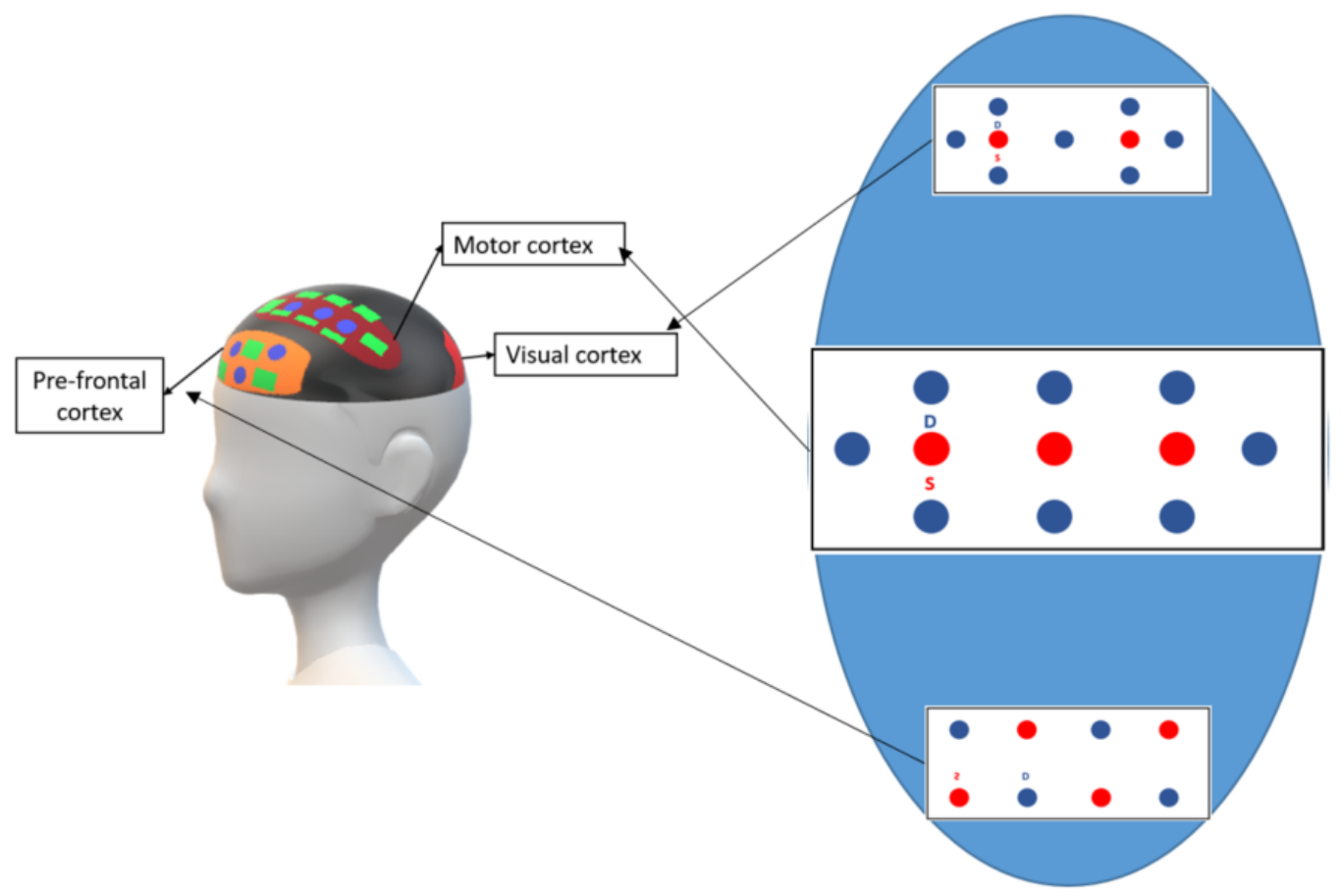

Figure 19. Different regions of the brain and possible optode montages.

provide services to the fNIRS device remotely. These services are:

- Checking the signal quality and the optode placement.

- Montage and channel selection.

- Displaying raw data and plotting.

- Displaying pre-rendered graphics from local storage or transmitted if the fNIRS compute unit supports it.

To check signal quality and optode placement, two threshold values, $V_{t h(\min )}$ and $V_{t h(\max )}$ of voltages which is read from ADC pin were determined through hardware testing and experimental evaluation. We set $V_{t h(\min )}=0.05 \mathrm{v}$ and $V_{t h(\max )}=1.2 \mathrm{v}$ to ensure that the optodes are placed properly on the scalp. For montage selection, Python scripts for different montages were developed and uploaded into the Raspberry Pi. These scripts were designed to be operated over 


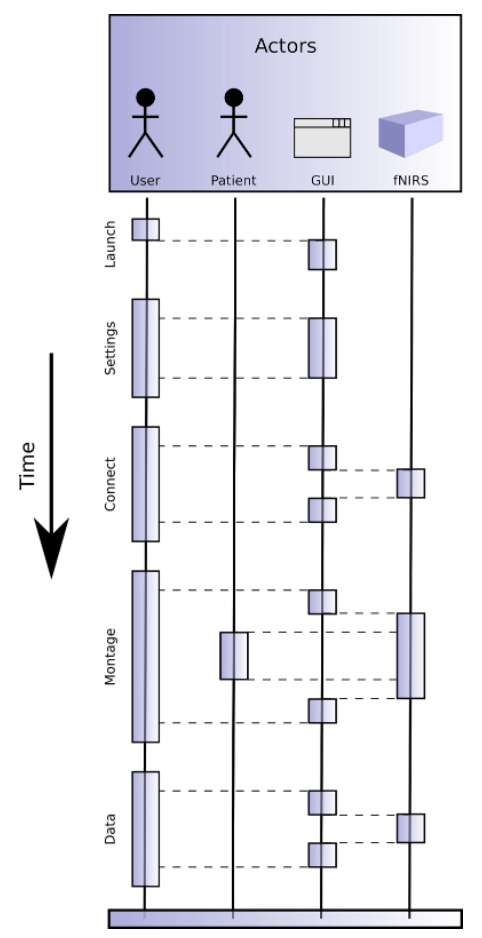

Figure 20. The flowchart of fNIRS application.

terminal control locally and run with userland permission levels. The user can see different montages and make a selection between them. According to the user's selection, a Python code is run remotely. All services are self-contained, they can be run independently and they all have a specific outcome. Furthermore, the participants or end users can run the services without knowing the content of the program, since they are embedded in a remote control. Therefore, our services fulfill the properties of SOA [34].

\subsubsection{Controlling Graphical User Interface (GUI)}

In this study, we developed our system to have remote configurability. To use the fNIRS system remotely, we created a graphical user interface (GUI) which connects to the fNIRS BSN node over the internet. It allows experimenters or end users to change the optode montage from the computer. The GUI contains push buttons and figures of montages. The experimenter can see the montage 

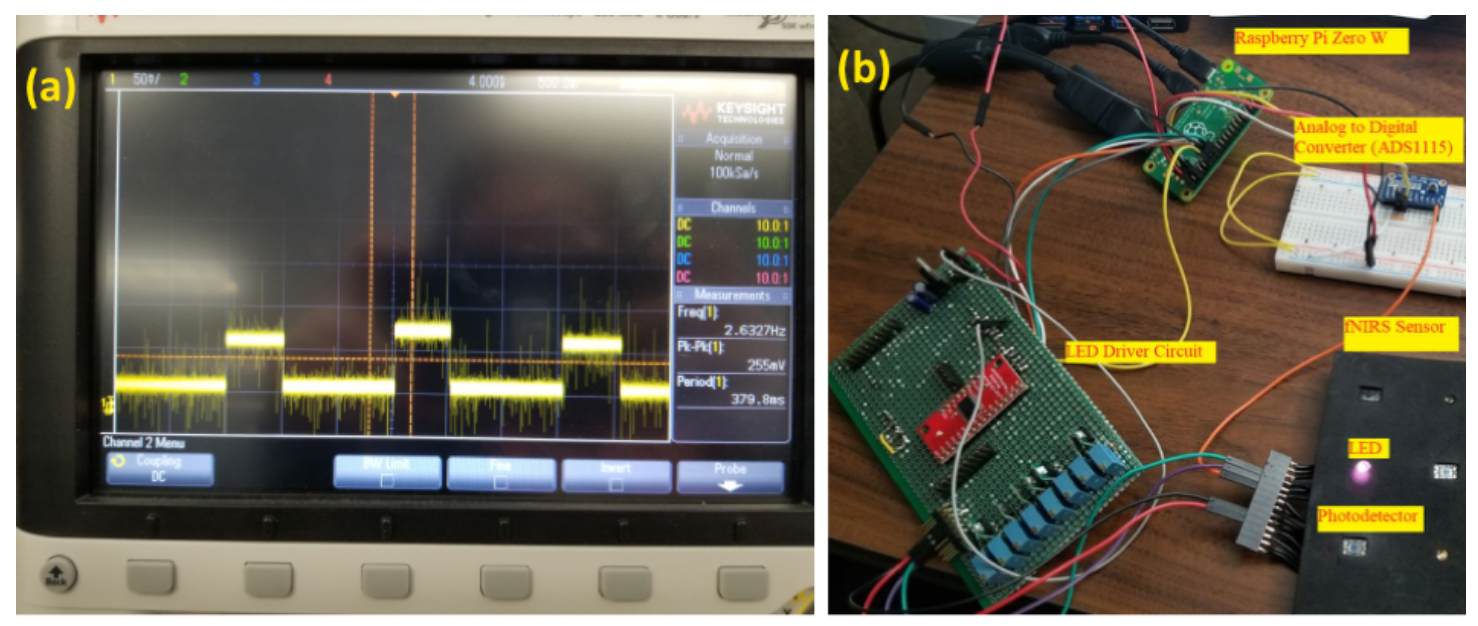

Figure 21. fNIRS hardware testing. (a) photodetector signal on digital oscilloscope, (b) testing of fNIRS hardware components.

types and select them by using push buttons. When the pushbutton is pressed, the GUI sends a command to connect with embedded board wirelessly and then runs the program. Figure 22 shows the screenshot of the GUI. The fNIRS system is also readily accessible via the command prompt. To facilitate easier use, a GUI has also been added to the fNIRS system. Written in Python 2 (PY2) using the pyqt4 and pexpect libraries, this GUI should make adoption of the system easier across multiple platforms and user communities. The program flow used by the application is illustrated in Figure 20. Unless there is a network related issue, the secure shell connection ( $\mathrm{SSH}$ ) is maintained until the user actively disconnects. During this time different buttons on the GUI are associated with issuing specific commands which launch shell scripts on the embedded board. While the GUI is non-blocking when issuing SSH commands, the remote fNIRS device is blocking. This use pattern was selected as only one file or program should actively be accessing the hardware at any time to prevent clobbering. A lock file is written, and the associated PID is recorded. When one of the scripts of the same cohort is executed, a check is made to see if another is currently accessing the hardware and if so, the user is presented with the option to terminate one of them. The GUI 
also provides the ability to remotely retrieve, view, and save a rendered spectra plot since the fNIRS device renders the data locally on completion of a montage.
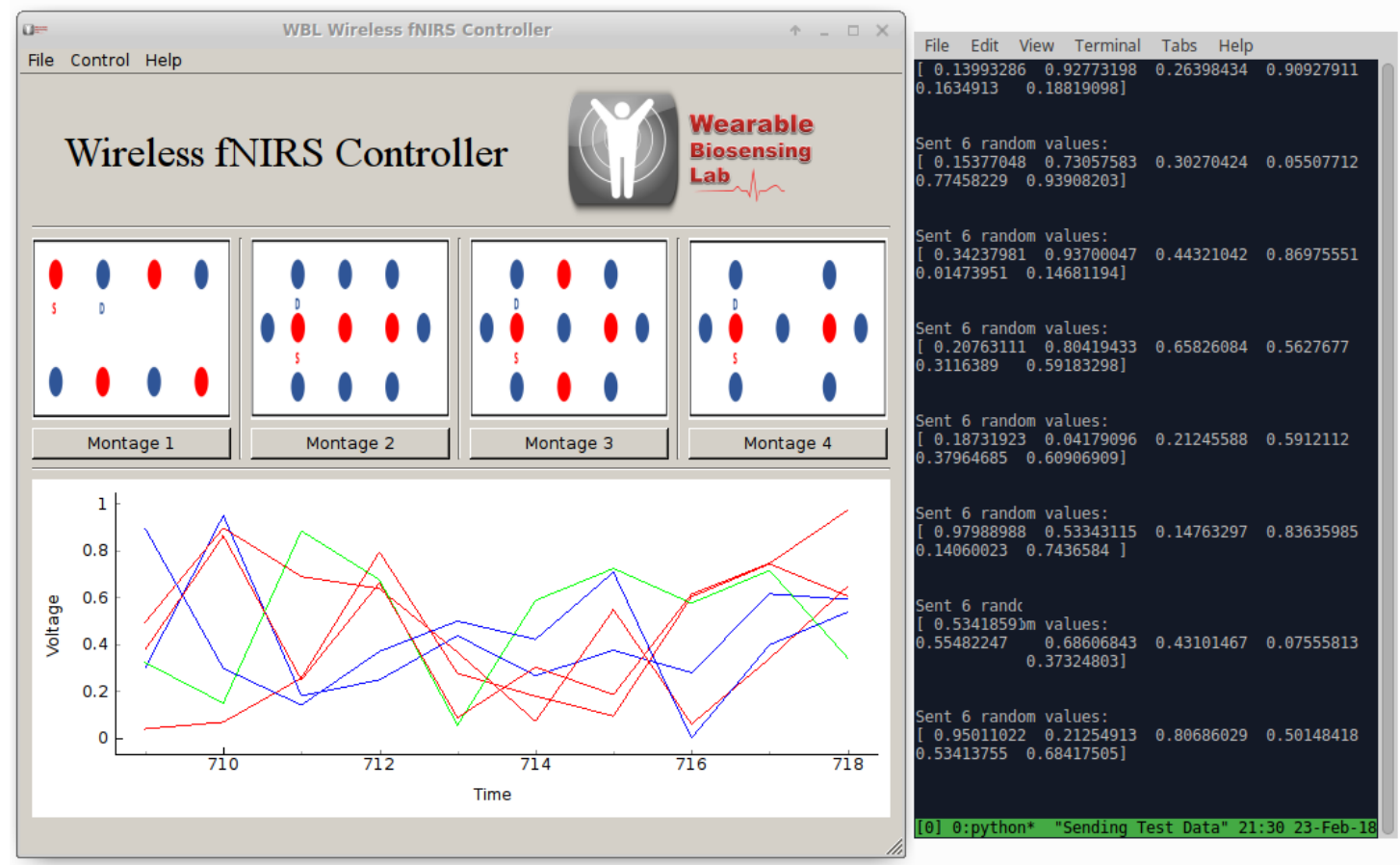

Figure 22. Data communication between fNIRS hardware and graphical user interface (GUI) testing. GUI displays montages and plots channel data in realtime.

The algorithm of application which is shown in Figure 20 is explained below:

- The user launches the GUI from the computer.

- To connect to the embedded board, user sets the username and password.

- After setting the credentials, the user connects to the Raspberry Pi by using the "Connect" button.

- After the connection between Raspberry Pi and computer is established, the user runs "Signal Quality Check" service. This service allows user to check their optodes placement and to verify the proper optode-scalp coupling. 
- After checking the signal quality, the user selects one of the pre-loaded montages and runs the service.

- The pre-loaded code for selected montage runs inside Raspberry Pi and sends the data to the computer. If a data has already been recorded, it can be sent as a single rendered image and displayed by the GUI or in incoming chunks of raw data and rendered directly in the GUI.

- The computer displays and visualizes the data.

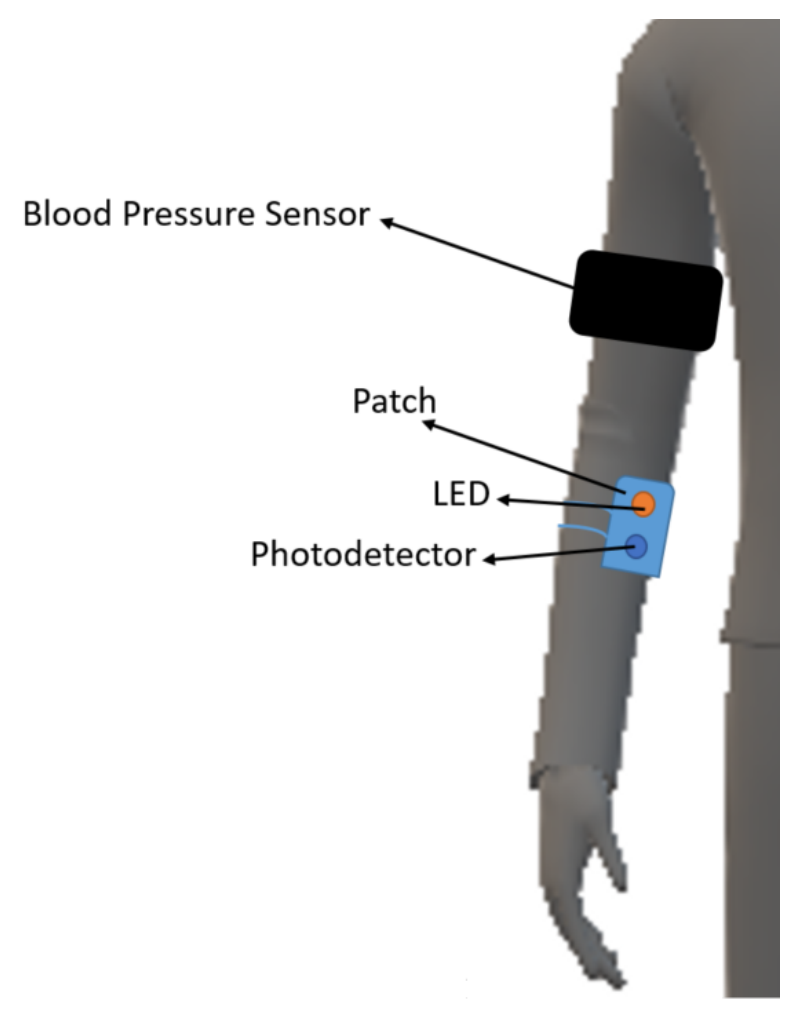

Figure 23. Experimental setup for the arterial occlusion test.

\subsection{Results and Discussion}

\subsection{1 fNIRS Hardware Evaluation}

The proposed system was fabricated on a general purpose PCB and breadboard for the initial validation and functional testing as shown in the Figure 21 (b). The LED triggering signals from the Raspberry Pi to the LED driver circuit 
were tested and successfully verified. The current and LED optical power were also measured. The photodiodes' analog outputs were sent to the ADC. The ADC digitized the data and sent to the Raspberry Pi via $\mathrm{I} 2 \mathrm{C}$ communication. Figure 21 (a) shows the signal coming from photodetectors seen on the oscilloscope.

\subsubsection{Wireless Communication Testing}

To check the wireless communication, both transmission forms were tested by sending data on the same network. The experimental results proved that we were able to establish a reliable, wireless, real-time data communication between the fNIRS system and GUI. According to the algorithm explained in the section 3.4.3, first, we set the IP address of embedded board, username and password. Then we connected the GUI to the system. Then, we checked the signal coming from photodetectors. Figure 22 shows the incoming test data. The data collected from photodetectors also displayed on fNIRS system screen and sent to the GUI on the end user's computer via TCP/IP connection. The GUI plotted the data.

\subsubsection{System Performance Evaluation}

We have performed a simple experiment, arterial occlusion test to evaluate the working of the system. This experiment can evaluate the working of an optical imaging system in measuring hemoglobin concentration change [35]. For this experiment, the fNIRS sensor was placed on the arm, right below the elbow and a blood pressure monitor was placed above the elbow. A 5 minutes trial was performed to capture the changes in the blood flow. First, a 1 minutes rest block was applied. Then, the artery covered by the muscle was occluded by using handcuff of blood pressure monitor for a duration of 2 minutes. After this block, the arm was released for another 2 minutes. Figure 23 demonstrates the experiment setup. The GUI collected data and displayed, and also stored in a .csv file in the user's 


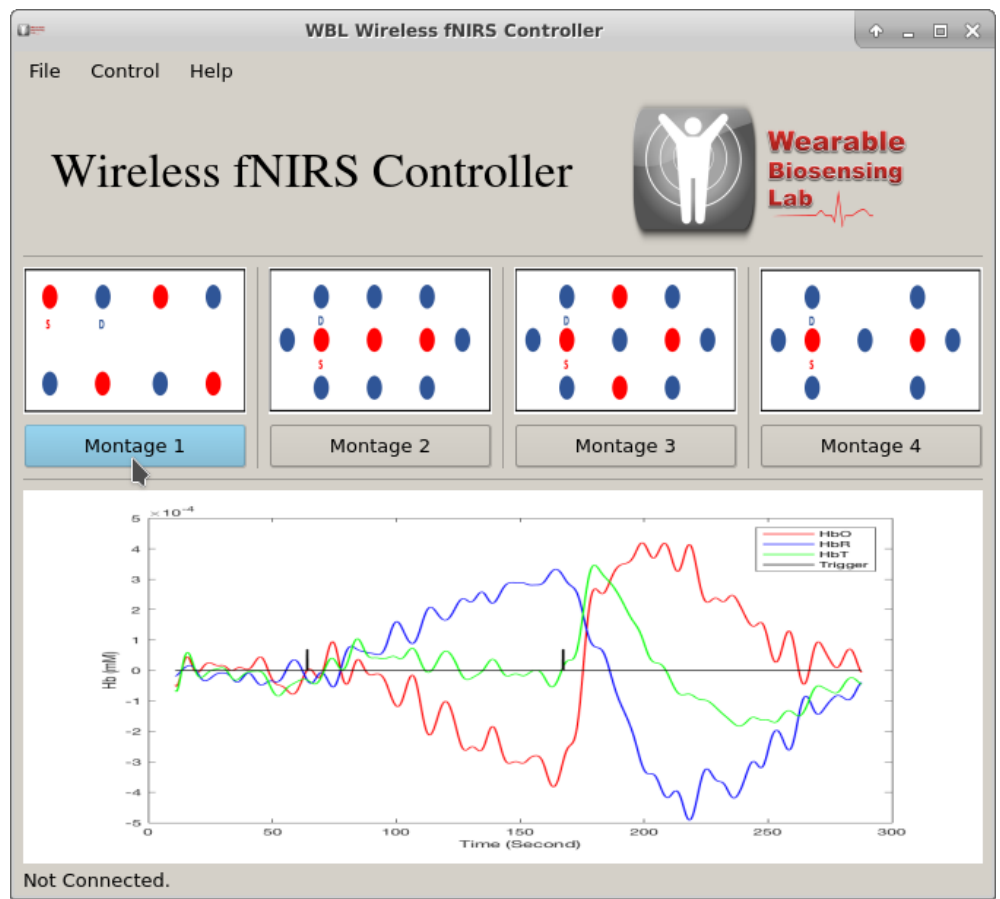

Figure 24. The fNIRS GUI displaying montages and plotting the hemodynamic response.

computer. In user's computer, a custom MatLab script processes the data in background and GUI plots the result. The system detected the changes oxygenated $\left(\mathrm{HbO}_{2}\right)$ and deoxygenated $(\mathrm{Hb})$ hemoglobin concentration during rest time and occlusion successfully. The results are shown in Figure 24.

\subsection{Conclusion and Future Work}

The paper presents a novel design of an fNIRS system with a configurable, service-oriented architecture that communicates with a remote GUI from where experimenter can select the montage wirelessly, test the optode-scalp coupling and SNR, and collect data in real-time. The hardware components of the system were fabricated on general purpose PCB and breadboard for initial validation of the system. A complete service oriented software architecture and GUI were designed in Python programming platform. The communication between the fNIRS device and GUI in a computer was established via wireless TCP/IP communication 
protocol and the data was successfully transmitted in real-time.

The GUI plots the received data in real-time. Since pyqt4 library is stable and well-tested, we used Python 2 programming language to import pyqt4 library. For the future investigation, we shall integrate an external programmable amplifier to increase signal level of the detectors. Also, we shall package all the components in a single PCB and enclose the system in a well-protected cover. In this preliminary design, we used four LEDs and four detectors to keep the system simple; however, in future experiments, the number of sources and detectors can be easily increased to cover the full head. The simple arterial occlusion experiment detected the changes in oxygenation level in the muscle tissue of forearm. In this paper, our main focus was to develop hardware and software architecture for a fNIRS system that can be consider as a smart wearable BSN device. This fNIRS device also successfully collected fNIRS data from brain. However, advanced data processing strategies and topographic plot of brain are still under development. We shall perform brain imaging using a dedicated experimental protocol to visualize the hemodynamic response of the brain in our future work.

\section{Acknowledgment}

This material is based upon work supported by the National Science Foundation under Grant No. 1539068. Gozde Cay is supported by Turkish Ministry of National Education with the full scholarship during her doctoral research.

\section{List of References}

[1] J. B. Balardin, G. A. Zimeo Morais, R. A. Furucho, L. Trambaiolli, P. Vanzella, C. Biazoli, and J. R. Sato, "Imaging brain function with functional near-infrared spectroscopy in unconstrained environments," Frontiers in Human Neuroscience, vol. 11, p. 258, 2017.

[2] M. A. Yücel, J. J. Selb, T. J. Huppert, M. A. Franceschini, and

D. A. Boas, "Functional near infrared spectroscopy: Enabling routine functional brain imaging," Current Opinion in Biomedical Engineering, 
vol. 4, no. Supplement C, pp. 78 - 86, 2017. [Online]. Available: http://www.sciencedirect.com/science/article/pii/S2468451117300697

[3] R. Nishiyori, S. Bisconti, and B. Ulrich, "Motor cortex activity during functional motor skills: An fnirs study," Brain Topography, vol. 29, no. 1, pp. 42-55, Jan 2016.

[4] M. Sood, P. Besson, M. Muthalib, U. Jindal, S. Perrey, A. Dutta, and M. Hayashibe, "Nirs-eeg joint imaging during transcranial direct current stimulation: Online parameter estimation with an autoregressive model," Journal of Neuroscience Methods, vol. 274, pp. 71 - 80, 2016.

[5] F. Wallois, M. Mahmoudzadeh, A. Patil, and R. Grebe, "Usefulness of simultaneous eeg-nirs recording in language studies," Brain and Language, vol. 121, no. 2, pp. 110 - 123, 2012, functional NearInfra Red Spectroscopy (fNIRS): A Promising Functional Imaging Technique for the Study of Brain and Language. [Online]. Available: http://www.sciencedirect.com/science/article/pii/S0093934X11000599

[6] F. F. Jöbsis, "Noninvasive, infrared monitoring of cerebral and myocardial oxygen sufficiency and circulatory parameters," Science, vol. 198, no. 4323, pp. 1264-1267, 1977. [Online]. Available: http://www.jstor.org/stable/1745848

[7] S. Ahn and S. C. Jun, "Multi-modal integration of eeg-fnirs for brain-computer interfaces - current limitations and future directions," Frontiers in Human Neuroscience, vol. 11, p. 503, 2017.

[8] M. J. Saikia, W. Besio, and K. Mankodiya, "Wearlight: Towards a wearable, configurable functional nir spectroscopy system for noninvasive neuroimaging," IEEE Transactions on Biomedical Circuits and Systems, pp. 1-1, 2018.

[9] M. H. J. C. E. Anna Blasi, Sarah Lloyd-Fox, "Test-retest reliability of functional near infrared spectroscopy in infants," Neurophotonics, vol. 1, pp. 1 $1-12,2014$.

[10] M. Ferrari and V. Quaresima, "A brief review on the history of human functional near-infrared spectroscopy (fnirs) development and fields of application," NeuroImage, vol. 63, no. 2, pp. 921 - 935, 2012. [Online]. Available: http://www.sciencedirect.com/science/article/pii/ S1053811912003308

[11] M. J. Saikia and R. Kanhirodan, "Region-of-interest diffuse optical tomography system," Review of Scientific Instruments, vol. 87, no. 1, p. 013701, 2016. [Online]. Available: https://doi.org/10.1063/1.4939054

[12] N. M. Gregg, B. R. White, B. W. Zeff, A. J. Berger, and J. P. Culver, "Brain specificity of diffuse optical imaging: Improvements from superficial signal regression and tomography," Frontiers in Neuroenergetics, vol. 2, p. 14, 2010. 
[13] M. J. Saikia, R. Manjappa, K. Mankodiya, and R. Kanhirodan, "Depth sensitivity improvement of region-of-interest diffuse optical tomography from superficial signal regression," in Imaging and Applied Optics 2018 (3D, AO, AIO, COSI, DH, IS, LACSEA, LSEC, MATH, pcAOP). Optical Society of America, 2018, p. CM3E.5. [Online]. Available: http://www.osapublishing.org/abstract.cfm?URI=COSI-2018-CM3E.5

[14] A. T. Eggebrecht, S. L. Ferradal, A. Robichaux-Viehoever, M. S. Hassanpour, H. Dehghani, A. Z. Snyder, T. Hershey, and J. P. Culver, "Mapping distributed brain function and networks with diffuse optical tomography," Nature Photonics, vol. 8, pp. 448 EP -, 05 2014. [Online]. Available: http://dx.doi.org/10.1038/nphoton.2014.107

[15] A. Gibson, T. Austin, N. Everdell, M. Schweiger, S. Arridge, J. Meek, J. Wyatt, D. Delpy, and J. Hebden, "Three-dimensional whole-head optical tomography of passive motor evoked responses in the neonate," NeuroImage, vol. 30, no. 2 , pp. $521-528,2006$.

[16] "Wearable devices that have an eeg sensor," Vandrico Inc.

[17] S. Brigadoi, L. Ceccherini, S. Cutini, F. Scarpa, P. Scatturin, J. Selb, L. Gagnon, D. A. Boas, and R. J. Cooper, "Motion artifacts in functional near-infrared spectroscopy: A comparison of motion correction techniques applied to real cognitive data," NeuroImage, vol. 85, pp. 181 - 191, 2014, celebrating 20 Years of Functional Near Infrared Spectroscopy (fNIRS). [Online]. Available: http://www.sciencedirect.com/science/article/ pii/S1053811913004291

[18] "Optical absorption of hemoglobin." [Online]. Available: http://omlc.ogi. edu/spectra/

[19] S. P. Y. B. R. R. A. Laura A. Sordillo, Yang Pu, "Deep optical imaging of tissue using the second and third near-infrared spectral windows," Journal of Biomedical Optics, vol. 19, pp. 19 - 19-6, 2014.

[20] M. J. Saikia, R. Manjappa, and R. Kanhirodan, "A cost-effective led and photodetector based fast direct $3 \mathrm{~d}$ diffuse optical imaging system," in Diffuse Optical Spectroscopy and Imaging VI. Optical Society of America, 2017, p. 104120Y. [Online]. Available: http: //www.osapublishing.org/abstract.cfm?URI=ECBO-2017-104120Y

[21] D. T. Delpy, M. Cope, P. van der Zee, S. Arridge, S. Wray, and J. Wyatt, "Estimation of optical pathlength through tissue from direct time of flight measurement," Physics in Medicine 83 Biology, vol. 33, no. 12, p. 1433, 1988. [Online]. Available: http://stacks.iop.org/0031-9155/33/i=12/a=008 
[22] M. J. Saikia and R. Kanhirodan, "Development of dot system for roi scanning," in 12th International Conference on Fiber Optics and Photonics. Optical Society of America, 2014, p. T3A.4. [Online]. Available: http://www.osapublishing.org/abstract.cfm?URI=Photonics-2014-T3A.4

[23] H. Dehghani, B. R. White, B. W. Zeff, A. Tizzard, and J. P. Culver, "Depth sensitivity and image reconstruction analysis of dense imaging arrays for mapping brain function with diffuse optical tomography," Appl. Opt., vol. 48, no. 10, pp. D137-D143, Apr 2009.

[24] M. J. Saikia, R. Kanhirodan, and R. Mohan Vasu, "High-speed gpu-based fully three-dimensional diffuse optical tomographic system," International Journal of Biomedical Imaging, vol. 2014, no. 376456, p. 13, 2014. [Online]. Available: http://dx.doi.org/10.1155/2014/376456

[25] M. J. Saikia, R. Kanhirodan, and R. M. Vasu, "High-speed gpu-based fully three-dimensional diffuse optical tomographic system," Journal of Biomedical Imaging, vol. 2014, pp. 3:3-3:3, Jan. 2014. [Online]. Available: http://dx.doi.org/10.1155/2014/376456

[26] D. K. Nguyen, J. Tremblay, P. Pouliot, P. Vannasing, O. Florea, L. Carmant, F. Lepore, M. Sawan, F. Lesage, and M. Lassonde, "Non-invasive continuous eeg-fnirs recording of temporal lobe seizures," Epilepsy Research, vol. 99, no. 1, pp. 112 - 126, 2012. [Online]. Available: http://www.sciencedirect.com/science/article/pii/S0920121111003627

[27] A. Bozkurt, A. Rosen, H. Rosen, and B. Onaral, "A portable near infrared spectroscopy system for bedside monitoring of newborn brain," BioMedical Engineering OnLine, vol. 4, no. 1, p. 29, Apr 2005. [Online]. Available: https://doi.org/10.1186/1475-925X-4-29

[28] G. Yurtsever, F. Kepics, and A. Bozkurt, "Wireless continuous wave near infrared imager," in 2003 IEEE 29th Annual Proceedings of Bioengineering Conference, March 2003, pp. 53-54.

[29] H. Atsumori, M. Kiguchi, A. Obata, H. Sato, T. Katura, K. Utsugi, T. Funane, and A. Maki, "Development of a multi-channel, portable optical topography system," in 2007 29th Annual International Conference of the IEEE Engineering in Medicine and Biology Society, 2007, pp. 3362-3364.

[30] D. Agrò, R. Canicattì, M. Pinto, G. Morsellino, A. Tomasino, G. Adamo, L. Curcio, A. Parisi, S. Stivala, N. Galioto, et al., "Design and implementation of a portable fnirs embedded system," in Applications in Electronics Pervading Industry, Environment and Society. Springer, 2016, pp. 43-50.

[31] M. Abtahi, G. Cay, M. J. Saikia, and K. Mankodiya, "Designing and testing a wearable, wireless fnirs patch," in 2016 38th Annual International Conference 
of the IEEE Engineering in Medicine and Biology Society (EMBC), Aug 2016, pp. 6298-6301.

[32] J. M. L. E. K. C. G. Alexis Machado, Odile Marcotte, "Optimal optode montage on electroencephalography/functional near-infrared spectroscopy caps dedicated to study epileptic discharges," Journal of Biomedical Optics, vol. 19, pp. $19-19-17,2014$.

[33] D. Sprott and L. Wilkes, "Understanding service-oriented architecture."

[34] "Service-oriented architecture standards," opengroup.org/standards/soa.

[35] Y. Shang, Y. Zhao, R. Cheng, L. Dong, D. Irwin, and G. Yu, "Portable optical tissue flow oximeter based on diffuse correlation spectroscopy," Opt. Lett., vol. 34, no. 22, pp. 3556-3558, Nov 2009. [Online]. Available: http://ol.osa.org/abstract.cfm?URI=ol-34-22-3556 


\title{
MANUSCRIPT 4
}

A wireless fNIRS Patch with short-channel regression to improve detection of hemodynamic response of brain

by

\author{
Manob Jyoti Saikia, and Kunal Mankodiya
}

published in

IEEE International Conference on Electronics, Communication, Computer Technologies and Optimization Techniques (ICEECCOT-2018), Mysuru, India, Dec. 14-15, 2018. 


\subsection{Abstract}

The functional near-infrared spectroscopy (fNIRS) utilizes near-infrared (NIR) light sources and light detectors to non-invasively image hemodynamic responses of the brain. The unique advantages of fNIRS over the other existing brain imaging technologies led to widespread adoption of fNIRS in various brain research studies including neurology, neuroscience, clinical psychology, and psychiatry. The fNIRS systems are portable, inexpensive and provide higher temporal resolution for scanning a brain. In this paper, we present a wireless wearable fNIRS patch that has the capability of short channel regression to improve the detection of hemodynamic responses of the brain. The patch has two targeted fNIRS channels and a short-channel. The short-channel measures the background hemodynamic responses explicitly from the extracerebral region. Then it performs a regression process to eliminate background interferences from the targeted fNIRS channels to reduce the influence of the interferences. We have interfaced the patch with our laboratory-developed portable fNIRS controller. The patch and the controller are wearable. The controller is wirelessly connected to a host computer to receive commands from it and to wirelessly transmit measurement data to the host computer for the data processing and visualization. The graphical user interface (GUI) in the host computer helps the user to record and visualize fNIRS data. The experimental results of imaging prefrontal cortex of the brain using the fNIRS patch show that the patch has the potential to reduce unrelated hemodynamic activity from the targeted fNIRS channels.

\subsection{Introduction}

Functional near-infrared spectroscopy (fNIRS) is an emerging brain imaging technology that non-invasively maps hemodynamic responses of the brain by measuring changes in oxygenated $\left(\mathrm{HbO}_{2}\right)$ and deoxygenated $(\mathrm{Hb})$ hemoglobin con- 


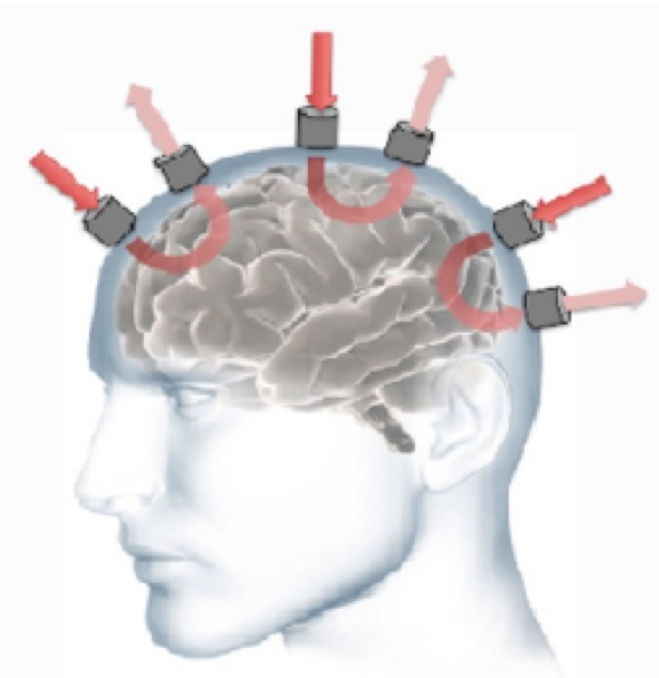

Figure 25. NIR light propagation from sources to detectors carrying information of the hemodynamic activities of the brain.

centration on the cortical surface of the brain $[1,2,3,4,5]$. The fNIRS systems are relatively inexpensive and provide higher temporal resolution compared to its counterpart, functional magnetic resonance imaging (fMRI) [6, 3]. Hence, fNIRS has been widely adopted for clinical settings such as neurology, neuroscience, clinical psychology, and psychiatry[7,3]. Recent advancement in fNIRS hardware for portable brain imaging have prompted a dramatic increase in adoption of fNIRS for brain imaging in the unconstrained environments such as when the participants are playing a sport, playing a musical instrument or interacting in a social situation $[8,9,10]$. Wearable wireless health monitoring devices have a promising future in monitoring the physiological parameter for medical diagnosis. Recently, research and development on wearable electroencephalogram (EEG) monitor have been pursued by research and commercial organizations [11]. Also, portable wearable fNIRS systems are proposed by various research groups $[12,13,14,15,16,17,18,19]$. In addition, multi-model brain imaging combining fNIRS with EEG are suggested to take the complementary benefits of two imaging modalities $[20,21,22]$. However, 
fNIRS as a wearable device requires sophisticated hardware, optodes, and a controlling and data processing algorithm. It demands a power-efficient wireless data transmission to provide flexibility of performing imaging of a moving brain.

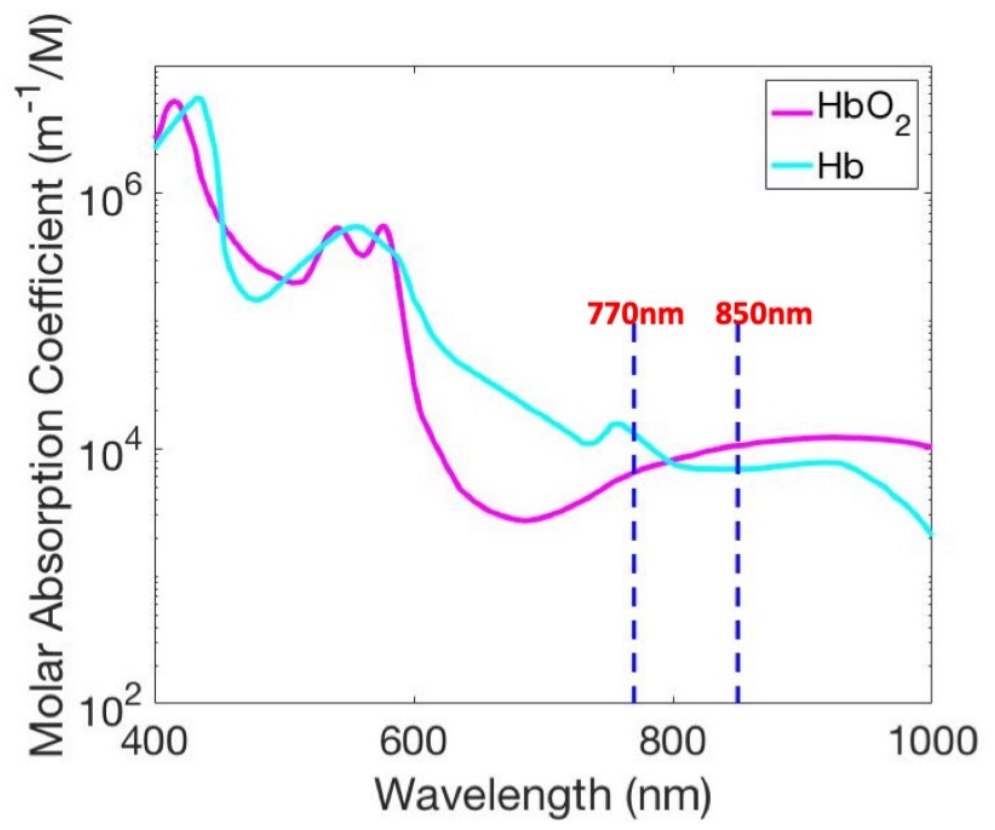

Figure 26. Plot of absorption spectra of $\mathrm{HbO}_{2}$ and $\mathrm{Hb}$ in the NIR window. Experimental data were taken from OMLC [23].

The fNIRS brain imaging systems use NIR light sources in the wavelength range from $600 \mathrm{~nm}$ to $900 \mathrm{~nm}$ and light detectors to place on the scalp in a predefined arrangement. The emitted light from an NIR source travels through the tissue, and the detector detects the back reflected light from the cortical surface as shown in Fig. 25. The penetration depth of the NIR light and fNIRS signal strength largely depend on source-detector separation [24, 25]. The hemodynamic response of the brain also varies with the experimental paradigm and participant. In general, the wearable fNIRS patch uses a single source-detector pair in order to calculate the cerebral hemodynamic response of each fNIRS channels $[12,13,14$, 15, 16, 17, 18, 26]. Each single source-detector pairs create an fNIRS channel. In such cases, isolation of the hemodynamic responses related to the stimulus from 
uncorrelated hemodynamic trends such as respiration and Mayer waves is difficult and it requires extensive post processing of the fNIRS signal.

In this paper, we present a simple wearable fNIRS patch that has the capability of short channel regression to improve the detection of hemodynamic responses of the brain. The patch has two different source-detector separations. It uses a reduced source-detector separation channel (short-channel) to measure the background hemodynamic responses explicitly from the extracerebral region. Then it subtracts off the background interferences from the targeted fNIRS channels to reduce the influence of the interferences. We have interfaced the patch with our laboratory-developed portable fNIRS controller. The patch and the controller are wearable. The controller is wirelessly connected to a host computer to receive commands from it and to wirelessly transmit measurement data to the host computer for the data processing and visualization. The graphical user interface (GUI) in the host computer helps the user to record and visualize fNIRS data. We have

performed experimental studies to image the prefrontal cortex of the brain using the fNIRS patch. The data collected from the patch is processed for the performance analysis. The experimental result shows that the patch has the potential to reduce unrelated hemodynamic activity from the targeted fNIRS channels.

\subsection{Background of fNIRS Imaging}

In fNIRS brain imaging, NIR light source is employed to shine on the scalp and light that propagates through the tissue is mainly absorbed by the $\mathrm{HbO}_{2}$ and $H b$ blood on the cortical area of the brain. The back-reflected light intensity from the cortical surface of the brain is measured using detectors as shown in Fig. 25. The light absorption by the $\mathrm{HbO}_{2}$ and $\mathrm{Hb}$ blood in the brain is wavelength dependent $[23,27]$. Hence, probing with two distinct wavelengths (one wavelength at a time) in the NIR window can resolve $\mathrm{HbO}_{2}$ and $\mathrm{Hb}$ concentration changes on 

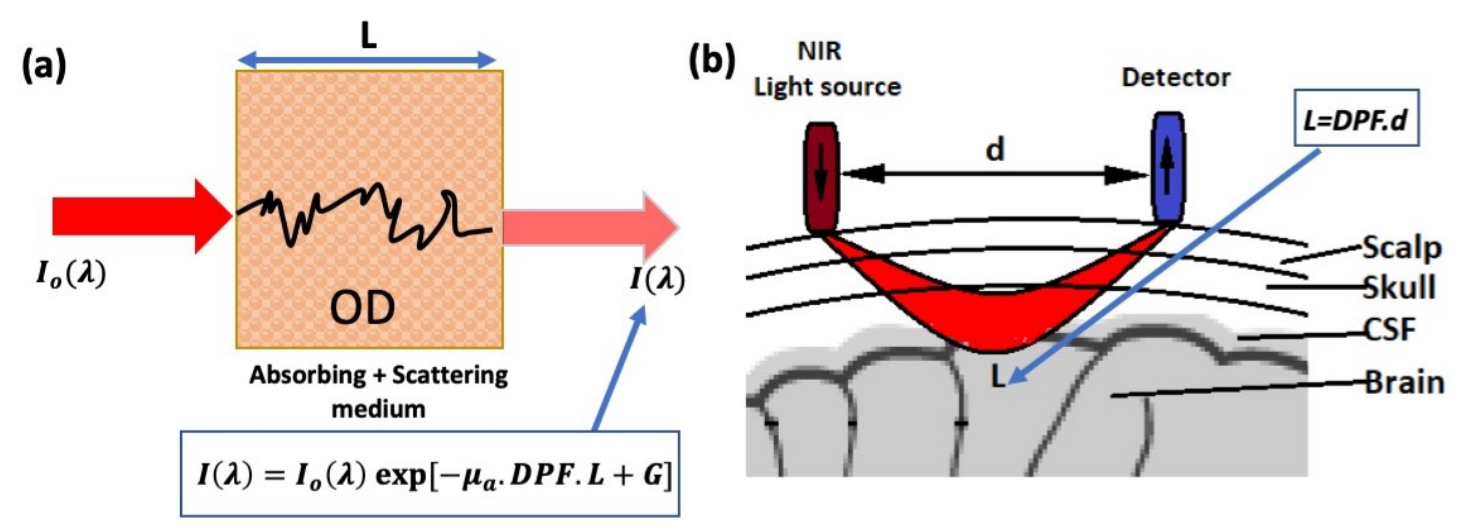

Figure 27. a) Principle of Modified Beer-Lambert Law, and (b) sketch of NIR light propagation from a source to a detector through different tissue layers of a brain. Source and detector separating distance $(d)$ is related to optical path length $(L)$ by $L=D P F . d$.

the cortical surface on the brain over time. We have plotted the absorption spectra of $\mathrm{HbO}_{2}$ and $\mathrm{Hb}$ in Fig. 26. In general, an fNIRS system comprises: (1) fNIRS sensors (optodes) made of light source and photodetector, (2) a control unit which controls the system by switching the light sources and collecting the electrical signal from photodetectors with an controlling algorithm and (3) a computer that collects the raw data, makes necessary computations on it and displays the results.

The hemoglobin concentration changes are calculated using the Modified BeerLambert Law (MBLL) [28, 16]. The principle of MBLL is shown in Fig. 27 (a) where output light $I(\lambda)$ is related to the input $I_{o}(\lambda)$ as,

$$
I=I_{o} \exp \left[-\mu_{a} . D P F \cdot L+G\right]
$$

where $\mathrm{G}$ is loss due to geometry and $\mu_{a}$ is absorption coefficient of the medium.

The distance between the source and detector is important in fNIRS. The optical path length $(L)$ is more than the actual distance between the source and detector $(d)$ as shown in Fig. 27 (b). $L$ is related to $d$ by a unitless quantity, differential pathlength factor (DPF) $[L=D P F . d]$. While the distance (d) increases, 

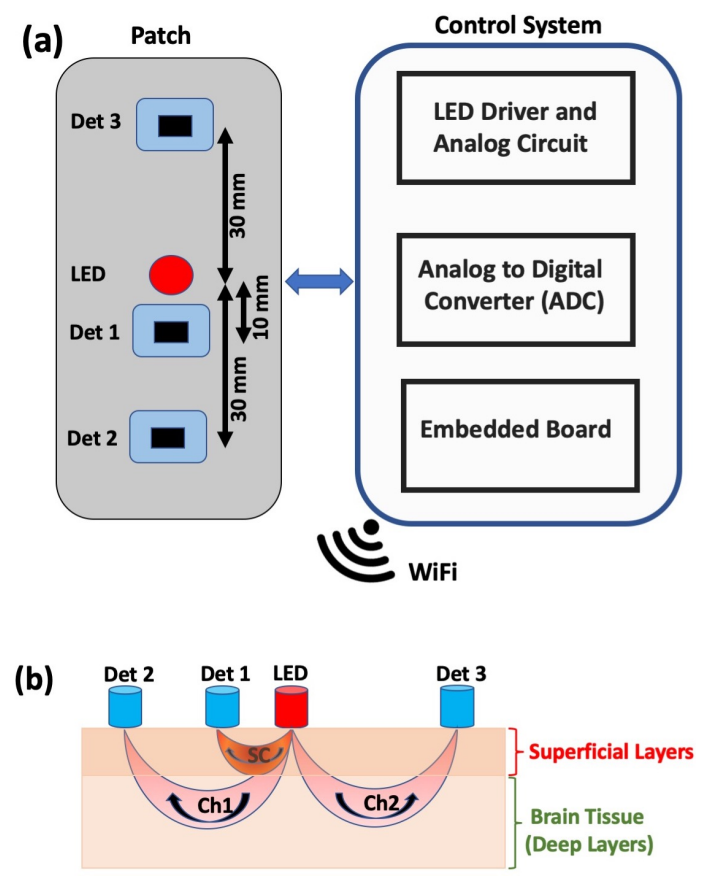

Figure 28. (a) A conceptual diagram of fNIRS patch connected to a wireless fNIRS controller. (b) The patch has one dual-wavelength LED and three detectors forming two target fNIRS channels (Ch1 and Ch2) and a short-channel (SC).

fNIRS signal strength decreases. A $d$ of 30 to $40 \mathrm{~mm}$ provides a detectable signal $[24,29,30]$.

\subsection{Wireless Wearable fNIRS Patch with a Short-channel}

We have proposed a wearable fNIRS patch having a dedicated surface-sensitive fNIRS short-channel to regress out background hemodynamic responses and to isolate activation-specific responses. The patch is interfaced with our fNIRS controlling hardware that comprises of three functional blocks: (1) LED driver and analog circuit, (2) Analog to digital converter, and (3) Embedded board. The patch was interfaced with the fNIRS controller to collect data from participants and to send data wirelessly to a host computer. The fNIRS hardware is controlled by a GUI software from the host computer. 


\subsection{1 fNIRS Optode Patch Design}

The fNIRS source and detector, also known as optodes, are placed on the scalp with a predefined arrangement [31]. Our optode patch has a dual wavelength (770 $\mathrm{nm}$ and $850 \mathrm{~nm}$ ) LED as source optode and three silicon-photodiode detectors as detector optodes. The location of each optode and the distance between source and detector are very important for collecting fNIRS data. The human head has different tissue layers and optical path length and penetration depth vary with source-detector separating distances [29]. The location of the source optode and the detector optodes are shown in Fig. 28 (a). The detector optodes Det 2 and Det 3 are $30 \mathrm{~mm}$ apart from the source optode (LED) creating two main targeted fNIRS channels Ch1 and Ch2 respectively as shown in Fig. 28 (b). The detector optode Det 1 located at $10 \mathrm{~mm}$ from the LED forming a short-channel (SC) [Fig. 28 (a)-(b)]. This $10 \mathrm{~mm}$ short-channel senses the extracerebral hemodynamics. We implement a short-distance correction method to reduce the contribution from the extracerebral hemodynamics to the two target-channel measurements. This should ideally leave brain-only responses behind improving the contrast-to-noise ratio of the fNIRS target channels.

\subsection{2 fNIRS Controlling Hardware}

Our fNIRS controlling system is composed of an embedded board, analog to digital converter and analog circuit as shown in the block diagram in Fig. 28 (a).

\section{Embedded Controller}

The embedded board used to build the fNIRS controller is Raspberry Pi Zero W. The embedded controller coordinates all the tasks including receiving commands from a host computer wirelessly, configuring optodes of an experiment, triggering the LEDs and measuring the detected fNIRS signal during the exper- 


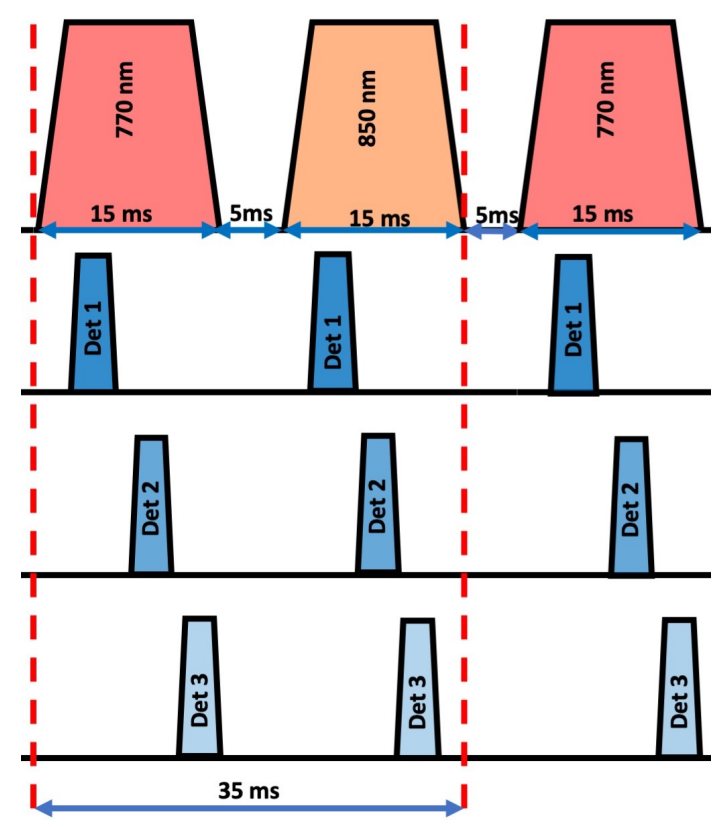

Figure 29. Timing sequences for triggering LED (wavelengths $770 \mathrm{~nm}$ and 850 $\mathrm{nm})$ and three detector readings (Det 1 - Det 3 ).

iment and sending data to the host wirelessly. The embedded board sends the triggering signal to the analog circuits to drive the LED according to a multiplexing algorithm configured by the user. Then it measures the reflected light which is detected by the detectors and digitized by the ADC. In addition, it sends the fNIRS data to the host computer wirelessly to display the fNIRS signal and process. Fig. 29 illustrates dual-wavelength LED triggering and light detection timing sequences we implemented for the patch. Each wavelength of the LED is turned ON for $15 \mathrm{~ms}$ and there is a $5 \mathrm{~ms}$ OFF time between each wavelength. Thus, the total duration of a cycle is $35 \mathrm{~ms}$.

\section{Analog to Digital Converter}

The three detectors embedded in the patch detect back reflected $770 \mathrm{~nm}$ and $850 \mathrm{~nm}$ NIR light (one wavelength at a time) and output readings as analog electrical signal. We have used a 16-bit 4-channel precision Analog to Digital Converter (ADC), ADS1115 to sequentially convert the electrical readings from the detec- 


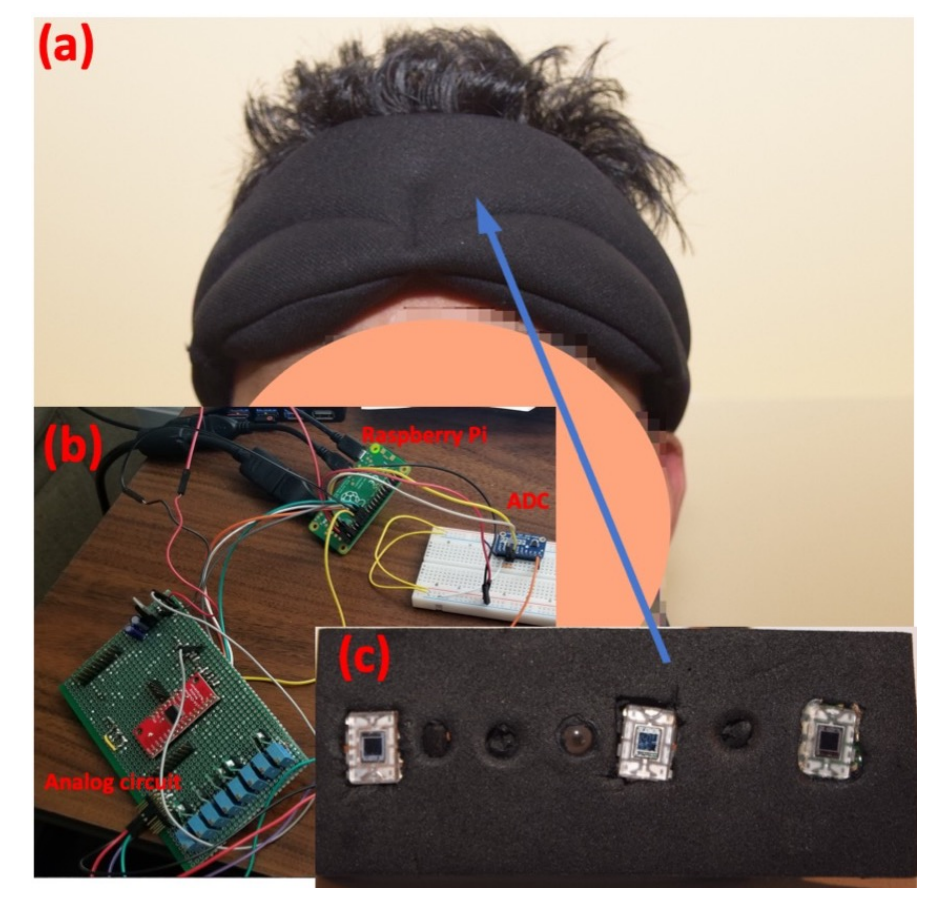

Figure 30. (a) Participant wearing the fNIRS patch, (b) fNIRS hardware testing setup, and (c) fNIRS patch.

tors to digital signal. The ADC is controlled by the embedded controller via $\mathrm{I} 2 \mathrm{C}$ communication protocol. The ADC readings are transferred to the embedded computing module for the data conversion and wireless data transmission to a host computer.

\section{Analog Circuit}

The analog circuit was designed to provide constant and adjustable current to the LED. The analog circuit has the connectors to interface the optode patch. It also has a power management circuit to distribute regulated bias voltages and reference voltages to each of the circuit components, detectors, and ADC. A 9-volt battery is connected to the analog circuit that makes the system safe and wearable. 


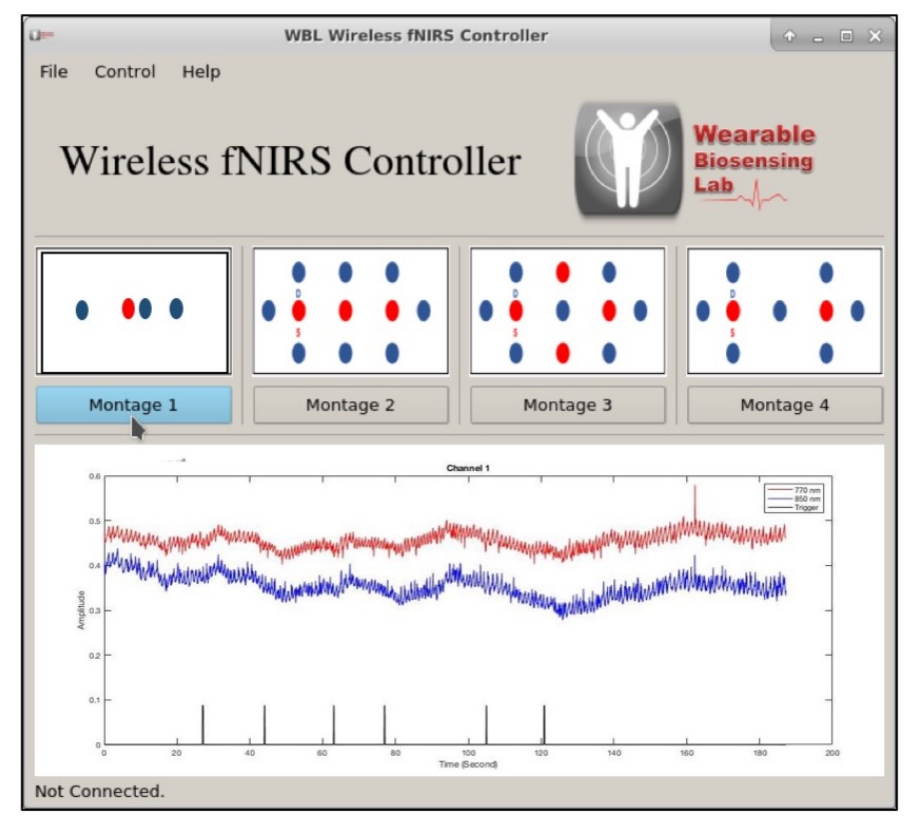

Figure 31. GUI software to control the fNIRS system.

\subsection{Experimental Studies}

We have performed experimental studies by placing the fNIRS patch on the prefrontal cortex of the brain to measure the brain activation due to the cognitive tasks as shown in the Fig. 30 (a).

\subsubsection{Hardware and Software Setup}

Figure 30 (b) presents the fNIRS hardware testing setup containing the Raspberry Pi Zero W, ADS1115 ADC, and an analog circuit. The fNIRS patch has an LED (MTMD7885NN24) and three detectors (OPT 101) as shown in the Fig.30 (c). A sponge headband was used to place the fNIRS patch on the prefrontal lobe of the brain and to make a comfortable optode-scalp interface as shown in the Fig.30 (a). A GUI software as shown in Fig. 31 interfaces the fNIRS system to a computer wirelessly. The GUI utilizes a service-oriented architecture (SOA) to provide various services such as, checking the signal quality and the optode placement, fNIRS channel selection, start and stop an experiment, displaying raw 
data and plotting, intensive post-processing and brain signal visualization. Data was collected from two targeted fNIRS channels (Ch1 and Ch2) and the shortchannel (SC) using the GUI software. The time series raw data was converted to the hemodynamic responses of the brain using MBLL [Fig. 27]. We also used 10 $\mathrm{mm}$ short-channel data to correct the hemodynamic responses of the two targeted fNIRS channels.
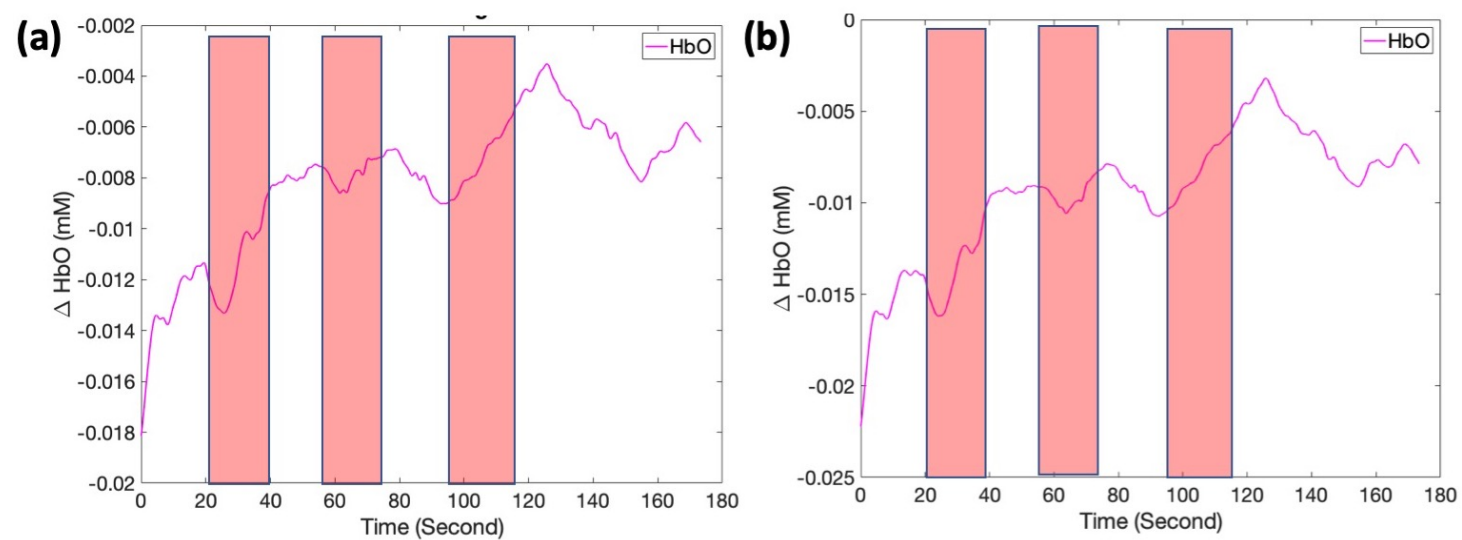

Figure 32. Oxyhemoglobin concentration changes. (a) and (b) are the fNIRS channels Ch1 and Ch2 using detector 2 and detector 3 reading alone respectively. Red blocks are the stimulation epochs.

\subsubsection{Experimental Protocol}

Participants wearing the fNIRS patch on the prefrontal region of the brain were performing mental arithmetic tasks during the course of the experiment in order to increase the cognitive load. The experimental protocol involved participant sitting about $65 \mathrm{~cm}$ away in front of a computer screen. The screen displayed computer generated random arithmetic tasks with the difficulty levels in the increasing order. This slowly increased cognitive load and anxiety of the subject, and it also demanded usage of more working memory. We incorporated three incremental difficulty levels with the time window of about 20 seconds. The solid red blocks in Fig. 32, 33 and 34 represent the time windows (epochs) when the 
computer screen displayed the mental arithmetic tasks of the three difficulty levels.

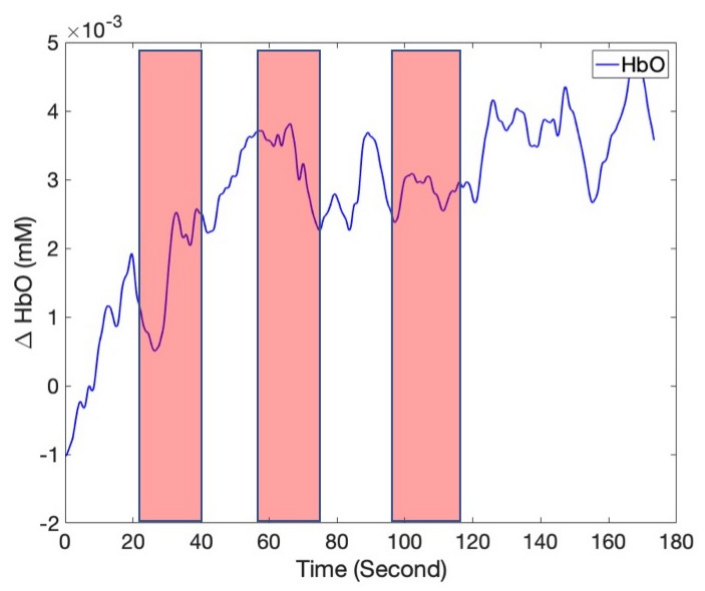

Figure 33. Oxyhemoglobin concentration changes of short-channel using detector 1. Red blocks are the stimulation epochs.

\subsection{Results and Discussion}

We calculated changes in concentrations of oxygenated hemoglobin $\left(\mathrm{HbO}_{2}\right)$ in the prefrontal cortex using our MatLab based code implementing MBLL [Eq. 3]. The raw intensity data was converted to the relative changes in $\Delta \mathrm{HbO}_{2}$ for each fNIRS channels. We found that hemodynamic response to the cortical activity rises in response to the mental arithmetic over time. Every target channel's time series of oxyhemoglobin concentration change was processed using no regressor at all for the comparison. A separate processing with short-channel correction was performed to compare the performance. The regression process involves scaled subtraction of the short-channel by least-squares minimization that produces a corrected time series [30, 32]. Figure 32 (a) and (b) presents the oxyhemoglobin changes $\mathrm{HbO}_{2}$, calculated using the two target channels' detector reading alone. These two targeted channels (Ch1 and Ch2) have the source-detector spacing of $30 \mathrm{~mm}$. The oxyhemoglobin changes of the short-channel (SC) having the sourcedetector spacing of $10 \mathrm{~mm}$ is shown in Fig. 33. The short-channel is sensitive 
to the superficial hemodynamic of the extracerebral tissue layer. Figure 34 (a) and (b) are the corrected responses using superficial signal regression. These corrected responses exhibit a much stronger correlation with the stimulus onsets than the original signal. This result shows that fNIRS patch with a surface-sensitive recording using a short source-detector separation helps to eliminate nonspecific hemodynamic trends from the stimulus-evoked hemodynamic response in the targeted fNIRS channels.
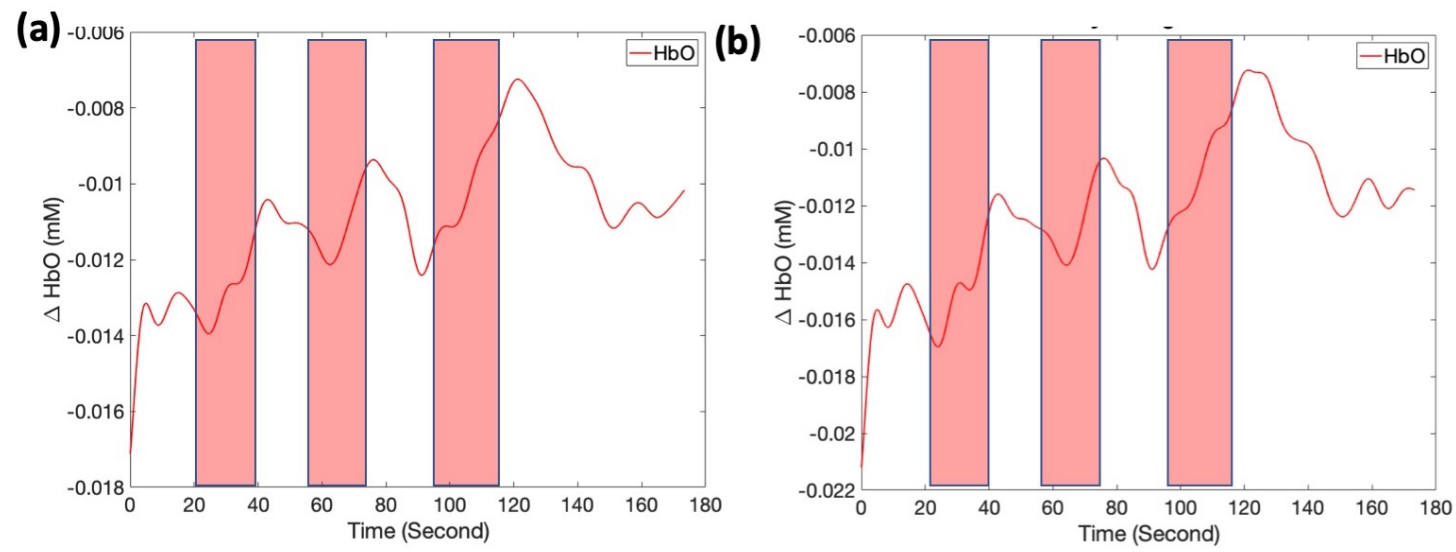

Figure 34. Corrected oxyhemoglobin trends using short-channel data regression. (a) and (b) are the corrected fNIRS channels Ch1 and Ch2. Red blocks are the stimulation epochs.

\subsection{Conclusion and Future Work}

We have designed a wearable fNIRS patch that has a regression channel with a source-detector spacing of $10 \mathrm{~mm}$ (short-channel) and two main target channels with the source-detector spacing of $30 \mathrm{~mm}$ respectively. We have evaluated the effectiveness of superficial signal regression of the wireless fNIRS patch. The experimental result shows that the patch has the potential to reduce unrelated hemodynamic activity from the fNIRS channels. This fNIRS patch is scalable to increase the number of source and detector. 


\section{Acknowledgment}

This material is based upon work supported by the National Science Foundation under Grant No. 1539068. The authors would also like to thank Gozde Cay and Joshua V. Gyllinsky for their assistance.

\section{List of References}

[1] F. F. Jöbsis, "Noninvasive, infrared monitoring of cerebral and myocardial oxygen sufficiency and circulatory parameters," Science, vol. 198, no. 4323, pp. 1264-1267, 1977. [Online]. Available: http://www.jstor.org/stable/1745848

[2] M. Ferrari and V. Quaresima, "A brief review on the history of human functional near-infrared spectroscopy (fnirs) development and fields of application," NeuroImage, vol. 63, no. 2, pp. 921 - 935, 2012. [Online]. Available: http://www.sciencedirect.com/science/article/pii/ S1053811912003308

[3] F. Irani, S. M. Platek, S. Bunce, A. C. Ruocco, and D. Chute, "Functional near infrared spectroscopy (fnirs): An emerging neuroimaging technology with important applications for the study of brain disorders," The Clinical Neuropsychologist, vol. 21, no. 1, pp. 9-37, 2007, pMID: 17366276. [Online]. Available: https://doi.org/10.1080/13854040600910018

[4] M. J. Saikia, R. Kanhirodan, and R. Mohan Vasu, "High-speed gpu-based fully three-dimensional diffuse optical tomographic system," International Journal of Biomedical Imaging, vol. 2014, no. 376456, p. 13, 2014. [Online]. Available: http://dx.doi.org/10.1155/2014/376456

[5] U. Chaudhary, N. Birbaumer, and A. Ramos-Murguialday, "Braincomputer interfaces for communication and rehabilitation," Nature Reviews Neurology, vol. 12, pp. 513 EP -, 08 2016. [Online]. Available: http://dx.doi.org/10.1038/nrneurol.2016.113

[6] N. K. Logothetis, "What we can do and what we cannot do with fmri," Nature, vol. 453, no. 7197, pp. 869-878, 2008.

[7] A.-C. Ehlis, S. Schneider, T. Dresler, and A. J. Fallgatter, "Application of functional near-infrared spectroscopy in psychiatry," NeuroImage, vol. 85, pp. 478 - 488, 2014, celebrating 20 Years of Functional Near Infrared Spectroscopy (fNIRS). [Online]. Available: http://www.sciencedirect.com/ science/article/pii/S1053811913003200

[8] J. B. Balardin, G. A. Zimeo Morais, R. A. Furucho, L. Trambaiolli, P. Vanzella, C. Biazoli, and J. R. Sato, "Imaging brain function with func- 
tional near-infrared spectroscopy in unconstrained environments," Frontiers in Human Neuroscience, vol. 11, p. 258, 2017.

[9] M. A. Yücel, J. J. Selb, T. J. Huppert, M. A. Franceschini, and D. A. Boas, "Functional near infrared spectroscopy: Enabling routine functional brain imaging," Current Opinion in Biomedical Engineering, vol. 4, no. Supplement C, pp. 78 - 86, 2017. [Online]. Available: http://www.sciencedirect.com/science/article/pii/S2468451117300697

[10] V. Quaresima and M. Ferrari, "Functional near-infrared spectroscopy (fnirs) for assessing cerebral cortex function during human behavior in natural/social situations: A concise review," Organizational Research Methods, p. 1094428116658959, 2018/01/21 2016. [Online]. Available: https://doi.org/10.1177/1094428116658959

[11] "Wearable devices that have an eeg sensor," Vandrico Inc.

[12] A. Bozkurt, A. Rosen, H. Rosen, and B. Onaral, "A portable near infrared spectroscopy system for bedside monitoring of newborn brain," BioMedical Engineering OnLine, vol. 4, no. 1, p. 29, Apr 2005. [Online]. Available: https://doi.org/10.1186/1475-925X-4-29

[13] D. Chitnis, D. Airantzis, D. Highton, R. Williams, P. Phan, V. Giagka, S. Powell, R. J. Cooper, I. Tachtsidis, M. Smith, C. E. Elwell, J. C. Hebden, and N. Everdell, "Towards a wearable near infrared spectroscopic probe for monitoring concentrations of multiple chromophores in biological tissue in vivo," Review of Scientific Instruments, vol. 87, no. 6, p. 065112, 2016. [Online]. Available: https://doi.org/10.1063/1.4954722

[14] D. Agrò, R. Canicattì, M. Pinto, G. Morsellino, A. Tomasino, G. Adamo, L. Curcio, A. Parisi, S. Stivala, N. Galioto, et al., "Design and implementation of a portable fnirs embedded system," in Applications in Electronics Pervading Industry, Environment and Society. Springer, 2016, pp. 43-50.

[15] M. Izzetoglu, K. Izzetoglu, S. Bunce, H. Ayaz, A. Devaraj, B. Onaral, and K. Pourrezaei, "Functional near-infrared neuroimaging," IEEE Transactions on Neural Systems and Rehabilitation Engineering, vol. 13, no. 2, pp. 153-159, 2005.

[16] M. Firbank, E. Okada, and D. T. Delpy, "A theoretical study of the signal contribution of regions of the adult head to near-infrared spectroscopy studies of visual evoked responses," NeuroImage, vol. 8, no. 1, pp. $69-78$, 1998. [Online]. Available: http://www.sciencedirect.com/science/article/pii/ S1053811998903482 
[17] G. Yurtsever, F. Kepics, and A. Bozkurt, "Wireless continuous wave near infrared imager," in 2003 IEEE 29th Annual Proceedings of Bioengineering Conference, March 2003, pp. 53-54.

[18] H. Atsumori, M. Kiguchi, A. Obata, H. Sato, T. Katura, K. Utsugi, T. Funane, and A. Maki, "Development of a multi-channel, portable optical topography system," in 2007 29th Annual International Conference of the IEEE Engineering in Medicine and Biology Society, 2007, pp. 3362-3364.

[19] M. J. Saikia, W. Besio, and K. Mankodiya, "Wearlight: Towards a wearable, configurable functional nir spectroscopy system for noninvasive neuroimaging," IEEE Transactions on Biomedical Circuits and Systems, pp. 1-1, 2018.

[20] M. Sood, P. Besson, M. Muthalib, U. Jindal, S. Perrey, A. Dutta, and M. Hayashibe, "Nirs-eeg joint imaging during transcranial direct current stimulation: Online parameter estimation with an autoregressive model," Journal of Neuroscience Methods, vol. 274, pp. 71 - 80, 2016.

[21] D. K. Nguyen, J. Tremblay, P. Pouliot, P. Vannasing, O. Florea, L. Carmant, F. Lepore, M. Sawan, F. Lesage, and M. Lassonde, "Non-invasive continuous eeg-fnirs recording of temporal lobe seizures," Epilepsy Research, vol. 99, no. 1, pp. 112 - 126, 2012. [Online]. Available: http://www.sciencedirect.com/science/article/pii/S0920121111003627

[22] S. Ahn and S. C. Jun, "Multi-modal integration of eeg-fnirs for brain-computer interfaces - current limitations and future directions," Frontiers in Human Neuroscience, vol. 11, p. 503, 2017.

[23] "Optical absorption of hemoglobin." [Online]. Available: http://omlc.ogi. edu/spectra/

[24] H. Dehghani, B. R. White, B. W. Zeff, A. Tizzard, and J. P. Culver, "Depth sensitivity and image reconstruction analysis of dense imaging arrays for mapping brain function with diffuse optical tomography," Appl. Opt., vol. 48, no. 10, pp. D137-D143, Apr 2009.

[25] M. J. Saikia and R. Kanhirodan, "Development of dot system for roi scanning," in 12th International Conference on Fiber Optics and Photonics. Optical Society of America, 2014, p. T3A.4. [Online]. Available: http://www.osapublishing.org/abstract.cfm?URI=Photonics-2014-T3A.4

[26] M. Abtahi, G. Cay, M. J. Saikia, and K. Mankodiya, "Designing and testing a wearable, wireless fnirs patch," in 2016 38th Annual International Conference of the IEEE Engineering in Medicine and Biology Society (EMBC), Aug 2016, pp. 6298-6301. 
[27] M. J. Saikia, R. Manjappa, and R. Kanhirodan, "A cost-effective led and photodetector based fast direct 3d diffuse optical imaging system," in Diffuse Optical Spectroscopy and Imaging VI. Optical Society of America, 2017, p. 104120Y. [Online]. Available: http: //www.osapublishing.org/abstract.cfm?URI=ECBO-2017-104120Y

[28] D. T. Delpy, M. Cope, P. van der Zee, S. Arridge, S. Wray, and J. Wyatt, "Estimation of optical pathlength through tissue from direct time of flight measurement," Physics in Medicine $\& 3$ Biology, vol. 33, no. 12, p. 1433, 1988. [Online]. Available: http://stacks.iop.org/0031-9155/33/i=12/a=008

[29] M. J. Saikia and R. Kanhirodan, "Region-of-interest diffuse optical tomography system," Review of Scientific Instruments, vol. 87, no. 1, p. 013701, 2016. [Online]. Available: https://doi.org/10.1063/1.4939054

[30] M. J. Saikia, R. Manjappa, K. Mankodiya, and R. Kanhirodan, "Depth sensitivity improvement of region-of-interest diffuse optical tomography from superficial signal regression," in Imaging and Applied Optics 2018 (3D, AO, AIO, COSI, DH, IS, LACSEA, LSEC, MATH, pсAOP). Optical Society of America, 2018, p. CM3E.5. [Online]. Available: http://www.osapublishing.org/abstract.cfm?URI=COSI-2018-CM3E.5

[31] J. M. L. E. K. C. G. Alexis Machado, Odile Marcotte, "Optimal optode montage on electroencephalography/functional near-infrared spectroscopy caps dedicated to study epileptic discharges," Journal of Biomedical Optics, vol. 19, pp. $19-19-17,2014$.

[32] N. M. Gregg, B. R. White, B. W. Zeff, A. J. Berger, and J. P. Culver, "Brain specificity of diffuse optical imaging: Improvements from superficial signal regression and tomography," Frontiers in Neuroenergetics, vol. 2, p. 14, 2010. 


\section{MANUSCRIPT 5}

Mental workload assessment during n-back working memory task using a portable fNIRS system

by

Manob Jyoti Saikia, Walter G. Besio, and Kunal Mankodiya

Submitted to

IEEE Transactions on Neural Systems and Rehabilitation Engineering 


\subsection{Abstract}

Working memory (WM) is a short-term active memory which is associated with the temporary storage and manipulation of information. WM is important to perform higher-order or regular cognitive tasks. The prefrontal cortex (PFC) brain areas are involved in the processing of working memory. We assessed PFC activation during the n-back working memory task in a group of 13 participants using a laboratory-developed portable functional near-infrared spectroscopy (fNIRS) system (WearLight). We designed an experimental protocol having 16 n-back trials with pseudo-randomized four different task difficulty levels. The hemodynamic response of the brain was computed from the experimental data in the 16 trials and evaluated brains' response due to four different task difficulty levels. We observed incremental mean hemodynamic activation in the group induced by the continuously increasing workload levels in the four-level n-back tasks. Since the portable fNIRS system is wearable and operates wirelessly, it has the potential to measure cognitive load in a naturalistic environment leading to user-friendly BCI development.

\subsection{Introduction}

Human working memory (WM) is a cognitive function that has been extensively researched in cognitive neuroscience [1]. WM is defined as a short-term active memory which is responsible for the temporary storage, continuous updating, processing of remembered information, manipulating stored information, and decision making $[2,3]$. WM is essential to perform higher-order cognitive tasks and everyday activities as well. The information holding and processing capacity of the WM system is limited $[4,1]$ and varies between individuals and tasks. Hence, it can easily reach the limit when cognitive function performs complex tasks involving rapid storing and processing of excessive information in a stressful environment. 


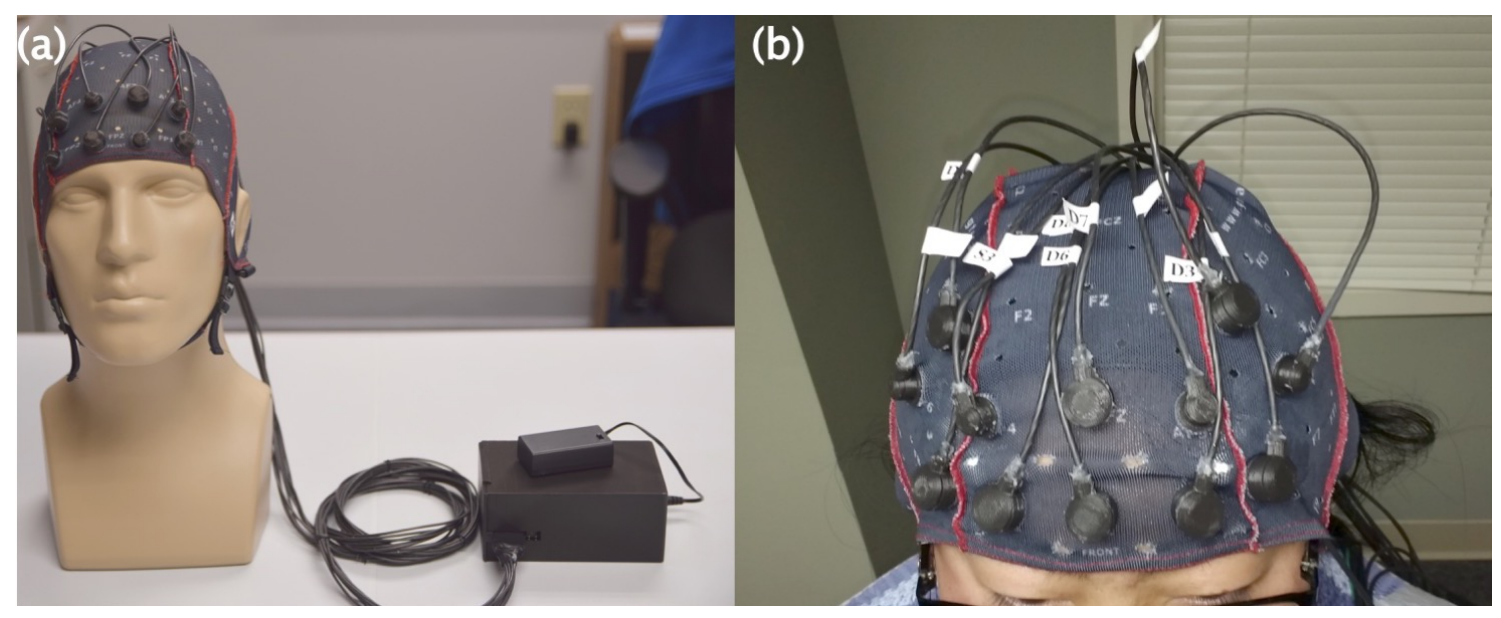

Figure 35. (a) WearLight fNIRS system, the system consists of an optode cap and acontrol box connected to a battery and (b) participant wearing the fNIRS montage cap having four source and eight detector optodes designed for prefrontal cortex imaging in our n-back WM task.

Different neuroimaging studies identified that neurons in the prefrontal cortex (PFC) brain areas are involved in processes necessary for WM related tasks [5, $6,7,8,9,10]$. WM task also demonstrated activation of the bilateral network consisting of dorsolateral prefrontal cortex (DLPFC) and ventrolateral prefrontal cortex (VLPFC), lateral premotor cortex, frontal poles, medial premotor cortices, dorsal cingulate and medial and lateral posterior parietal cortices [11, 12]..

One of the well-established experimental method in the neuroscience and cognitive psychology is to use of n-back memory task to manipulate WM levels [13]. In the n-back experiment, WM load increases with the increasing number of items (n) to be memorized, until the task-specific participant's WM capacity is reached. The increasing WM load also degrades the task performance such as accuracy and reaction time and increases physiological arousal and heart rate [14].

Some studies tried to estimate the level of WM loads by measuring changes in the physiological parameters such as heart rate or skin conductance $[15,16]$. However, deducing WM load from these peripheral physiological measurements tends to be challenging as they are largely influenced by emotional [17] and physiological 
conditions [18].

Direct measurement of the electrical activity of the neurons using electroencephalography (EEG) has been a strong candidate to assess cognitive load. Different experimental studies have shown that increased WM load correlated with the decreased power in alpha activity $(8-15 \mathrm{~Hz})$, whereas increased beta $(16-31 \mathrm{~Hz})$ and theta (4-7 Hz) activity at the PFC area of the brain [19, 20,21]. Although EEG has many advantages in imaging brain, including high temporal resolution and portability, it suffers from low spatial resolution. Additionally, it demands longer subject preparation time and susceptible to motion artifact.

An indirect method of imaging the neural activity is by measuring changes of regional hemoglobin in the cerebral blood of the brain. This method is based on the fact that the area of high neuronal activation increases the oxygen consumption and thereby increases blood supply to that active brain region, ensuring supply of oxygenated hemoglobin [22]. The brain's hemodynamic response in the working memory tasks has been widely studied using functional Magnetic Resonance Imaging (fMRI) neuroimaging technique [11]. However, fMRI is not applicable to all subject types, and confines the subject in a small space and has a high operating cost.

Another modality based on imaging same brain's hemodynamic response is functional near-infrared spectroscopy (fNIRS) that offers a viable alternative for cognitive state monitoring. Since the first demonstration of fNIRS in the year 1977 [23], fNIRS has been increasingly getting popularity as a neuroimaging tool for its several benefits. It provides similar blood oxygenation level dependent (BOLD) functional image of brain [24] as fMRI by noninvasively imaging temporal and spatial changes in oxygenated $\left(\mathrm{HbO}_{2}\right)$ and deoxygenated $(\mathrm{Hb})$ hemoglobin concentrations [25]. In addition, fNIRS has a high temporal resolution, safe and bears 
low operating cost, and most importantly a portable fNIRS system can allow brain imaging in a naturalistic environment. Hence, it's suitable to perform neuroimaging studies with a large subject population, including psychiatric patients and children, outside of the clinical environment. Moreover, the subject preparation time can be significantly less $(\sim 1 \mathrm{~min})$.

There are various studies where fNIRS and n-back task have been used to assess the working memory load. For intense, Schecklmann et al. used an fNIRS system (ETG-4000 optical topography system, Hitachi Medical Co., Japan) to image PFC of nineteen childrens with attention-deficit/hyperactivity disorder (ADHD) [26]. They found significant differences in WM performance for three different task groups, object visual (OWM), spatial visual working memory (SWM) and control condition $(\mathrm{CON})$.

Another study reported significant hemodynamic response difference in two groups in the WM n-back task, subject with traumatic brain injury (TBI) and healthy controls (HC) groups (six TBI and eleven HC subjects) [27]. This study used an fNIRS system developed in the Drexel University (Philadelphia, PA) consisting of a headpiece that holds four light sources and 10 detectors and a control box to image.

Fishburn et al. used a CW5 imaging system (TechEn, Inc., Milford, MA) [28]. They arranged 12 sources and 29 detectors of the system into 5 probes to image bilateral prefrontal, bilateral partial and frontal lobe of the brain of sixteen young participants. They found increased brain activation and functional connectivity with the increasing memory loads in the n-back task.

Also, fNIRS has been utilized to measure and assess the mental workload of the operators in the stressful critical jobs such as air traffic control (ATC), military command and control, unmanned vehicle and aircraft operation. Some studies used 
the fNIRS system from fNIR Devices LLC (Potomac, MD; www.fnirdevices.com) to image PFC. This fNIRS device has a headband with four LED light sources and ten light detectors (optodes) creating total of 16 fNIRS data channels. Using this system, Ayaz et al. imaged the $\mathrm{HbO}_{2}$ level in the PFC of ATC operators in the flight simulator [29]. In this study three different levels of working memory load correspond to the ATC communication tasks were distinguished. Harrison et al. used this fNIRS device to access learning and workload of ATC operator and found increased mean oxygenation level due to increasing air traffic density [30].

Another study used 40-channel fNIRS device (NIRsport from NIRx, Germany) to record hemodynamic activity from fifteen healthy adults during visuospatial WM task [31]. They found that in the visuospatial WM system, the cortical activation and functional coherence in the frontal-parietal cortices are dissociated.

Some studies suggested the integration of momentary WM load measurement of the car driver in the car automation systems so that the systems are adaptive to the driver's cognitive workload. Tsunashima et al. used an fNIRS instrument (OMM-3000, Shimadzu Corporation, Japan) to study the hemodynamic activity of four participants in the driving simulator with and without adaptive cruise control (ACC) [32]. They found that ACC resulted in less frontal lobe activations while driving

In this paper, we have performed experimental studies to access four different levels of working memory loads using our portable fNIRS system, WearLight as shown in figure 35 (a). We created an n-back experimental protocol that has 16 trials in one run. The four different n-back conditions (0 to 3-back) are pseudorandomized in the 16 trials. We recruited 13 participants to image prefrontal cortex areas of the brain while participants performing the n-back task. Figure 35 (b) shows a participant wearing the fNIRS montage cap with four source op- 
todes and eight detector optodes used in the experiment. Significant differences in brain's hemodynamic response have been observed in the four task difficulty levels. The incremental brain's hemodynamic activity with the task difficulty depicts the four working memory loads induced by the n-back task. The results proved that portable WearLight fNIRS system is instrumental in assessing and distinguishing cognitive load. The battery operated system is wireless and wearable, hence it can be implemented for the development of BCI system applicable in the naturalistic environment.

\subsection{Materials and Methods}

\subsubsection{Functional Near-infrared Spectroscopy (fNIRS)}

Functional near-infrared spectroscopy (fNIRS) is an emerging noninvasive neuroimaging technique that spatially and temporally maps hemodynamic response, oxy-hemoglobin $\left(\mathrm{HbO}_{2}\right)$ and deoxy-hemoglobin $(\mathrm{Hb})$ changes, of the brain in order

to image the human brain functions. The fNIRS sensor cap (montage) consists of multi-wavelength near-infrared (NIR) light sources and light detectors (they are commonly denoted as optodes) arranged in an experiment-specific order. Usually, the light sources and detectors are 25-40 $\mathrm{mm}$ apart. The NIR light sources are sequentially turned on and propagated light through the tissue is absorbed by chromophores (dominantly by $\mathrm{HbO}_{2}$ and $\mathrm{Hb}$ ) on the cortical area of the brain. The light absorption is wavelength dependent as shown in the figure fig:spectra. Hence, performing continuous spectroscopic measurements of the back-reflected diffuse light from the cortical surface of the brain using the detectors attached to the montage cap, it is possible to estimate the $\Delta \mathrm{HbO}_{2}$ and $\Delta \mathrm{Hb}$ over time. $\mathrm{A}$ source-detector (SD) pair, mostly sensitive to the brain activity around the midpoint of the SD pair, creates an fNIRS channel. Figure 36 shows a source and a detector at $d \mathrm{~mm}$ apart (SD pair) and the corresponding fNIRS channel probing 
the brain. By placing a number of sources and detectors on the head surface and performing time multiplexed high-speed measurements from all the SDs, the realtime imaging of the brain activity is possible in this technique. Also, frequency and/or spatial multiplexing can be implemented to further improve the imaging frame rate.

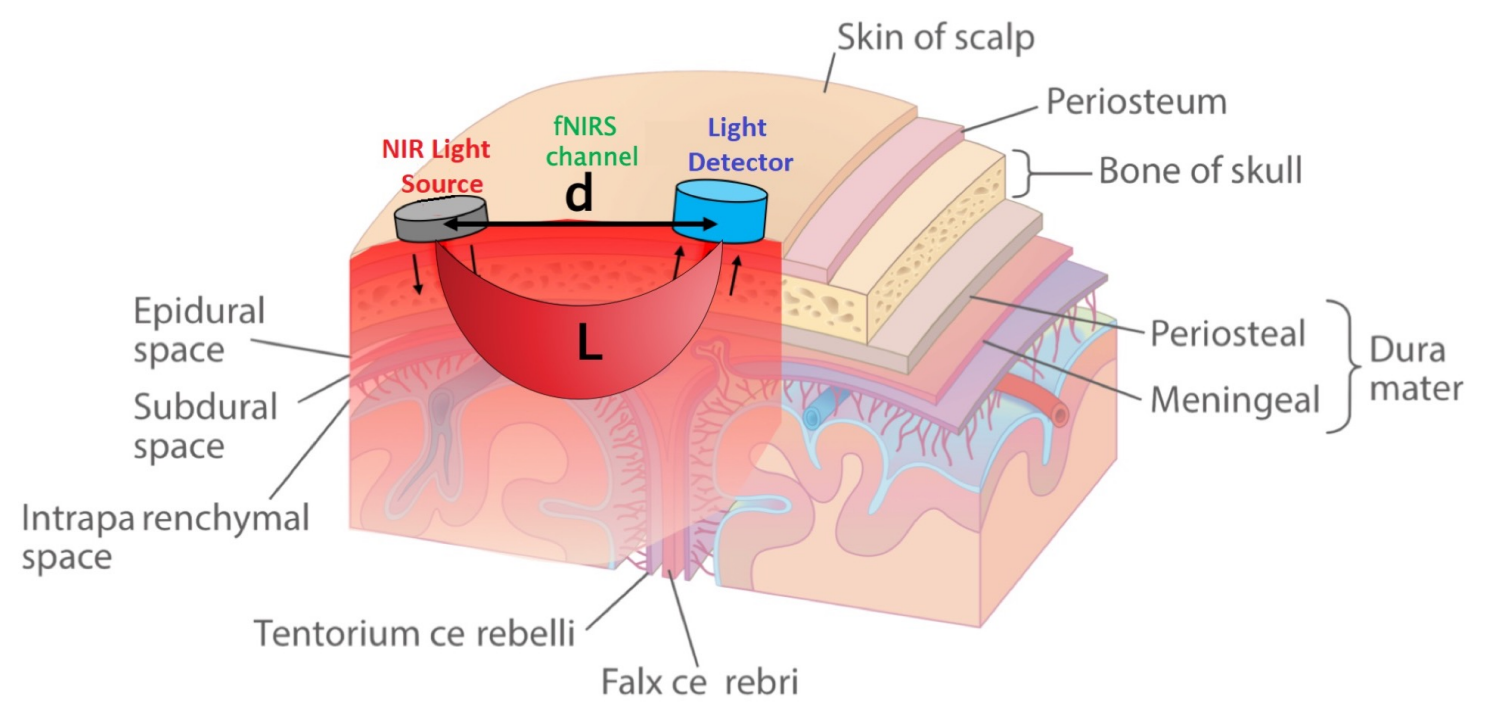

Figure 36. Sketch of NIR light propagation from a source to a detector through different tissue layers of a brain forming an fNIRS channel. Source and detector separating distance $(d)$ is related to optical path length $(L)$ by $L=D P F . d$.

Light propagation through the biological tissue can be accurately modeled using radiative transfer equation (RTE) [34] or a simplified version of RTE, namely, the diffusion equation (DE) [35]. DE can be further simplified to Modified BeerLambert Law (MBLL) under various assumptions such as (1) tissue is homogeneous, (2) scattering is constant and (3) optical path length is known. In fNIRS brain imaging, changes in chromophore concentrations $(\Delta C)$ due to brain activities are most widely calculated using MBLL for its simplicity and computational efficiency. We use MBLL to calculate changes in blood chromophores concentration,

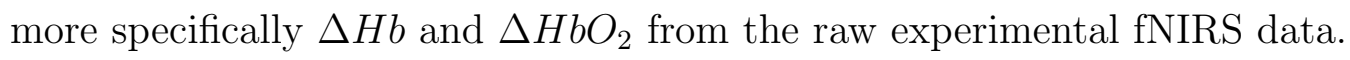

In a purely absorbing medium, the transmitted light $(I)$ can be expressed in 


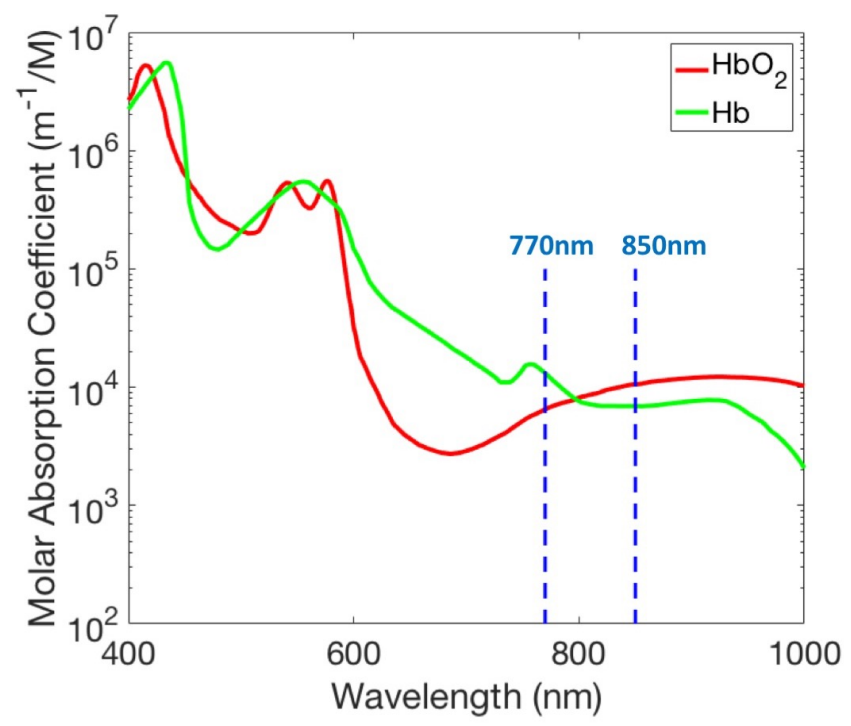

Figure 37. Absorption spectra of oxy-hemoglobin $\left(\mathrm{HbO}_{2}\right)$ and deoxy-hemoglobin $(\mathrm{Hb})$ of tissue, represented in terms of molar absorption coefficients. Data were taken from OMLC [33]. The wavelengths of the LEDs used for our fNIRS system are indicated by the vertical dashed line.

terms of input light $\left(I_{o}\right)$ as,

$$
I=I_{o} e^{-\mu_{a} L}
$$

where $\mu_{a}$ is the absorption coefficient of the homogeneous medium and $L$ is the optical path length. Absorption coefficient is related to the concentration of the absorbing compound $(C)$ and extinction coefficient $(\varepsilon)$ as,

$$
\mu_{a}=\varepsilon C
$$

From Equ. 3 and Equ 4, the absorption of light in terms of optical density (OD) can be formulated as,

$$
A=O D=\ln \left(\frac{I_{o}}{I}\right)=\varepsilon C L
$$

For the $N$ number of absorbing compounds present in the medium, the absorption of light given by, 

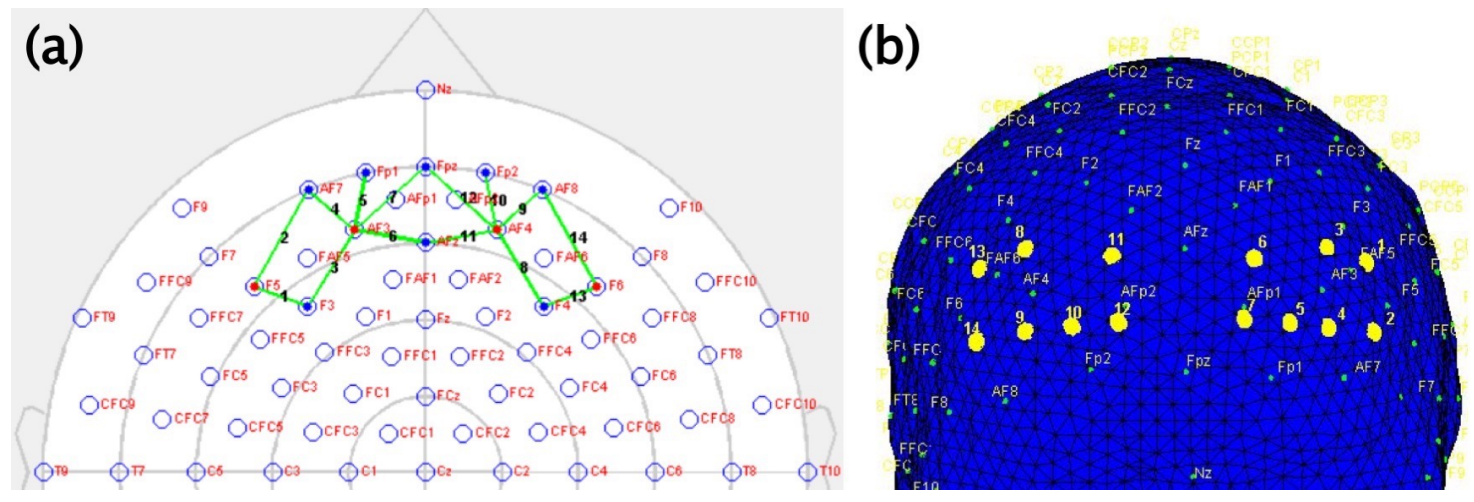

Figure 38. The fNIRS montage used in the experiment. (a) LEDs (red), detectors (blue) and the fNIRS channels (green line) location on a standard 10-20 EEG system. (b) fNIRS channels (yellow label) onto the head model showing the coverage on the prefrontal areas. Figures are generated using nirsLAB software.

$$
A=\left[\varepsilon_{1} C_{1}+\varepsilon_{2} C_{2}+\varepsilon_{3} C_{3}+\ldots+\varepsilon_{N} C_{N}\right]
$$

Similar to the absorption case, if the medium has only one scattering compound (for simplicity) with scattering coefficient $\mu_{s}$, the transmitted light intensity $I$ can be written as,

$$
I=I_{o} e^{-\mu_{s} L}
$$

The attenuation of light while propagating through the tissue is resulted from the combined effect of multiple scattering of light and absorption. Hence, both of these effects should be accounted to accurately quantify changes in concentration of chromophores. The attenuation of light accounting both the absorption and multiple scattering can be expressed by MBLL as follows,

$$
A=\ln \left(\frac{I_{o}}{I}\right)=\varepsilon C L+G
$$

where $G$ is the attenuation of light due to scattering which is assumed to be constant as the contribution from the changes in hemoglobin concentration due to 
the brain activities to scattering is negligible.

The mean path length $(L)$ of light in the highly scattering tissue is larger than the source-detector geometrical path length (d) as shown in the figure 36 . The differential path length factor (DPF) is multiplied to $d$ in order to correct $L$.

$$
L=D P F \cdot d
$$

Hence, by measuring $A$ at least at two wavelengths, the relative changes in two chromophores concentration $(\Delta C)$ in time can be estimated from the relative changes in attenuation $(\Delta A)$

$$
\Delta A=\varepsilon \Delta C \cdot d . D P F
$$

The fNIRS brain imaging is based on calculating changes in hemoglobin concentrations $\left(\Delta C_{\mathrm{Hb}}\right.$ and $\left.\Delta C_{\mathrm{HbO}_{2}}\right)$ from the measured attenuation changes at two wavelengths ( $\Delta A^{\lambda 1}$ and $\Delta A^{\lambda 2}$ ). Also, including wavelength dependency of $D P F$ we get,

$$
\left[\begin{array}{c}
\Delta C_{H b} \\
\Delta C_{H b O_{2}}
\end{array}\right]=\left[\begin{array}{ll}
\varepsilon_{H b}^{\lambda 1} / d . D P F^{\lambda 1} & \varepsilon_{H b O_{2}}^{\lambda 1} / d . D P F^{\lambda 1} \\
\varepsilon_{H b}^{\lambda 2} / d . D P F^{\lambda 2} & \varepsilon_{H b O_{2}}^{\lambda 2} / d . D P F^{\lambda 2}
\end{array}\right]\left[\begin{array}{c}
\Delta A^{\lambda 1} \\
\Delta A^{\lambda 2}
\end{array}\right]
$$

\subsubsection{N-back memory task}

In the n-back task, subjects are sequentially presented items (letters, number or patterns, etc.) and subjects are engaged in remembering the previous $n$ set of rapidly sequentially flashing items at any moment of time. The subjects are asked to respond when the current item (stimulus) is the same as the $n^{\text {th }}$ item before the current item. The task difficulty level can be increased by increasing $n$, as greater the $n$ more the items subjects need to remember from the continuously shifting sequence of items. Thus, WM load increases with $n$. The subjects respond 
(a)

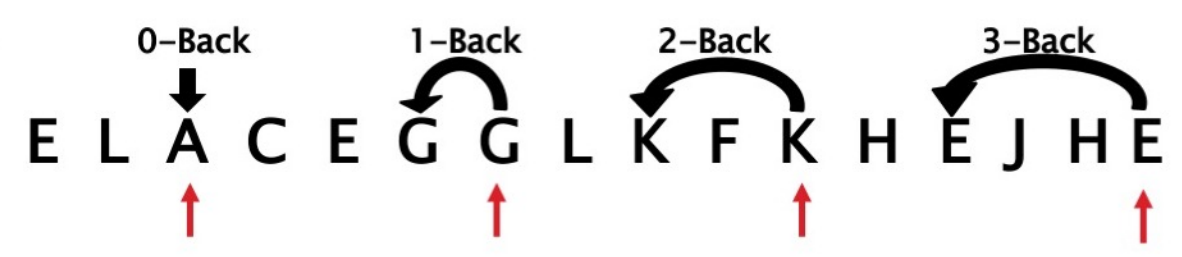

(b)

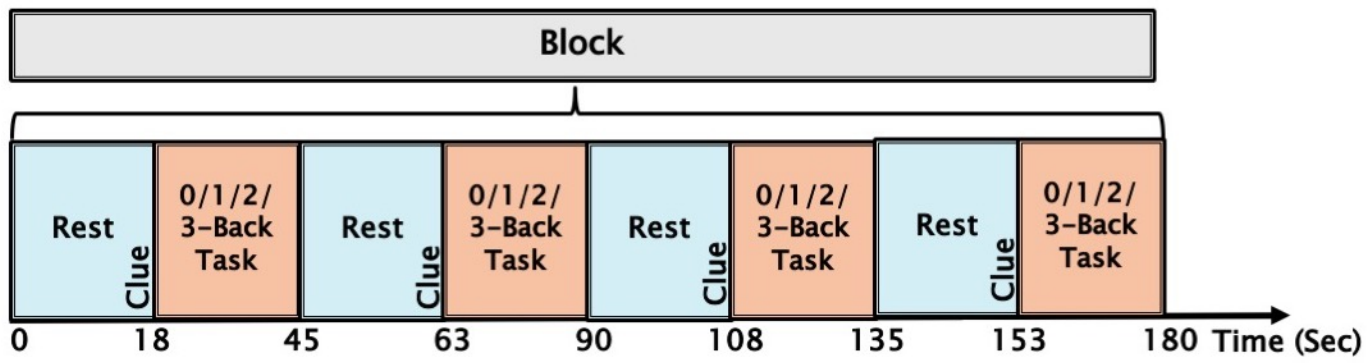

(c)

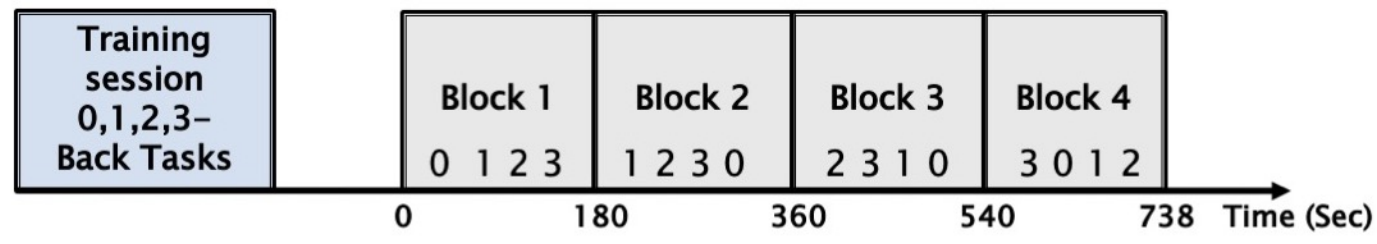

Figure 39. Experimental paradigm. (a) concept of 0, 1, 2, and 3-back tasks, (b) a task block and each task block has 0,1,2 and 3-back conditions and (c) four task blocks each with four n-back pseudo-randomized conditions. A complete run takes about 738 seconds.

using an input device such a keypad, switch or mouse. The subjects' responses and the brain signals are time synchronized with the task. Hence, the subjects' performance can be measured later by looking at the number of targets missed, wrong reactions and reaction time along with the WM load derived from the brain signal.

\subsubsection{Experimental setup}

We recorded the hemodynamic response of the prefrontal cortex while participants performed n-back working memory task using our experimental protocol explained in the Section 5.3.4. To record the fNIRS signal, we used a portable continuous wave fNIRS system, WearLight, developed in the University of Rhode Island, Kingston, RI, USA [36]. We designed a montage with four LED light 
sources and eight photodiode detectors. Fig. 38 (a) shows the source and detector location in a standard 10-20 EEG system. The source-detector arrangement formed a total of 14 active fNIRS channels (green line in Fig. 38 (a)) covering the prefrontal cortex. Fig. 38 (b) presents the fNIRS channel location on the head model. The LED light sources emit NIR light with peak wavelengths of 770 $\mathrm{nm}$ and $850 \mathrm{~nm}$. The WearLight system is battery operated and montage cap is connected to the portable control box using a flexible cable as shown in Fig. 35 (a). A computer connected to the control box collected fNIRS data and stored for processing.

We used a MatLab based graphical user interface (GUI) software in order to control and collect data from the WearLight system. The GUI software plots fNIRS signals in real-time that allows us for signal quality check through visual inspection. Prior to recording, we improved the optode-scalp coupling by adjusting the optodes on the scalp to achieve best possible fNIRS signal. We used another computer for the n-back stimulus presentation. We time synchronized the fNIRS data recording computer with the n-back stimulus presenting computer using trigger signals. After every experimental session, fNIRS data and the user response data files were securely saved in a common folder for data processing following the data processing strategy explained in Section 5.3.6.

\subsubsection{Experimental paradigm}

In our experiment, participants were asked to comfortably sit in front of a computer screen. The screen presented a stream of English alphabet letters and participants required to compare the currently presented letter with the letter that occurred $n$ steps back. When the letter matched the participant responded by pressing "0" button on the keypad with their dominant hand to identify the targeted stimulus. BCI2000 [37] controlled the task presentation and recorded 
the user response. The button pressing data, stimulus, and fNIRS data were time synchronized in the experiment to help evaluate participants' performance, engagement level and hemodynamic response with respect to the task. We had four different n-back tasks levels 0, 1, 2, and 3-back as shown in Fig. 39 (a). In the 0-back task condition, participants were asked to respond to a single predefined target letter " $A$ ". In the 1-back task condition, the target is the letter matching just the previous letter. In the 2-back and 3-back task conditions, the targets were the letters that were identical to the letter presented two and three letters before the current letter respectively.

We have all total of 16 trials in the experiment. The 16 trials were arranged in 4 different blocks [Fig. 39 (c)]. Each block has 0, 1,2 and 3-back task conditions Fig. 39 (b), but the order of these four conditions in the blocks was pseudo-randomized.

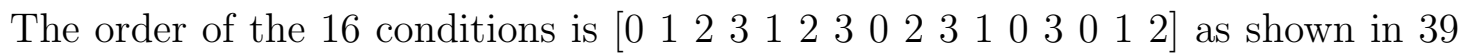
(c). Each trial contained 3 target and 6 non-target letters. The location of the target and non-target letters were also randomized in all the 16 series of letters (16 trials). Every letter was displayed for 500 milliseconds and the screen was left blank for 2.5 seconds. Thus in 3 seconds, a new letter was presented resulting in each trial duration of 27 seconds. There were 18 seconds of rest period between the trials including 3 seconds for clue to display information about which task ( 0 , 1,2 , or 3 -back) was about to start. In the rest period, participants were asked to relax so that hemodynamic activity returns to baseline. We first performed a training session before the actual recording, where we explained the tasks and participants performed a short practice session. The entire recording time was about 738 seconds as shown in the time axis in Fig. 39 (c). 


\subsubsection{Participants}

This study included healthy 13 right-handed participants (2 female and 11 male), aged 22-27 (mean age $=22$ ), and with a mean of 15 years of formal education. The participants were recruited from the student body of the University of Rhode Island, RI, USA. The participants were informed prior to the experiment and gave written consent. In addition, participants filled out a screening questionnaire. Inclusion criteria included no medical record of head trauma or neurological illness, no prescribed medication at the time of the study, fluency in English, and good eyesight. The study was approved by the University of Rhode Island Institutional Review Board.

\subsubsection{Data analysis}

The raw experimental data collected from the brain using any fNIRS system is influenced by various artifacts, for example, physiological noise, movement artifact, electrical and optical noise. Physiological signals such as cardiac artifacts, respiration rate, Meyer waves are superimposed in the fNIRS signal. Another common artifact is due to the movement of participant that alters the optode-scalp coupling resulting spikes in the fNIRS signal. We processed the raw fNIRS data using our MatLab based fNIRS data processing software. We measured the SNR and also performed a preliminary visual inspection of the data prior to signal processing. We used a band-pass filter (cutoff frequencies: $0.01 \mathrm{~Hz}$ and $0.2 \mathrm{~Hz}$ ) to reduce artifacts and to retain the low-frequency fNIRS signal. We used molar extinction coefficient values of $\mathrm{HbO}_{2}$ and $\mathrm{Hb}$ corresponding to wavelengths of 770 and $850 \mathrm{~nm}$, respectively from literature [33]. The MBLL [Equ. 11] was applied to convert the data from voltage $(\mathrm{mV})$ to relative concentration change $\triangle \mathrm{HbO}_{2}$ and $\Delta H b$. Then we spatially mapped the fNIRS channel data on the brain for the topographic visualization. 

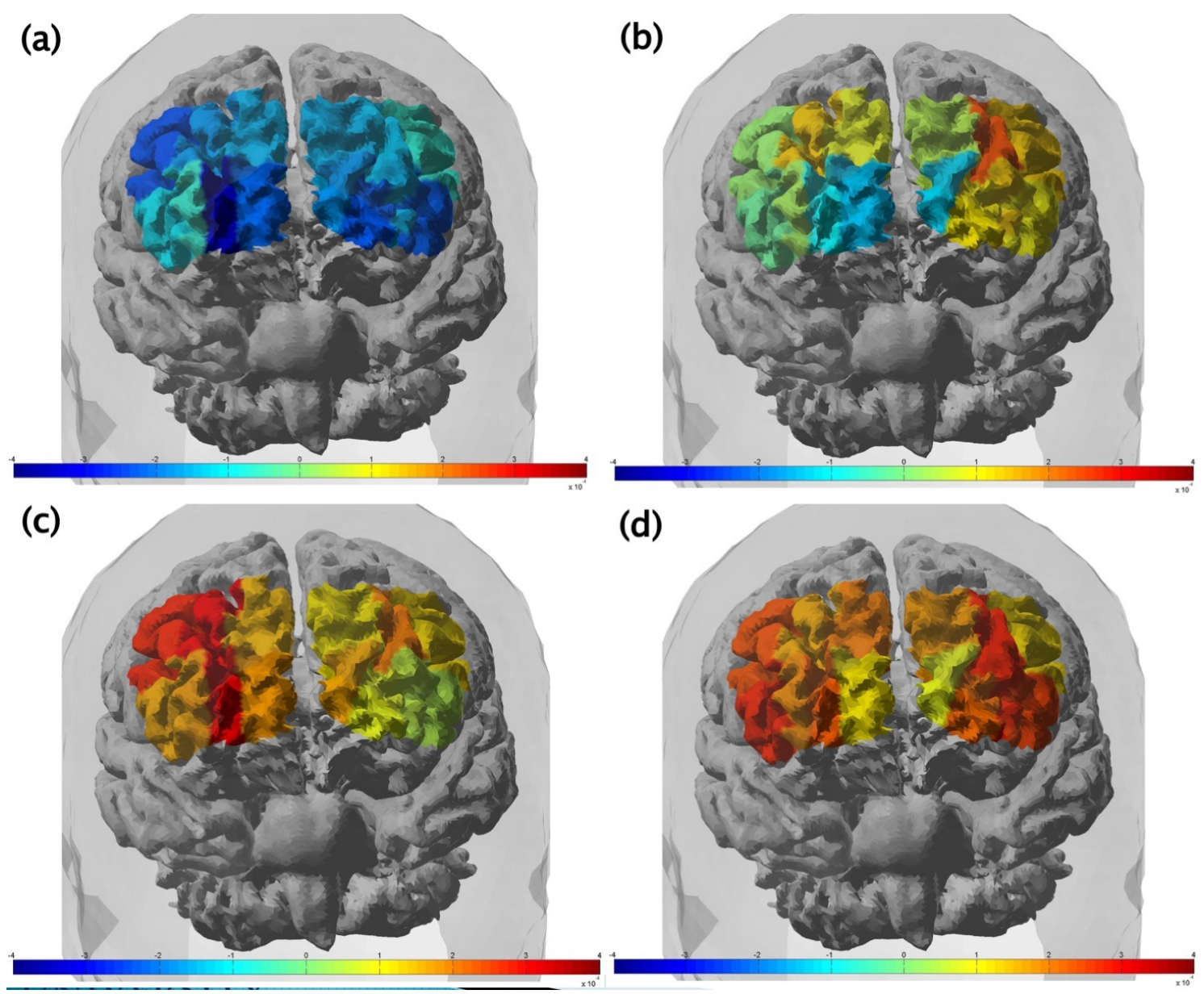

Figure 40. Increasing oxyhemoglobin $\left(\mathrm{HbO}_{2}\right)$ responses with difficulty levels in the n-back tasks, (a) 0-back, (b) 1-back, (c) 2-back and (d) 3-back

\subsection{Results}

\subsubsection{Task performance}

We analyzed the participants' task performance data to verify that the participants experienced different difficulty levels in the different n-back conditions. We also conducted a feedback session after each experiment to note the participants' experience in the experiment. There were $0 \%$ targets missed by the participants in the 0-back task. While the percentage of targets missed increased from $1 \%$ on average for the 1-back condition to $6 \%$ for 2 -back to $13 \%$ for the 3 -back task. We also evaluated the number of wrong reactions when participants incorrectly identified a non-target letter as a target by pressing the " 0 " key. The amount of 


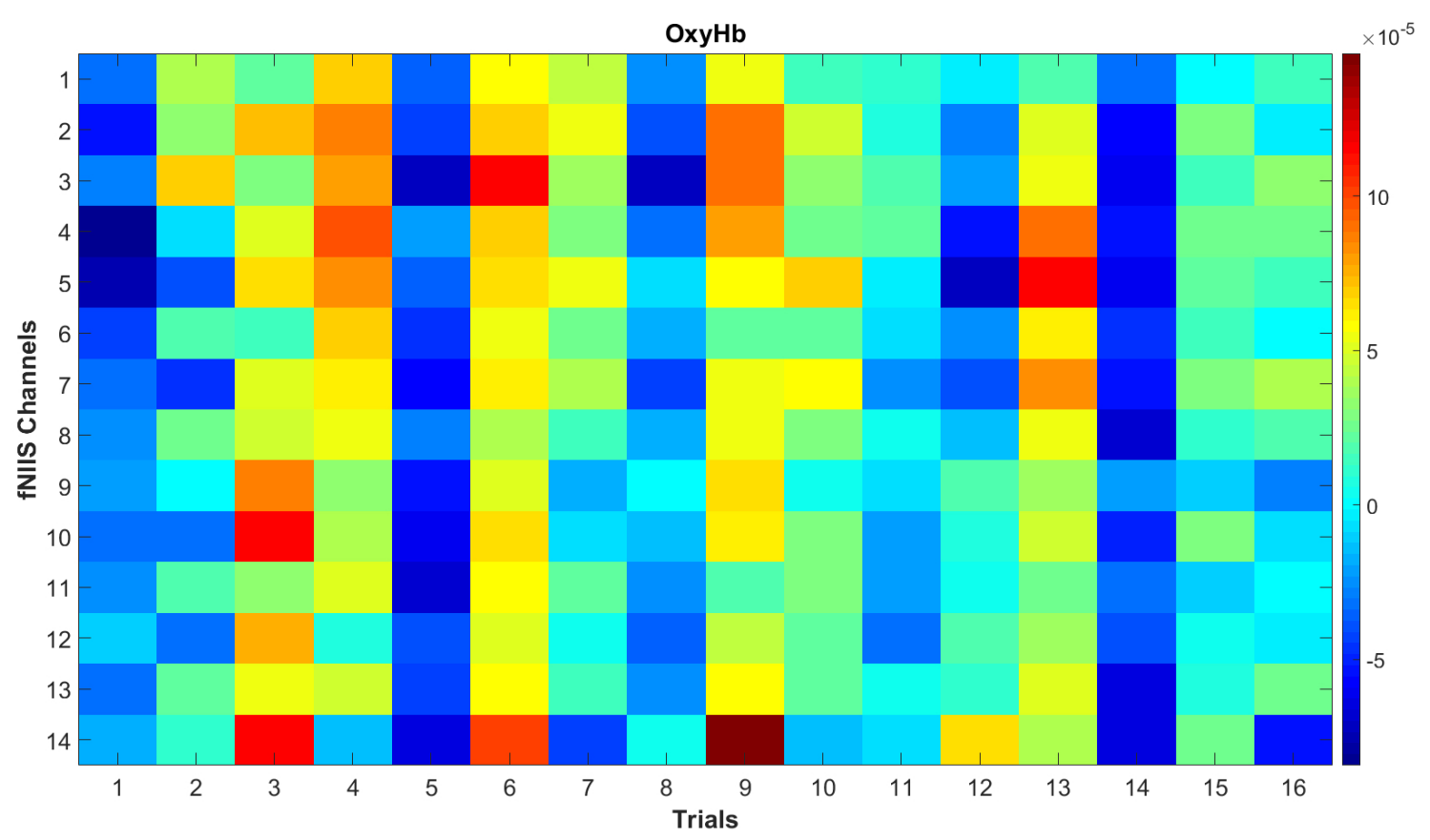

Figure 41. Oxyhemoglobin mean response over the entire participants group and in trials. Rows are the 14 fNIRS channels and column are the 16 n-back trials. The channel number are the same as in the Fig. 38.

the wrong reactions for $0,1,2$ and 3-back tasks were on average $0,0,2.8$ to 4.5 respectively.

The participants were from the Bachelor of Science program and they went through a short n-back training session before the actual session. This might resulted in a better task performance compared to the other reported studies. However, the differences in targets missed and wrong reactions between $0,1,2$ and 3-back were noticed. These analyses and subjective data in the feedback session clearly verified that the four n-back task conditions had significantly different difficulty levels and that demanded four different mental workload levels.

\subsubsection{Brain hemodynamic response}

The data analysis was performed after the raw data was converted to oxyhemoglobin $\left(\mathrm{HbO}_{2}\right)$ and deoxy-hemoglobin $(\mathrm{Hb})$ changes using data analysis strategy and MBLL explained in section 5.3.6. Figure 40 shows topographic images of 


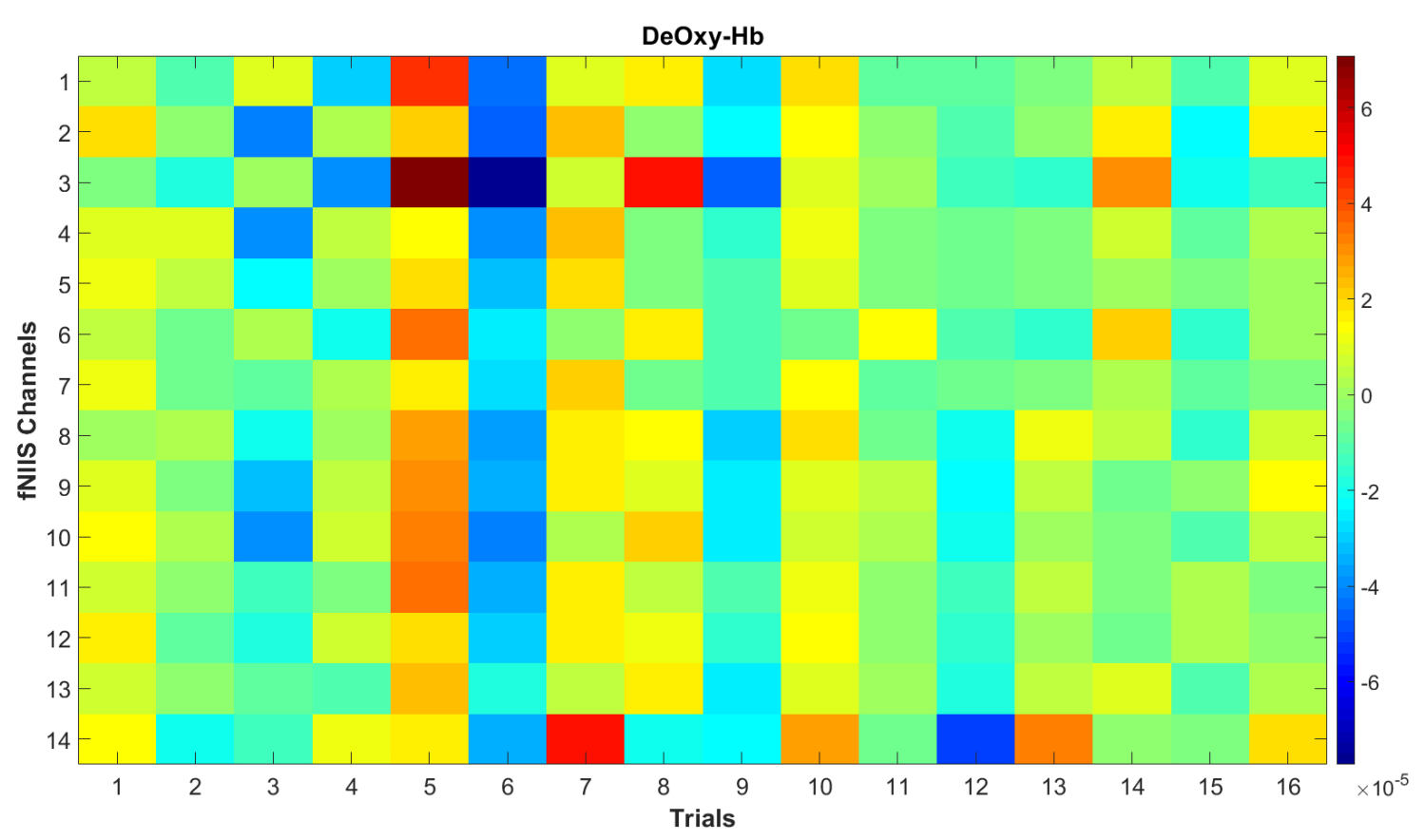

Figure 42. De-oxyhemoglobin mean response over the entire participants group and in trials. Rows are the 14 fNIRS channels and column are the 16 n-back trials. The channel number are the same as in the Fig. 38.

$\mathrm{HbO}_{2}$ values in the four n-back tasks of a participant. We observed that $\mathrm{HbO}_{2}$ variation over both the hemispheres in the PFC and the $\mathrm{HbO}_{2}$ response increased with the difficulty level of n-back task.

After obtaining time course of hemodynamic activities of all the participants, we evaluated mean response over all the participants and within each trial to identify areas on the PFC of the brain which showed significant hemodynamic response and WM load levels due to the various n-back task stimulations. Figure 41 and 42 present the mean $\mathrm{HbO}_{2}$ and $\mathrm{Hb}$ responses at group level in the 16 trials. The $\mathrm{HbO}_{2}$ level reached the peak in the 3-back conditions (trial 4, 7, and 10). Also, overall slightly less response was observed in the 2-back conditions (trial 3, 6 , and 9), whereas in the 1 -back trial blocks $(2,5,11$ and 15), it was minimum and 0-back condition (trial 1, 8, 12 and 14) response is close to baseline level. On the other hand, we noticed the $\mathrm{Hb}$ response is opposite that of $\mathrm{HbO}_{2}$ response. It 
decreased with increasing of $\mathrm{HbO}_{2}$. The decreasing order of $\mathrm{Hb}$ response for the four conditions is 3, 2, 1 and 0-back as evidenced in Fig 42. The distribution of the four n-back tasks in the trail sequence and the duration of all the trials together resulted in more hemodynamic activation between trial number 3 to 9 out of those 16 trials. We also observed consistent hemodynamic activities over both left and right hemispheres during all the trials. However, in general, the spatial distribution of the channels (fNIRS channel 1 to 7 ) showed slightly higher left PFC activation than the right one.

\subsection{Discussion and Conclusion}

Many neuroimaging studies provided evidence that the prefrontal cortex is gradually activated more when working memory load is increased. Our results confirmed PFC activation with WM demand. Moreover, by comparing hemodynamic responses of the brain in the 0-back, 1-back, 2-back, and 3-back conditions, we found an increase in PFC activation with $n$, the WM task difficulty level. The task difficulty induced the mental effort and increased the WM load in the PFC. Both the hemisphere have shown the same trend; however, a slightly stronger activation was observed over the left cortex. The presented investigation by our portable fNIRS system supports results from other studies, showing stronger hemodynamic response with incremental WM task difficulty.

In conclusion, the study successfully demonstrated the capability of WearLight portable fNIRS system to access and characterize the PFC brain activation patterns risen by WM load. Portable fNIRS is a promising technology for the noninvasive brain imaging in the naturalistic environment that can lead to the development of widely applicable BCI system. Future studies on more advanced techniques such as Machine Learning (ML) will be performed for the development of such a BCI system. 


\section{Acknowledgment}

This material is based upon work supported by the National Science Foundation under Grant No. 1539068. The authors would also like to thank Joanne Hall, Lynn McCane and Shunan Li for their assistance.

\section{List of References}

[1] A. Baddeley, "Working memory: looking back and looking forward," Nature Reviews Neuroscience, vol. 4, no. 10, pp. 829-839, 2003. [Online]. Available: https://doi.org/10.1038/nrn1201

[2] J. M. Fuster, Memory in the cerebral cortex: An empirical approach to neural networks in the human and nonhuman primate. Cambridge, MA, US: The MIT Press, 1995.

[3] P. S. Goldman-Rakic, Architecture of the prefrontal cortex and the central executive. New York, NY, US: New York Academy of Sciences, 1995, pp. 71-83.

[4] G. A. Miller, "The magical number seven, plus or minus two: some limits on our capacity for processing information." Psychological Review, vol. 63, no. 2, pp. 81-97, 1956.

[5] M. D'Esposito, B. Postle, D. Ballard, and J. Lease, "Maintenance versus manipulation of information held in working memory: An event-related fmri study," Brain and Cognition, vol. 41, no. 1, pp. 66 - 86, 1999. [Online]. Available: http://www.sciencedirect.com/science/article/pii/ S0278262699910965

[6] B. Spitzer, D. Goltz, E. Wacker, R. Auksztulewicz, and F. Blankenburg, "Maintenance and manipulation of somatosensory information in ventrolateral prefrontal cortex," Human Brain Mapping, vol. 35, no. 5, pp. 2412-2423, 2019/06/13 2014. [Online]. Available: https://doi.org/10.1002/hbm.22337

[7] E. E. Smith and J. Jonides, "Working memory: A view from neuroimaging," Cognitive Psychology, vol. 33, no. 1, pp. 5 - 42, 1997. [Online]. Available: http://www.sciencedirect.com/science/article/pii/S0010028597906587

[8] T. Klingberg, "Development of a superior frontal-intraparietal network for visuo-spatial working memory," Neuropsychologia, vol. 44, no. 11, pp. 2171 - 2177, 2006, advances in Developmental Cognitive Neuroscience. [Online]. Available: http://www.sciencedirect.com/science/article/ pii/S0028393205003830 
[9] S. Funahashi, "Prefrontal cortex and working memory processes," Neuroscience, vol. 139, no. 1, pp. 251 - 261, 2006. [Online]. Available: http://www.sciencedirect.com/science/article/pii/S030645220500744X

[10] L. Pessoa and L. G. Ungerleider, Top-Down Mechanisms for Working Memory and Attentional Processes. Cambridge, MA, US: MIT Press, 2004, pp. 919 930.

[11] A. M. Owen, K. M. McMillan, A. R. Laird, and E. Bullmore, "N-back working memory paradigm: A meta-analysis of normative functional neuroimaging studies," Human brain mapping, vol. 25, no. 1, pp. 46-59, 2005.

[12] S. Funahashi, C. J. Bruce, and P. S. Goldman-Rakic, "Mnemonic coding of visual space in the monkey's dorsolateral prefrontal cortex," Journal of Neurophysiology, vol. 61, no. 2, pp. 331-349, 1989, pMID: 2918358. [Online]. Available: https://doi.org/10.1152/jn.1989.61.2.331

[13] W. K. Kirchner, "Age differences in short-term retention of rapidly changing information." Journal of Experimental Psychology, vol. 55, no. 4, pp. 352-358, 1958.

[14] R. W. Backs and K. A. Seljos, "Metabolic and cardiorespiratory measures of mental effort: the effects of level of difficulty in a working memory task," International Journal of Psychophysiology, vol. 16, no. 1, pp. 57-68, 1994. [Online]. Available: http://www.sciencedirect.com/science/article/pii/ 0167876094900426

[15] T. M. Gable, A. L. Kun, B. N. Walker, and R. J. Winton, "Comparing heart rate and pupil size as objective measures of workload in the driving context: Initial look," in Adjunct Proceedings of the 7th International Conference on Automotive User Interfaces and Interactive Vehicular Applications, ser. AutomotiveUI '15. New York, NY, USA: ACM, 2015, pp. 20-25. [Online]. Available: http://doi.acm.org/10.1145/2809730.2809745

[16] E. T. Solovey, M. Zec, E. A. Garcia Perez, B. Reimer, and B. Mehler, "Classifying driver workload using physiological and driving performance data: Two field studies," in Proceedings of the SIGCHI Conference on Human Factors in Computing Systems, ser. CHI '14. New York, NY, USA: ACM, 2014, pp. 4057-4066. [Online]. Available: http://doi.acm.org/10.1145/2556288.2557068

[17] D. Sander, D. Grandjean, and K. R. Scherer, "A systems approach to appraisal mechanisms in emotion," Neural Networks, vol. 18, no. 4, pp. 317 - 352, 2005, emotion and Brain. [Online]. Available: http: //www.sciencedirect.com/science/article/pii/S0893608005000341 
[18] K. A. Brookhuis and D. de Waard, "Monitoring drivers'mental workload in driving simulators using physiological measures," Accident Analysis 63 Prevention, vol. 42, no. 3, pp. 898-903, 2010. [Online]. Available: http://www.sciencedirect.com/science/article/pii/S000145750900116X

[19] W. Klimesch, "Eeg alpha and theta oscillations reflect cognitive and memory performance: a review and analysis," Brain Research Reviews, vol. 29, no. 2, pp. 169 - 195, 1999. [Online]. Available: http: //www.sciencedirect.com/science/article/pii/S0165017398000563

[20] A. Gevins, M. E. Smith, L. McEvoy, and D. Yu, "High-resolution eeg mapping of cortical activation related to working memory: effects of task difficulty, type of processing, and practice." Cerebral Cortex, vol. 7, no. 4, pp. 374-385, 6/14/2019 1997. [Online]. Available: https://doi.org/10.1093/cercor/7.4.374

[21] J. B. Brookings, G. F. Wilson, and C. R. Swain, "Psychophysiological responses to changes in workload during simulated air traffic control," Biological Psychology, vol. 42, no. 3, pp. 361 - 377, 1996, psychophysiology of Workload. [Online]. Available: http://www.sciencedirect.com/science/ article/pii/0301051195051678

[22] M. Banaji, A. Mallet, C. E. Elwell, P. Nicholls, and C. E. Cooper, "A model of brain circulation and metabolism: Nirs signal changes during physiological challenges," PLoS Computational Biology, vol. 4, no. 11, p. e1000212, Nov 2008. [Online]. Available: http://dx.doi.org/10.1371/journal.pcbi.1000212

[23] F. F. Jöbsis, "Noninvasive, infrared monitoring of cerebral and myocardial oxygen sufficiency and circulatory parameters," Science, vol. 198, no. 4323, pp. 1264-1267, 1977. [Online]. Available: http://www.jstor.org/stable/1745848

[24] J. Steinbrink, A. Villringer, F. Kempf, D. Haux, S. Boden, and H. Obrig, "Illuminating the bold signal: combined fmri-fnirs studies," Magnetic Resonance Imaging, vol. 24, no. 4, pp. 495 - 505, 2006. [Online]. Available: http://www.sciencedirect.com/science/article/pii/S0730725X06000531

[25] M. Wolf, M. Ferrari, and V. Quaresima, "Progress of near-infrared spectroscopy and topography for brain and muscle clinical applications," Journal of biomedical optics, vol. 12, no. 6, p. 062104, 2007.

[26] M. Schecklmann, M. Romanos, F. Bretscher, M. M. Plichta, A. Warnke, and A. J. Fallgatter, "Prefrontal oxygenation during working memory in adhd," Journal of Psychiatric Research, vol. 44, no. 10, pp. 621 - 628, 2010. [Online]. Available: http://www.sciencedirect.com/science/article/pii/ S0022395609002702

[27] A. C. Rodriguez Merzagora, M. Izzetoglu, B. Onaral, and M. T. Schultheis, "Verbal working memory impairments following traumatic brain injury: an 
fnirs investigation," Brain Imaging and Behavior, vol. 8, no. 3, pp. 446-459, Sep 2014. [Online]. Available: https://doi.org/10.1007/s11682-013-9258-8

[28] F. Fishburn, M. Norr, A. Medvedev, and C. Vaidya, "Sensitivity of fnirs to cognitive state and load," Frontiers in Human Neuroscience, vol. 8, p. 76, 2014. [Online]. Available: https://www.frontiersin.org/article/10.3389/ fnhum.2014.00076

[29] H. Ayaz, P. A. Shewokis, S. Bunce, K. Izzetoglu, B. Willems, and B. Onaral, "Optical brain monitoring for operator training and mental workload assessment," NeuroImage, vol. 59, no. 1, pp. 36 - 47, 2012, neuroergonomics: The human brain in action and at work. [Online]. Available: http://www.sciencedirect.com/science/article/pii/S1053811911006410

[30] J. Harrison, K. İzzetoğlu, H. Ayaz, B. Willems, S. Hah, U. Ahlstrom, H. Woo, P. A. Shewokis, S. C. Bunce, and B. Onaral, "Cognitive workload and learning assessment during the implementation of a next-generation air traffic control technology using functional near-infrared spectroscopy," IEEE Transactions on Human-Machine Systems, vol. 44, no. 4, pp. 429-440, 2014.

[31] J. M. Baker, J. L. Bruno, A. Gundran, S. M. H. Hosseini, and A. L. Reiss, "fnirs measurement of cortical activation and functional connectivity during a visuospatial working memory task," PLOS ONE, vol. 13, no. 8, pp. 1-22, 08 2018. [Online]. Available: https://doi.org/10.1371/journal.pone.0201486

[32] H. Tsunashima and K. Yanagisawa, "Measurement of brain function of car driver using functional near-infrared spectroscopy (fnirs)," Computational intelligence and neuroscience, vol. 2009, 2009.

[33] "Optical absorption of hemoglobin." [Online]. Available: http://omlc.ogi. edu/spectra/

[34] G. S. Abdoulaev and A. H. Hielscher, "Three-dimensional optical tomography with the equation of radiative transfer," Journal of Electronic Imaging, vol. 12, no. 4, pp. 594-601, 2003. [Online]. Available: http://dx.doi.org/10.1117/1.1587730

[35] M. Schweiger, S. R. Arridge, and I. Nissilä, "Gauss-newton method for image reconstruction in diffuse optical tomography," Physics in Medicine and Biology, vol. 50, no. 10, p. 2365, 2005. [Online]. Available: http://stacks.iop.org/0031-9155/50/i=10/a =013

[36] M. J. Saikia, W. Besio, and K. Mankodiya, "Wearlight: Towards a wearable, configurable functional nir spectroscopy system for noninvasive neuroimaging," IEEE Transactions on Biomedical Circuits and Systems, pp. 1-1, 2018. 
[37] G. Schalk, D. J. McFarland, T. Hinterberger, N. Birbaumer, and J. R. Wolpaw, "Bci2000: a general-purpose brain-computer interface (bci) system," IEEE Transactions on biomedical engineering, vol. 51, no. 6, pp. 1034-1043, 2004. 


\section{MANUSCRIPT 6 \\ A machine learning approach to classify working memory load from optical neuroimaging data}

by

Manob Jyoti Saikia, Himanshu Mishra, and Kunal Mankodiya

Submitted to

IEEE INDICON 2019, Rajkot, India, Dec. 13-15, 2019 


\subsection{Abstract}

Functional near-infrared spectroscopy (fNIRS) is a brain imaging methodology which is appertaining to imaging the hemodynamic responses or blood oxygenation level-dependency of the brain. Machine learning (ML) has the potential to classify different mental states from the fNIRS signal to build a brain-computer interface (BCI) system. In this work, we have used a portable fNIRS system to image prefrontal lobe of the brain of 13 participants while they performed n-back working memory (WM) tasks. We have extracted eight important features from the hemodynamic brain signals and trained six different k-nearest neighbor $(\mathrm{k}-\mathrm{NN})$ ML classifiers. The performance of the six k-NN classifiers was evaluated with the experimental data sets. The results have shown that Weighted and Fine k-NN performed best $(95 \%)$ in classifying 5 different WM loads.

\subsection{Introduction}

Brain-computer interface (BCI) also known as mind-machine interface (MMI), is a direct communication pathway between a brain and an external device. BCI techniques are often used to research, map, assist, augment, or repair human cognitive or sensory-motor functions $[1,2]$. Several brain imaging techniques are being implemented to image the brain and to extract the important features from the brain signal for BCI purposes [3]. Among them, functional near-infrared spectroscopy (fNIRS) is a relatively new imaging modality that exhibits promising advantages for efficient BCI system $[4,5]$. fNIRS is portable compared to functional magnetic resonance imaging (fMRI) and magnetoencephalography (MEG) and has low-noise compared to electroencephalography (EEG) and MEG that are severely affected by various electrical artifacts. In addition, fNIRS is user-friendly as it does not require conductive gel during NIRS sensor placement as oppose to EEG [6]. 


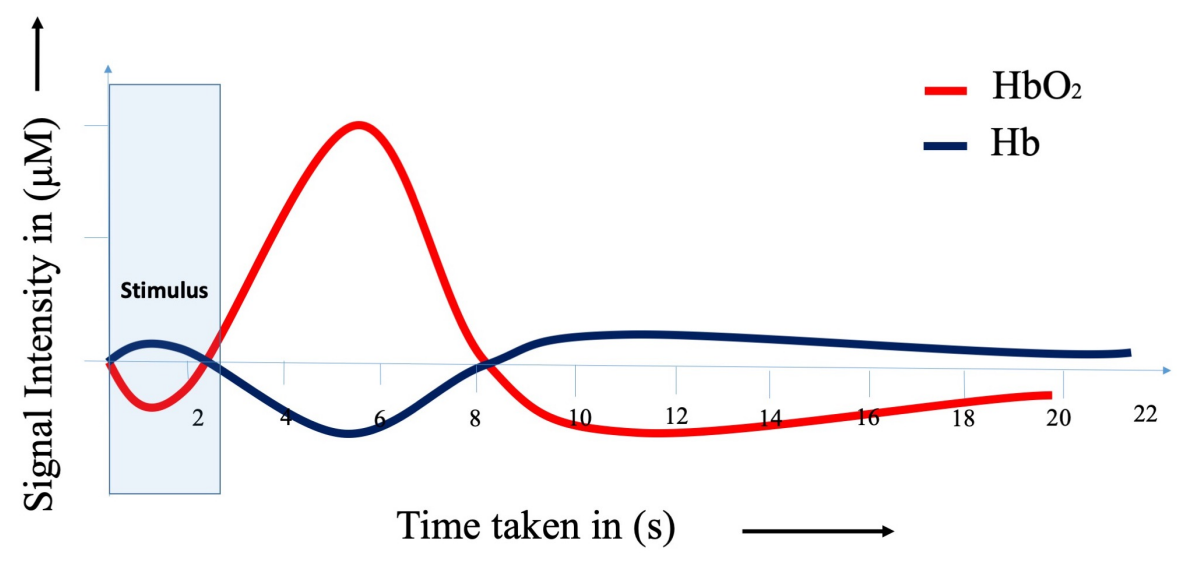

Figure 43. Typical trend of $\mathrm{HbO}_{2}$ (red curve) and $\mathrm{Hb}$ (black curve) due to cortical activation in fNIRS imaging.

The fNIRS systems use near-infrared (NIR) light sources and detectors operating in the wavelength range $600-900 \mathrm{~nm}$ [7] to measure the relative changes in oxygenated $\left(\mathrm{HbO}_{2}\right)$ and deoxygenated $(\mathrm{Hb})$ hemoglobin concentration [Fig. 43] in the cortical region of the brain $[8,6,9,10,11]$. Since hemoglobin is an oxygen carrier, changes in concentration levels of $\mathrm{HbO}_{2}$ and $\mathrm{Hb}$ during changes in brain activation levels of neuronal populations can be related to mental task-specific responses and, thus, can be used for the realization of BCI systems.

In a typical fNIRS-based BCI system graph signal processing (GSP) approach is put into work to extract all the spatial features from the signal among the timestamps and to predict brain function [5]. Investigation on various factors that affect fNIRS-based BCI, for instance, the type of features extracted during GSP, choosing the best performing algorithm for machine learning have been studied by various research groups. Also, various experimental protocols that can change the brain's hemodynamic response with respect to the tasks are used to evaluate the performance of the BCI systems.

In this paper, we used a machine learning (ML) method to classify working memory load from the fNIRS signal. We utilized a portable fNIRS system [12] to 
collect brain's hemodynamic $\left(\mathrm{HbO}_{2}\right.$ and $\left.\mathrm{Hb}\right)$ signal while participants were induced four different working memory (WM) loads using n-back WM tasks. Including four different WM loads and the rest period, we had five mental stages to be classified form the fNIRS signal. We used the k-nearest neighbor (k-NN) algorithm to model ML classifiers. From the $\mathrm{HbO}_{2}$ and $\mathrm{Hb}$ data we extracted eight important features to train six different k-NN classifiers. The accuracy in classifying the five mental states of the participants was evaluated.

\subsection{Background of fNIRS-based BCI}

The development of optical methods for brain imaging originated from the muscle oximeter invented by Glenn Millikan in the forties [13]. It is well known that brain activity is associated with a number of physiological events; some of which are associated with changes in the optical properties of brain tissue, and can be assessed by optical techniques. fNIRS uses the principle of change in intensity of NIR light when passed through the brain due to change in the concentration of blood oxygen in the brain $[8,6,14,15]$. It offers to monitor the real-time changes in concentration of oxygenated $\left(\mathrm{HbO}_{2}\right)$ and deoxygenated $(\mathrm{Hb})$ hemoglobin as well as the changes in the redox state of cytochrome-c-oxidase (Cyt-Ox) by their different specific spectra in the NIR range.

Machine learning (ML) is a method that uses algorithms and statistical models implemented in the computer system in order to perform a specific task effectively without using explicit instructions, relying on patterns and inference instead. ML algorithm builds a mathematical model based on sample data, known as "training data", in order to make predictions or decisions without being explicitly programmed to perform the task.

Primarily the algorithms implemented for ML in fNIRS by researchers utilize the support vector machine (SVM) model. SVM performs better when the number 
of data is limited $[16,17]$. Few researchers employed linear discriminant analysis (LDA) to classify the signals for EEG and fNIRS, and they found it to be faster than SVM [18]. It utilizes discriminant hyperplane(s) to separate data representing two or more classes. Because of its simplicity and low computational requirements, LDA is highly suitable for online BCI systems. Many fNIRS and hybrid EEGfNIRS studies have successfully used LDA for BCIs.

LDA is also closely related to principal component analysis (PCA) and factor analysis in that they look for linear combinations of variables which best explain the data. Since it's more closed to PCA it requires a lot more computational attention. While on the other hand, the k-nearest neighbors (k-NN) algorithm typically uses mathematical equations such as Euclidean distances between two points and other not much complex functions to compute easily. And then categorize them on the basis of the values yielded by these functions. Hence, implementation is very efficient in terms of time taken to train and deliver accurate results in classifying.

\subsection{Materials and methods \\ 6.4.1 Experimental study}

We have used a portable fNIRS system [12] to collect hemodynamic activities from the prefrontal lobe of the brain. The participants performed a computergenerated n-back working memory (WM) task [19]. We requited 13 participants in

our experimental study. In the n-back task, WM load increases with the increasing number of items (n) to be memorized. In the experimental protocol, we had sixteen n-back task blocks, consisting of 0,1,2 and 3-back task conditions as shown in the figure 44 (a). The order of these four conditions was pseudo-randomized and duration of the data collection was about 12 minutes for each participant. The raw fNIRS signal from the prefrontal lobe of the brain [Fig. 44(b)] was processed and converted to hemodynamic $\left(\mathrm{HbO}_{2}\right.$ and $\left.\mathrm{Hb}\right)$ response as explained in our previous 
study $[12]$.

(a)

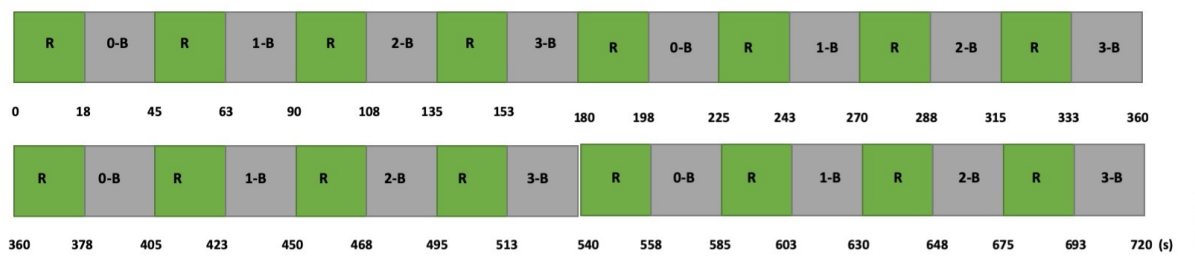

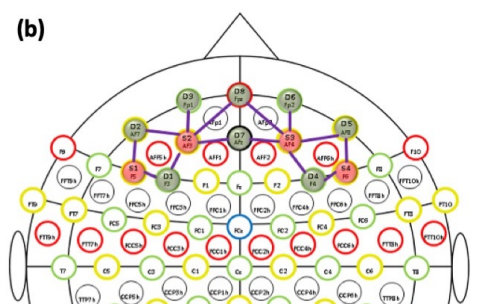

(b)

Figure 44. (a) Experimental protocol with four n-back conditions (0-back, 1-back, 2-back and 3-back) and rest $(\mathrm{R})$ periods. The 16 random task blocks in one run takes about 720 seconds. (b) fNIRS light source (red) and detector (green) locations on the prefrontal lobe of the brain.

\subsubsection{Machine learning implementation}

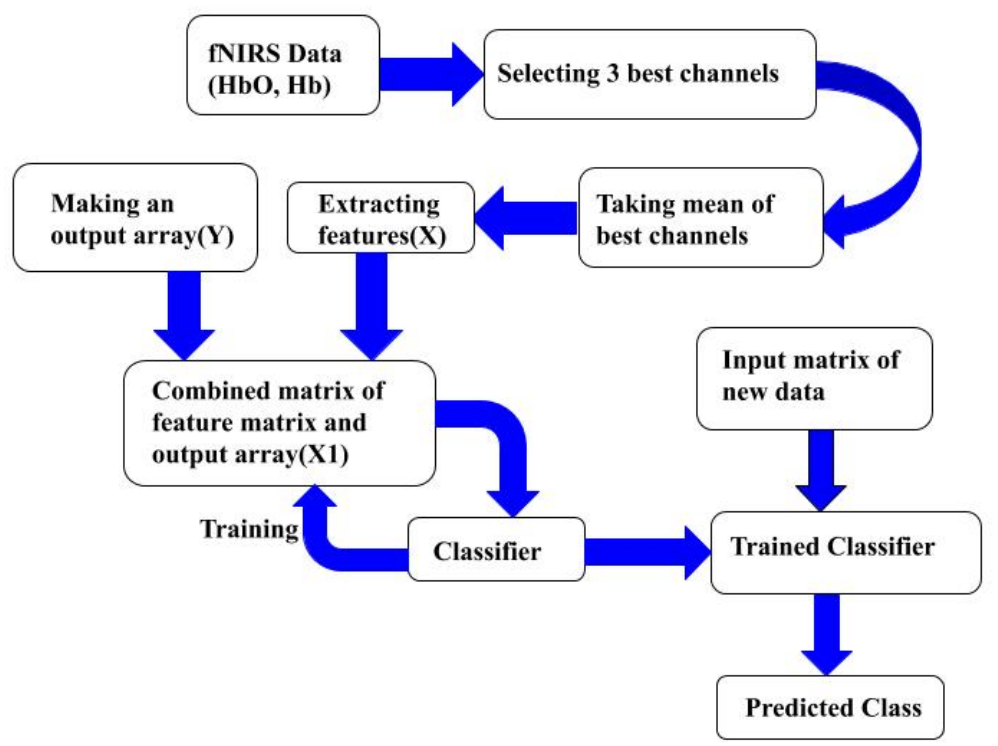

Figure 45. Flow chart of our ML implementation. The extracted feature matrix (X) from the fNIRS signal (mean of best three channels) was combined with output matrix (Y) to form X1 matrix to train the classifier. The trained classifier was tested with input data sets to predict mental states (5 classes).

As shown in the flowchart in Fig. 45, best 3 fNIRS channels were identified after performing baseline correction method [18] on all the fNIRS channels. In the baseline correction process, we compared the maximum hemodynamic response 
during the task period (n-back task) and the rest period. We considered an fNIRS channel as active and processed further if the difference was positive. We found that this method was effective as it required the least computational expenses with the ability to rank all the fNIRS channels. The first three channels that showed the maximum changes in hemodynamic response were selected and then they were averaged to get the mean signal. Our goal is to classify different nback tasks over time from the fNIRS signal to classify different WM load levels of the participants. Hence, comparing and averaging the fNIRS channels performed better in our application as it reduced the data redundancy and minimized the size of the feature matrix $(\mathrm{X})$ for the computational efficiency.

\section{Feature extraction}

Figure 43 presents the trend of a typical fNIRS signal where $\mathrm{HbO}_{2}$ increases and $\mathrm{Hb}$ decreases slightly due to the brain stimulation. Various important features from the $\mathrm{HbO}_{2}$ and $\mathrm{Hb}$ for the BCI purposes are suggested [20, 21, 22, 23, 24]. In our study, we have extracted eight important features from the mean fNIRS signal as seen in the flowchart in Fig. 45. They are (1) Gradient value, (2) Mean, (3) Variance, (4) No. of peaks, (5) Kurtosis, (6) Skewness, (7) Max and (8) Min value. These are the most widely used features in fNIRS and EEG studies.

We created a feature matrix $(\mathrm{X})$ from the data set and combined the corresponding output matrix $(\mathrm{Y})$ to form a matrix (X1) as shown in the figure 45 . The Y matrix stored the actual output corresponding to the feature matrix. X1 was passed onto the classifier to train itself. Once we get the trained classifier we used it to predict the 4 levels of WM load and rest period from the fNIRS input data sets we obtained from the n-back task experimentation. We had 4 classes corresponding to each n-back task and rest region was treated as a separate class. Therefore, in total, we had 5 classes to predict. 


\section{Classification using K-nearest neighbor}

We used the k-nearest neighbor (k-NN) algorithm for our machine learning approach. This algorithm works by using an input vector with the k closest training samples in the feature space. To perform the classification, the algorithm identifies the most common class among the $\mathrm{k}$ nearest neighbors. The algorithm requires training to define the neighbors based on the distance from the test sample and a testing step to determine the class to which this test sample belongs. The number of neighbors can be changed to adjust the k-NN algorithm. In this sense, for instance, the use of one neighbor is known as fine k-NN, and a coarse k-NN uses 100 neighbors. Many neighbors can be time consuming to fit. We used six different k-NN classifiers to classify data, and these classifiers are based on different distances. For training the classifier we used MatLab's classification learner App. The six classifiers implemented are Fine k-NN, Medium k-NN, Coarse k-NN, Cosine k-NN, Cubic k-NN, and Weighted k-NN.

After training and obtaining the six classifiers, we evaluated the performance of these trained classifiers with the input data sets. Input $\mathrm{HbO}_{2}$ and $\mathrm{Hb}$ datasets are transformed into the feature space before testing the different classifiers.

Table 4. Trained six k-NN classifiers and corresponding accuracy in classification

\begin{tabular}{|c|c|}
\hline Classifier name & Accuracy (\%) \\
\hline Fine k-NN & 95 \\
\hline Medium k-NN & 93 \\
\hline Coarse k-NN & 89 \\
\hline Cosine k-NN & 93 \\
\hline Cubic k-NN & 93 \\
\hline Weighted k-NN & 95 \\
\hline
\end{tabular}




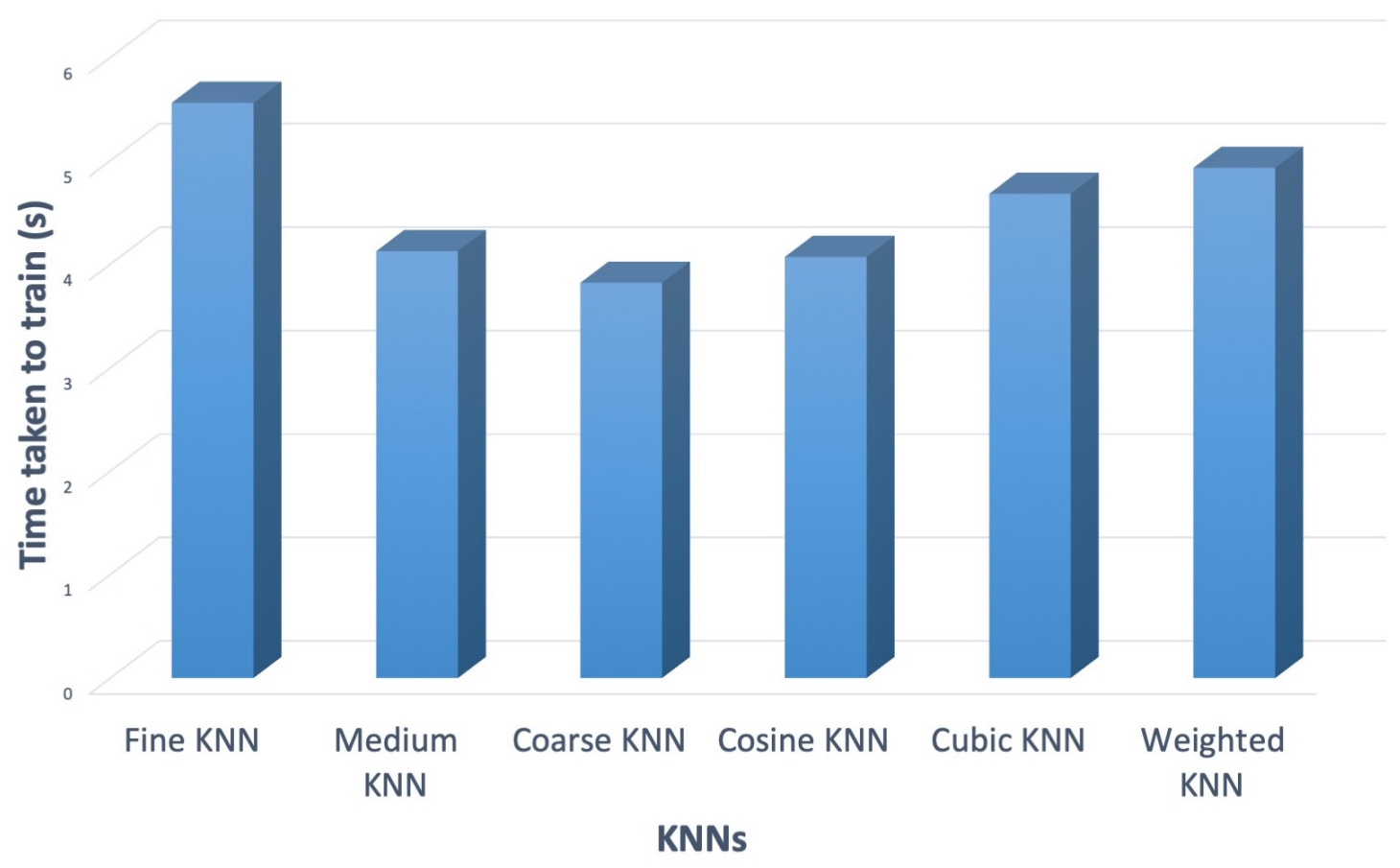

Figure 46. Computation time in training the six k-NN classifiers.

\subsection{Results and Discussion}

As shown in Table 1 Weighted and Fine k-NN delivered the most concurred and superior results as they performed the best among all the k-NN classifier tested, with the accuracy of $95 \%$ each. Medium, Cosine and Cubic k-NN also performed well with the accuracy of $93 \%$ each. The results have been remarkable, and the notable point is that the data for training needs to be collected one time only. Hence, once the classifier is trained it is possible to use it in real-time to predict the task that's being performed or to predict WM load.

While training the different k-NN classifiers with training data sets in the form of a combined matrix (X1) as shown in Fig. 45, we discovered that Fine k-NN took the longest and Coarse k-NN took the least time to train [Fig. 46]. It was expected since in Fine k-NN we had $\mathrm{k}$ being equal to 1 . Therefore, it made a much larger number of batches as compared to coarse $\mathrm{k}-\mathrm{NN}$ where $\mathrm{k}$ is equal to 100. Moreover, Fine k-NN had smaller area per batch as compared to Coarse 
k-NN. So, it was relatively more computationally expensive. And there were much better chances that it will overfit as compared to Coarse k-NN.

\subsection{Conclusion}

The accuracy of each trained classifier in classifying the 5 different levels of working memory loads was evaluated and found that Fine and Weighted k-NN performed best with an accuracy of $95 \%$. The preliminary results in terms of classification accuracy are promising and it encourages us to perform more experimental studies in the future. We shall also train and evaluate the performance of the k-NN classifiers and others using broad data sets in the future. Also, the real-time classification of the mental workload using a portable fNIRS system augmented by advanced machine learning techniques can be an interesting application, hence, we shall perform extensive studies on it in the future.

\section{Acknowledgment}

This material is based upon work supported by the National Science Foundation under Grant No. 1539068. The authors would also like to thank Joanne Hall, Lynn McCane and Shunan Li for their assistance.

\section{List of References}

[1] U. Chaudhary, N. Birbaumer, and A. Ramos-Murguialday, "Braincomputer interfaces for communication and rehabilitation," Nature Reviews Neurology, vol. 12, pp. 513 EP -, 08 2016. [Online]. Available: http://dx.doi.org/10.1038/nrneurol.2016.113

[2] M. O. Krucoff, S. Rahimpour, M. W. Slutzky, V. R. Edgerton, and D. A. Turner, "Enhancing nervous system recovery through neurobiologics, neural interface training, and neurorehabilitation," Frontiers in neuroscience, vol. 10, pp. 584-584, 12 2016. [Online]. Available: https://www.ncbi.nlm.nih. gov/pubmed/28082858

[3] N. Naseer and K.-S. Hong, "fnirs-based brain-computer interfaces: a review," Frontiers in Human Neuroscience, vol. 9, p. 3, 2015. [Online]. Available: http://journal.frontiersin.org/article/10.3389/fnhum.2015.00003 
[4] S. M. Coyle, T. E. Ward, and C. M. Markham, "Brain-computer interface using a simplified functional near-infrared spectroscopy system," Journal of Neural Engineering, vol. 4, no. 3, p. 219, 2007. [Online]. Available: http://stacks.iop.org/1741-2552/4/i=3/a=007

[5] P. C. Petrantonakis and I. Kompatsiaris, "Single-trial nirs data classification for brain-computer interfaces using graph signal processing," IEEE Transactions on Neural Systems and Rehabilitation Engineering, vol. 26, no. 9, pp. 1700-1709, 2018.

[6] M. Ferrari and V. Quaresima, "A brief review on the history of human functional near-infrared spectroscopy (fnirs) development and fields of application," NeuroImage, vol. 63, no. 2, pp. 921 - 935, 2012. [Online]. Available: http://www.sciencedirect.com/science/article/pii/ S1053811912003308

[7] K. M. Manob Jyoti Saikia, "3d-printed human-centered design of fnirs optode for the portable neuroimaging," 2019. [Online]. Available: https://doi.org/10.1117/12.2510955

[8] F. F. Jöbsis, "Noninvasive, infrared monitoring of cerebral and myocardial oxygen sufficiency and circulatory parameters," Science, vol. 198, no. 4323, pp. 1264-1267, 1977. [Online]. Available: http://www.jstor.org/stable/1745848

[9] R. K. Manob Jyoti Saikia, "A tabletop diffuse optical tomographic (dot) experimental demonstration system," 2019. [Online]. Available: https://doi.org/10.1117/12.2510857

[10] F. Irani, S. M. Platek, S. Bunce, A. C. Ruocco, and D. Chute, "Functional near infrared spectroscopy (fnirs): An emerging neuroimaging technology with important applications for the study of brain disorders," The Clinical Neuropsychologist, vol. 21, no. 1, pp. 9-37, 2007, pMID: 17366276. [Online]. Available: https://doi.org/10.1080/13854040600910018

[11] M. J. Saikia and R. Kanhirodan, "Development of handheld near-infrared spectroscopic medical imaging system," in Biophotonics Congress: Optics in the Life Sciences Congress 2019 (BODA,BRAIN,NTM,OMA,OMP). Optical Society of America, 2019, p. DS1A.6. [Online]. Available: http://www.osapublishing.org/abstract.cfm?URI=BODA-2019-DS1A.6

[12] M. J. Saikia, W. G. Besio, and K. Mankodiya, "Wearlight: Toward a wearable, configurable functional nir spectroscopy system for noninvasive neuroimaging," IEEE Transactions on Biomedical Circuits and Systems, vol. 13, no. 1, pp. 91-102, 2019.

[13] G. A. Millikan, "The oximeter, an instrument for measuring continuously the oxygen saturation of arterial blood in man," Review of Scientific 
Instruments, vol. 13, no. 10, pp. 434-444, 1942. [Online]. Available: https://doi.org/10.1063/1.1769941

[14] M. Abtahi, G. Cay, M. J. Saikia, and K. Mankodiya, "Designing and testing a wearable, wireless fnirs patch," in 2016 38th Annual International Conference of the IEEE Engineering in Medicine and Biology Society (EMBC), Aug 2016, pp. 6298-6301.

[15] R. K. Manob Jyoti Saikia, Kunal Mankodiya, "A point-of-care handheld region-of-interest (roi) 3d functional diffuse optical tomography (fdot) system," 2019. [Online]. Available: https://doi.org/10.1117/12.2510926

[16] R. A. Shirvan, S. K. Setaredan, and A. M. Nasrabadi, "Classification of mental stress levels by analyzing fnirs signal using linear and non-linear features," International Clinical Neuroscience Journal, vol. 5, no. 2, pp. 55-61, 2018.

[17] M. Abtahi, Y. Shahriari, M. J. Saikia, G. Cay, U. Akbar, and K. Mankodiya, "Eeg-fnirs combined neuroimaging study on pd patients performing updrs motor tasks (p5.8-020)," Neurology, vol. 92, no. 15 Supplement, 2019. [Online]. Available: https://n.neurology.org/content/92/15_Supplement/P5.8-020

[18] K.-S. Hong, M. J. Khan, and M. J. Hong, "Feature extraction and classification methods for hybrid fnirs-eeg brain-computer interfaces," Frontiers in Human Neuroscience, vol. 12, p. 246, 2018. [Online]. Available: https://www.frontiersin.org/article/10.3389/fnhum.2018.00246

[19] W. K. Kirchner, "Age differences in short-term retention of rapidly changing information." Journal of Experimental Psychology, vol. 55, no. 4, pp. 352-358, 1958.

[20] H.-J. Hwang, H. Choi, J.-Y. Kim, W.-D. Chang, D.-W. Kim, K. Kim, S. Jo, and C.-H. Im, "Toward more intuitive brain-computer interfacing: classification of binary covert intentions using functional near-infrared spectroscopy," Journal of biomedical optics, vol. 21, no. 9, p. 091303, 2016.

[21] F. M. Noori, N. Naseer, N. K. Qureshi, H. Nazeer, and R. A. Khan, "Optimal feature selection from fnirs signals using genetic algorithms for bci," Neuroscience Letters, vol. 647, pp. 61-66, 2017. [Online]. Available: http://www.sciencedirect.com/science/article/pii/S0304394017302239

[22] L. Holper and M. Wolf, "Single-trial classification of motor imagery differing in task complexity: a functional near-infrared spectroscopy study," Journal of NeuroEngineering and Rehabilitation, vol. 8, no. 1, p. 34, 2011. [Online]. Available: https://doi.org/10.1186/1743-0003-8-34

[23] M. J. Khan and K.-S. Hong, "Passive bci based on drowsiness detection: an fnirs study," Biomedical optics express, vol. 6, no. 10, pp. 4063-4078, 09 2015. [Online]. Available: https://www.ncbi.nlm.nih.gov/pubmed/26504654 
[24] M. Stangl, G. Bauernfeind, J. Kurzmann, R. Scherer, and C. Neuper, "A haemodynamic brain-computer interface based on real-time classification of near infrared spectroscopy signals during motor imagery and mental arithmetic," Journal of Near Infrared Spectroscopy, vol. 21, no. 3, pp. 157-171, 2019/07/16 2013. [Online]. Available: https://doi.org/10.1255/jnirs.1048 